SCHOOL OF CIVIL ENGINEERING

JOINT HIGHWAY

RESEARCH PROJECT

FHWA/IN/JHRP-81/15

PORE SIZE DISTRIBUTION OF SANDY SOILS AND THE PREDICTION OF PERMEABILITY

C.H. Juang 
Digitized by the Internet Archive in 2011 with funding from

LYRASIS members and Sloan Foundation; Indiana Department of Transportation 


\section{Interim Report \\ PORE SIZE DISTRIBUTION OF SANDY SOILS \\ AND \\ THE PREDICTION OF PERMEABILITY}

To: II. L. Michae1, Director

Joint Highway Research Project

From: R. D. Holtz, Research Engineer Joint Highway Research Project
August 13, 1981

Project: $\quad C-36-5 N$

File: 6-6-14

Attached is an Interim Report on the HPR-1(19) Part II Research Study entitled "Effects of Pore Size Distribution on Permeability and Frost Susceptibility of Selected Subgrade Materials". This is the fourth report from this study and it covers Task $\mathrm{E}$ of the approved work plan. The author of the report is $\mathrm{Mr}$. Charng-Hsein Juang who worked under the supervision of Prof. C. W. Lovell and myself. The report title is "Pore Size Distribution of Sandy Soils and the Prediction of Permeability".

The results of the study show the usefulness of pore size distribution parameters for characterizing the fabric of sandy soils. They are also useful for predicting the permeability of such soils. The author has developed a statistical prediction equation which uses the PSD parameters and which is shown to give an accurate estimate of permeability for compacted soils ranging from sands to clays.

The Report is a partial fulfillment of the objectives of the Study. Copies will be submitted to the IDOH and FHWA for their review, comment, and acceptance.

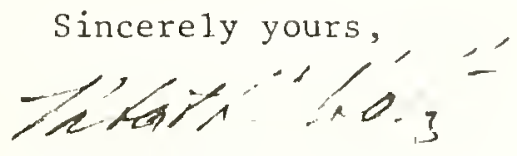

R. D. Holtz

Research Engineer

$\mathrm{RDH}: \mathrm{cm}$

cc: A. G. Altschaeffl
W. L. Dolch
R. L. Eskew
G. D. Gibson
W. H. Goetz
M. J. Gutzwiller
G. K. Hallock

D. E. Hancher

J F. McLaughlin

R. D. Miles

P. L. Owens

G. T. Satterly
C. F. Scholer

K. C. Sinha

C. A. Venable

L. E. Wood

E. J. Yoder

S. R. Yoder 
PORE SIZE DISTRIBUTION OF SANDY SOILS

AND

THE PREDICTION OF PERMEABILITY

\author{
by \\ Charng-lisein Juang \\ Graduate Instructor in Research \\ Joint Highway Research Project \\ Project No.: $\mathrm{C}-36-5 \mathrm{~N}$ \\ File No.: 6-6-14 \\ Prepared as Part of an Investigation \\ Conducted by \\ Joint Highway Research Project \\ Engineering Experiment Station \\ Purdue University \\ in cooperation with the \\ Indiana Department of Highways \\ and the \\ U.S. Department of Transportation \\ Federal Highway Administration
}

The contents of this report reflect the views of the author who is responsible for the facts and the accuracy of the data presented herein. The contents do not necessarily reflect the official views or policies of the Federal Highway Administration. The report does not constitute a standard, specification, or regulation. 
TECHNICAL REPORT STANDARD TITLE PAGE

\begin{tabular}{|c|c|}
\hline $\begin{array}{l}\text { 1. Roport No. } \\
\text { FHWA / IN/JHRP-81/15 }\end{array}$ & 3. Rocipiont's Co:olog No. \\
\hline \multirow{2}{*}{$\begin{array}{l}\text { 4. Tirle and Subrile } \\
\text { PORE SIZE DISTRIBUTION OF SANDY SOILS AND } \\
\text { THE PREDICTION OF PERMEABILITY }\end{array}$} & $\begin{array}{l}\text { 5. Roport Doto } \\
\text { August 13, } 1981\end{array}$ \\
\hline & 6. Perfarming Orgonization Code \\
\hline $\begin{array}{l}\text { 7. Author(s) } \\
\text { Charng-Hse in Juang } \\
\end{array}$ & $\begin{array}{l}\text { 8. Parforming Organizalion Roport No. } \\
\text { JHRP-8I-15 }\end{array}$ \\
\hline \multirow{3}{*}{$\begin{array}{l}\text { 9. Perfarming Organixotion Namo and Addross } \\
\text { Joint Highway Research Project } \\
\text { Civil Engineering Building } \\
\text { Purdue University } \\
\text { W. Lafayette, Indiana } 47907\end{array}$} & 10. Work Unif No. \\
\hline & $\begin{array}{l}\text { 11. Contract ar Grant No. } \\
\text { HPR-1( 19) Part II }\end{array}$ \\
\hline & 13. Typo of Repore and Period Covered \\
\hline \multirow{2}{*}{$\begin{array}{l}\text { 12. Sponsoring Agoncy Nome ond Addres: } \\
\text { Indiana Department of Highways } \\
\text { State Office Building } \\
100 \text { North Senate Avenue } \\
\text { Indianapolis, Indiana } 46204\end{array}$} & Interim Report \\
\hline & 14. Sponsoring Agency Codo \\
\hline
\end{tabular}

15. Supplementory Notos

Prepared in cooperation with the U.S. Department of Transportation, Federal

Highway Administration. Part of the Study titled "Effects of Pore Size Distribution on Permeability and Frost Susceptibility of Selected Subgrade Materials". 16. Abstroct

The soils used in this study were a sand and two sand-clay mixtures. Kneading compaction was used to prepare samples of sand-clay mixtures while pluvial compaction was used to prepare compacted samples of sand. The testing program consisted of the permeability test and the pore size distribution (PSD) test. A technique for the preparation of PSD specimens of cohesionless sands has been developed. The mercury intrusion technique was used to conduct PSD test. Falling head tests under back pressure were used to measure permeability.

The pore size density function of the sands studied showed a single modal characteristic on a log diameter scale while that of sand-clay mixtures showed bimodal characteristics on the same scale. The influence of varying water content and compactive effort on the fabric of the sandy soils studied was characterized by their pore size distributions and pore size density functions.

A permeability model which related the pore size distribution to the permeability of soils has been proposed. The prediction of permeability by the proposed model was excellent $\left(\mathrm{e} \cdot \mathrm{g} \cdot \mathrm{R}_{\mathrm{a}}^{2}=0.98\right.$ ) for both the author's and Garcia-Bengochea's (1978) data. Thus, the proposed permeability model can provide an excellent predictive tool for a rather wide range of compacted soils.

17. Koy Words

compacted sandy soils, pore size distribution, permeability
18. Distribution Statement

No restrictions. This document is available to the public through the National Technical Information Service, Springfield, Virginia 22161.
19. Securlty Clasaif. (of this roport) Unclass ified
20. Securlity Clossif. (of this pope)

Unclassified
22. Price

\section{Form DOT F 1700.7 (8.69)}




\section{ACKNOWLEDGEMENTS}

The author wishes to express his sincere gratitude to Professors R. D. Holtz and C. W. Lovell, his major advisor and former advisor respectively, for the contributions of their time, effort, and ideas to the work presented in this dissertation. Special thanks are also due Professor Lovell for helping the author to surmount personal setbacks and obstacles.

The author is also greatly indebted to Professor D. N. Winslow for valuable discussions on mercury intrusion technology, and to Professor G. P. McCabe for helpful discussions on mathematical problems.

Special thanks are due Professor M. E. Harr for his support during the author's first-year study at Purdue.

The laboratory assistance provided by Mrs. Janet Lovell was extremely helpful. The secretarial work by Mrs. Edith Vanderwerp and thesis typing by Ms. Linda Gibson were greatly appreciated.

The financial support for this research was provided by the State of Indiana and the Federal Highway Administration through the Joint Highway Research Project at Purdue University.

Finally, very special thanks are extended to my wife, Anne, for her assistance in both laboratory and computer work, and many sacrifices during the course of this study; indeed this dissertation would not have been possible without her help. 
TABLE OF CONTENTS

Page

LIST OF TABLES

$v i$

LIST OF FIGURES. . . . . . . . . . . . . . . . . . . . vi1

LIST OF ABBREVIATIONS . . . . . . . . . . . . . . . . .

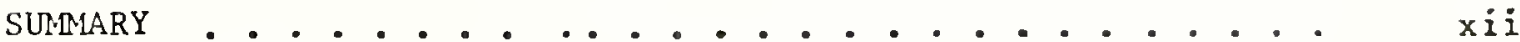

CHAPTER I INTRODUCTION. . . . . . . . . . . . . . . . 1

1.1 Fabric and Its Role in Geotechnical Engineering. . . 1

1.2 Objective of This study ............. 3

CHAPTER II THEORETICAL BACKGROUND. . . . . . . . . . . . 4

2.1 Pore Size Distribution ............ 4

2.1.1 Theoretical Background of Pore Size

Distribution and Pore Size Density Function.

4

2.1.2 The Measurement of Pore Size Distribution

and Its Limitation............ 9

2.1.3 The Applications of Pore Size Distribution. . II

2.2 Permeability Model .............. . . . 12

2.2.1 Relation of Permeability to Pore Geometry . . 12

2.2.2 Proposed Permeability Model . . . . . . 16

CHAPTER III TESTING PROGRAM AND EXPERIMENTAL PROCEDURES . • . 23

3.1 Testing Program. . . . . . . . . . . . . 23

3.2 Soll Studied . . . . . . . . . . . . . . . 25

3.3 Compaction . . . . . . . . . . . . . . 25

3.3.1 Pluvial Compaction for Soll A........ 25

3.3.2 Kneading Compaction for Solls B and C . . . 28 
Page

3.4 Permeability Tests................ . . 31

3.5 Specimen Preparation and Drying and Pore Size

Distribution Tests. . . . . . . . . . . . .

CHAPTER IV PRESENTATION AND DISCUSSION. . . . . . . . . .

4.1 Compaction. . . . . . . . . . . . . . 39

4.1.1 Sample Nomenclature. . . . . . . . . 39

4.1.2 Dry Density versus Water Content

Relationships for Soils B and C. . . . . .

4.1.3 Pluvial Compaction Result for Soil A . . . .

4.2 Permeability. . . . . . . . . . . . 4 45

4.3 Pore Size Distribution. . . . . . . . . . . 50

4.3.I Data Reduction.............. 50

4.3.2 Data Presentation. . . . . . . . . . 52

4.3.3 Pore Size Distribution Curves and
Fittings of the Curves . . . . . . . . .

4.3.4 Comparison of Pore Size Curves........ 71

4.4 Prediction of Permeability by Proposed Model. . . . . 85

4.5 Applicability of Proposed Permeability Model. . . . 91

CHAPTER V SUMMARY, CONCLUSIONS AND RECOMMENDATIONS . . . . . 96

5.1 Summary .. . . . . . . . . . . . . . 9 96

5.2 Conclusions . . . . . . . . . . . . . . . 9 98

5.3 Recommendations for Future Research ......... 101

REFERENCES . . . ........................ 103

APPENDICES

Appendix A-1: Freeze Dry1ng Method .......... 108

Appendix A-2: Determination of Pore Size Distribution. . 112

Appendix A-3: Computer Program for PSD Data

Reduction and plotting and

Sample Output. . . . . . . . . . 
Appendix A-4: The Graphical Presentations of

PSD Original Data. . . . . . . . . . .

Appendix A-5: Use of the Computer Program

SPLINE and Results . . . . . . . . . .

Appendix A-6: Calculations of Functional

Values from SPLINE . . . . . . . . . .

Appendix A-7: Correction of Unconnected Pores. . . . .

Appendix A-8: Pore Size Curves Expressed as a

Percentage of Total Pore Volume. . . . . .

Appendix A-9: Prediction of Permeability: Computer

Program and Output . . . . . . . .

Appendix A-10: Prediction of Permeability :

Garcia-Bengochea's (1978) Data . . . . 


\section{LIST OF TABLES}

Table

Page

3-1 Classification Properties of Soils Studied . . . . .

4-1 Sample Code Designation. . . . . . . . . . . 40

4-2 Specifications for Impact Compaction Test. . . . . . .

4-3 Impact Compaction Data and Coefficient of Multiple Deternination . . . . . . . . . . .

4-4 Kneading Compaction Variables for Each

Sample Point of Soils B and C. . . . . . . . .

4-5 Pluvial Compaction Variables for Each Sample Point of Soil A. . . . . . . . . . . . . 46

4-6 Permeabilities of Samples Tested . . . . . . . . .

4-7 Predictions of Permeabilities by Gross

Volumetric Parameters. . . . . . . . . . .

4-8 The v - Values ..................

4-9 Possible Correlations between PSD Parameters and $v$. .

4-10 Comparison of Predicted and Measured Permeability. . . 


\section{LIST OF FIGURES}

Figure

Page

1-1 Schematic Diagram of Sand Fabric (After Leonards, as cited by Holtz and Kovacs, 1981). . . . . . .

2-1 Schematic Representation of a Sectioned Soil

(After Hillel, 1980) ...............

2-2 Schematic Representation of a Flow Region. . . . . .

2-3 Family of Governing Functions. . . . . . . . . . .

3-1 Flow Chart of the Testing Program. . . . . . . .

3-2 Grain Size Distribution of Soils Studied . ......

3-3 Schematic Diagram of Pluviation. . . . . . . . . .

3-4 Kneading Compactor . . . . . . . . . . . . . .

3-5 Compaction Curves for $90 \%$ Silt and $10 \%$ Kaolin (After Garcia-Bengochea, 1978)

3-6 Saturation and Permeation Device . . . . . . . . .

4-1 Dry Density versus Water Content Curves

For Soils B and C. . . . . . . . . . . . . .

4-2 Schematic Diagram of Falling Head Permeability

Test (After Garcia-Bengochea, 1978). . . . . . . .

4-3 (a) Frequency Histogram. . . . . . . . . . . . .

4-3(b) Finite Difference Approximation to PSD

Density Function . . . . . . . . . . . . . .

4-4 Comparison of Finite Difference Approximation

Method and Direct Differenttation. . . . . . . . .

4-5 Flow Chart of Procedures for the PSD curves......

4-6 PSD Curves of SC1MW. . . . . . . . . . . . . .

4-7 PSD Curves of SClMO. . . . . . . . . . . . . . 
4-9 PSD Curves of SC3MW. ................... 63

4-10 PSD Curves of SC3MO. . . . . . . . . . . . 64

4-11 PSD Curves of SC3MD. . . . . . . . . . . 65

4-12 PSD Curves of SC3HO-D. . . . . . . . . . . . 66

4-13 PSD Curves of SC3HD. . . . . . . . . . . . 67

4-14 PSD Curves of OPL. . . . . . . . . . . . . 68

4-15 PSD Curves of $\mathrm{OPH}$. . . . . . . . . . . 69

4-16 PSD Curves of SC3MD (Percent of Tota1

Pore Volume Existing). . . . . . . . . . . . . .

4-17 PSD Curves of SC3MD (Percent of Total

Pore Volume Intruded). . . . . . . . . . . . 73

4-18 Comparison of PSD Curves for Samples Compacted at Different Water Contents (SC3M series). . . . . .

4-19 Comparison of PSD Curves for Samples Compacted at Different Water Contents (SC3H series). . . . . .

4-20 Comparison of PSD Curves for Samples Compacted at Different Water Contents (SC1M series). . . . . .

4-21 Comparison of PSD Curves for Samples Compacted at Different Compactive Efforts (Soil C) . . . . . .

4-22 Comparison of PSD Curves for Samples Compacted at Different Compactive Efforts (Soil A) . . . . .

4-23 Comparison of PSD Curves for Different Soils (B and C) Compacted by Medium Effort at Dry of Optimum Water

Content.

4-24 Comparison of PSD Curves for Different Soils ( $B$ and C) Compacted by Medium Effort at Optimum Water Content. .

4-25 Comparison of PSD Curves for Different Soils (B and C) Compacted by Medium Effort at Wet of Optimum Water

4-26 Comparison of PSD Curves for Different Soils (A, B and C)................. .

4-27 Prediction of Permeability by Proposed Model . . . . . 
Figure

4-28 Prediction of Prermeability by Proposed Model

(Author's and Ciarcia-Bengochra's, 1978 nata) . . . .

Appendix

Figure

A-1-1 Sample Cage Suspended in Desjccator

(From Reed, 1977). . . . . . . . . . . . . .

A-1-2 Freeze Drying Apparatus with Paclum Punp,

Condenser ind Desiccator (From Feed, 1977). . . .

A-2-1 Penetrometers, Plastic Cap, Hetal Cap

and Vial with Specimens. . . . . . . . . . . .

A-2-2 Filling Device and Hcieod Gauge. . . . . . . . .

A-2-3 Mercury Manometer and Bleeder Valve. . . . . . . .

A-2-4 Control System for Low Pressure Intrusion (After White, 1980).................. 116

A-2-5 Aninco Porosimeter . . . . . . . . . . . . . 117

A-4-1 PSD Curves of SCIM (From Original Data) . . . . . 136

A-4-2 PSD Curves of SCIMO (From Original Data) . . . . . 137

A-4-3 PSD Curves of SCIMD (Frotn Original Data) ....... 138

A-4-4 PSD Curves of SC3IJ (From Original Data) . ...... 139

A-4-5 PSD Curves of SC3MO (From Original Data) . . . . . 140

A-4-6 PSD Curves of SC3MD (From Original Data) . . . . . 141

A-4-7 PSD Curves of SC3HO-D (From Original Data) • . . . . 142

A-4-8 PSD Curves of SC3HD (From Original Data) . . . . . . 143

A-4-9 PSD Curves of OPL (From Original Data) . . . . . . . 144

A-4-10 PSD Curves of OPH (From Original Data) . . . . . . 148

A-8-1 PSD Curves of SCIM (Percent of Total Pore

Volume Existing) . . . . . . . . . . . . 174

A-8-2 PSD Curves of SCIIN (Percent of Total Pore

Volume Intruded) . . . . . . . . . . . . .

A-8-3 PSD Curves of SCIMO (Percent of Total Pore

Volume Existing) . . . . . . . . . . . . . 
Appendix

Figure

A-8-4 PSD Curves of SC1MO (Percent of Total Pore

A-8-5 PSD Curves of SCIMD (Percent of Total Pore Volume Existing) . . . . . . . . . . . . .

A-8-6 PSD Curves of SCIMD (Percent of Total Pore Volume Intruded) . . . . . . . . . . . . . .

A-8-7 PSD Curves of SC3MN (Percent of Total Pore Volume Existing)

A-8-8 PSD Curves of SC3MN (Percent of Total Pore Volume Intruded) . . . . . . . . . . . . . .

A-8-9 PSD Curves of SC3MO (Percent of Total Pore Volume Existing) . . . . . . . . . . . . .

A-8-10 PSD Curves of SC3MO (Percent of Total Pore Volume Intruded) . . . . . . . . . . . . . .

A-8-11 PSD Curves of SC3MD (Percent of Total Pore Volume Existing) . . . . . . . . . . . . .

A-8-12 PSD Curves of SC3MD (Percent of Total Pore Volume Existing) . . . . . . . . . . . . . .

A-8-13 PSD Curves of SC3HO-D (Percent of Total Pore Volume Existing) . . . . . . . . . . . . .

A-8-14 PSD Curves of SC3HO-D (Percent of Tota1 Pore Volume Intruded)

A-8-15 PSD Curves of SC3HD (Percent of Total Pore Volume Existing) . . . . . . . . . . . . . .

A-8-16 PSD Curves of SC3HD (Percent of Total Pore Volume Intruded) .. . . . . . . . . . . .

A-8-17 PSD Curves of OPL (Percent of Total Pore Volume Existing) . . . . . . . . . . . . . . .

A-8-18 PSD Curves of OPL (Percent of Total Pore Volume Intruded) ..... . . . . . . . . . . .

A-8-19 PSD Curves of OPH (Percent of Total Pore Volume Existing) . . . . . . . . . . . . .

A-8-20 PSD Curves of OPH (Percent of Total Pore Volume Intruded) 


\section{LIST OF ABBREVIATIONS}

ASTM American Society for Testing and Materials

PSD Pore Size Distribution

SEM Scanning Electron Microscopy

AASHTO American Association of State Highway and Transportation officials 
SUMMARY

The solls used in this study were a sand and two sand-clay mixtures. Kneading compaction was used to prepare samples of sandclay mixtures while pluvial compaction was used to prepare compacted samples of sand. The testing progran consisted of the permeabilfty test and the pore size distribution (PSD) test. A technique for the preparation of PSD specimens of cohesionless sands has been developed. The mercury intrusion technique was used to conduct PSD test. Falling head tests under back pressure were used to measure permeability. Mathenatical interpretations of PSD were given in detail. For the solls studied, both the pore size distributions and pore size density functions were presented. Pore size density function of sand studled showed a single modal characteristic on a log diameter scale while that of sand-clay mixtures showed bimodal characteristics on a log diameter scale. The influence of varying water content and compactive effort on the fabric of the sandy soils studied was characterized by their pore size distributions and pore size density functions.

A permeability model which related the pore size distribution to the permeability of soils has been proposed. The prediction of permeablitity by the proposed model was excellent $\left(e \cdot g \cdot R_{a}^{2}=0.98\right)$ for 
both the author's and Garcia-Bengochea's (1978) data. In other words, the proposed permeability model can provide an excellent predictive tool. Thus a functional relationship between the PSD and the permeability of compacted soils is clearly demonstrated. 
CHAPTER I INTRODUCTION

\subsection{FABRIC AND ITS ROLE IN GEOTECHNICAL ENGINEERING}

The characterization of soil fabric is a very important aspect of geotechnical engineering. It can lead to better understanding and predi:tion of engineering properties of soils. The term "soil fabric" has widely been used without a common definition, e.g., Terzaghi and Peck (1948), Mitchell (1956), Scott (1963), and Oda (1972). However, the following definition given by Brewer (1964) is preferred:

"Soil fabric is the physical constitution of a soil material as expressed by the special arrangement of the solid particles and associated voids."

Studies at Purdue University, e.g., Diamond (1970, 1971), Bhasin (1975), Reed (1977) and Garcia-Bengochea (1978) among others, have shown that the fabric of compacted clayey soils can be identified by their pore size distribution (PSD) and that some engineering properties of clayey soils can be quantitatively correlated with their PSD. Moreover, the engineering properties of sandy soils are also believed to be affected by their fabric characteristics, e.g., Oda (1972, 1976), Mahmood (1973), and Mitche11, et al. (1976). Figure 1-1 illustrates the concept of sand fabric. Two different arrangements of particles could have identical void ratios and grain size distributions, but their compressibility characteristics would be obviouly different (Leonards, as cited by Holtz and Kovacs, 1981). Similarly, 

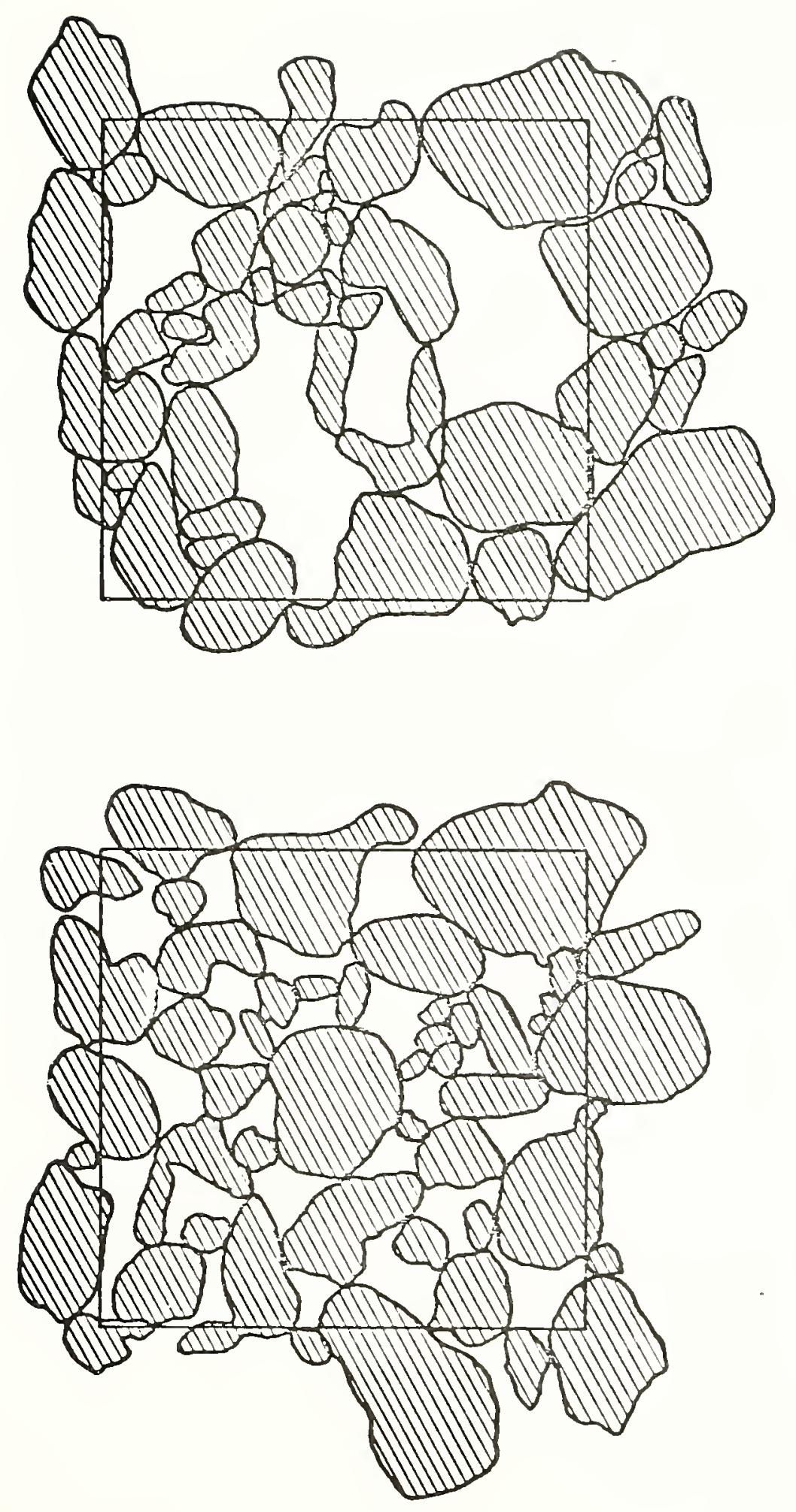

은

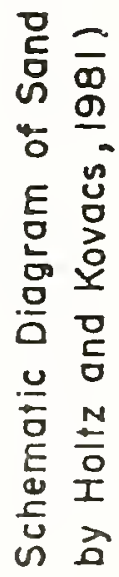

1

选 
the permeability, liquefaction properties, and the static strength of sands are strongly affected by sand fabric.

\subsection{OBJECTIVE OF THIS STUDY}

This study is part of a research project entitled "The Effects of Pore Size Distribution on Permeability and Frost Susceptibllity of Selected Subgrade Materials". The specific objectives are:

1) to determine the fabric characteristics of compacted sand and sand-clay samples by their PSD as measured by the mercury intrusion technique; and

2) to develop a theoretical permeability model which correlates the permeability with the PSD of the selected soils. 
CHAPTER II THEORETICAL BACKGROUND

\subsection{PORE SIZE DISTRIBUTION}

2.1.1 Theoretical Background of Pore Size Distribution and Pore Size Density Function

The pore system has an extremely complex geometry. Therefore, to characterize a soil fabric by means of pore size and its distribution is a rather abstract concept. In fact, there is no clear definition of pore size and therefore its distribution. In the mercury intrusion technique (see Section 2.1.2) a cylindrical pore shape model was assumed, i.e., the circular diameter was considered to be its corresponding pore size.

Based on Washburn's (1921) equation:

$$
P=-\frac{4 T \cos \theta}{d}
$$

where $\mathrm{P}=$ absolute pressure being applied

$$
\begin{aligned}
T= & \text { surface tension of mercury } \\
\Theta= & \text { contact angle between mercury and the pore wall } \\
\mathrm{d}= & \text { the size of pores which can be intruded at the } \\
& \text { pressure } \mathrm{P} \text {; called the apparent pore diameter. }
\end{aligned}
$$

With the assumption of constancy of the surface tension and contact angle, Equation (2-1) implies that as pressure increases, mercury will be intruded into progressively smaller pores. For each pressure increment, mercury is forced into the accessible soll pores of a diameter 
larger than or equal to that which is calculated by Equation (2-1) at the corresponding pressure. Meanwhile the cumulative volume of pore space at any corresponding pressure is also recorded. Then a PSD can be generated (see Equation 2-6d and its interpretation).

Since there is no clear physlcal meaning of pore size, Scheidegger (1957) proposed a probabilistic approach to define a pore system. With slight modification to Scheidegger's ingenious idea and the pioneering work of Drake and Ritter (1945) and Garcia-Bengochea (1978), the PSD and pore size density function can be derived as follow :

Assume $X$ is a random variable denoting a pore diameter at any point within the pore space. It is the diameter of the largest sphere which contains this point and remains wholly within the pore space. Thus, a "pore diameter" can be assigned to each point in the pore space, and a PSD can be defined by determining what fraction of pore space has a pore diameter between $\mathrm{x}$ and $\mathrm{x}+\mathrm{dx}$. (Scheidegger,1957)

Pore size distribution can be expressed as

$$
F_{x}(x)=\int_{x}^{\infty} f_{x}(x) d x
$$

where $F_{x}=$ PSD of the soil being considered

$$
\mathrm{f}_{\mathbf{x}}=\text { the probability density function of pore size }
$$

and

$$
\int_{0}^{\infty} f_{x}(x) d x=1
$$

The probability of pores with diameters ranging from $x$ to $x+d x$ is $f(x) d x$. Now denoting the volume of pores having diameters between $x$ and $x+d x$ as $d V$, we have

$$
d V=s(x) \cdot f_{x}(x) d x
$$


where $s(x)=a$ shape factor and is a function of $x$ (i.e., it depends on the pore shape model chosen).

Recalling Equation (2-1) and noting that the diameter is $x$, we have

$$
P x=\text { constant }
$$

or

$$
d x=-\frac{x}{P} d P
$$

Substituting into Equation (2-3), we have

$$
d V=-s(x) \cdot f_{x}(x) \cdot d P / P \cdot x
$$

or

$$
f_{x}(x)=\frac{P}{x \cdot s(x)} \cdot \frac{d\left(V_{t}-V\right)}{d P}
$$

where $V_{t}=$ total void volume that can be intruded by mercury

$V_{t}-V=a$ measurement of pore volume with diameter equal to or greater than $\mathrm{x}$

$\mathrm{P}=$ corresponding pressure needed to intrude mercury into pores with diameter $\mathrm{x}$.

The terms $x, f_{x}(x), s(x)$ are the same as defined previously in Equations (2-2) and (2-3). Note that the total void volume that can be intruded by mercury is not necessarily the same as that existing in the specimen. Therefore the pore size density functions based on different definitions of $V_{t}$ are different from each other (see Section 4.3.2). Also noce that $s(x)$ is the problem in determining $f_{x}(x)$ of Equation (2-5b). One way to treat it is to introduce the volume pore size density functions $\mathrm{f}_{\mathrm{vx}}(\mathrm{x})$ as follows: 


$$
f_{v x}(x)=s(x) \cdot f_{x}(x)
$$

Substituting Equation (2-5b) into Equation (2-6a), we have

$$
f_{v x}(x)=\frac{P}{x} \cdot \frac{d\left(v_{t}-v\right)}{d P}
$$

Substituting Equation (2-4b) into Equation (2-6b), we have

$$
f_{v x}(x)=-\frac{d\left(v_{t}-v\right)}{d x}
$$

Integrating Equation (2-6c) gives

$$
F_{v x}(x)=v_{t}-v
$$

where $\mathrm{F}_{\mathrm{vx}}(\mathrm{x})$ = the cumulative intruded volume which is directly recorded in the mercury intrusion technique.

So far, the above derivations and definitions provide the theoretical development of pore size distribution and its density function. For any pore diameter which can be calculated from Equation (2-1) with each corresponding pressure being recorded, $\mathrm{F}_{\mathrm{vx}}(\mathrm{x})$ can be obtained from the volume intrusion record. Hence a PSD [i.e., $F_{v x}(x)$ vs. $x]$ can be generated. The pore size density function, $\mathrm{f}_{\mathrm{vx}}(\mathrm{x})$, can then be determined from Equation (2-6c).

The pore size distribution defined above is in terms of pore volume. In many cases, it is convenient to express the PSD in terms of pore volume per gram of specimen. It is also a standard presentation of the PSD test as specified by an ASTM standard being developed by Subcomittee D18.14. Moreover, some researchers prefer to express the PSD in dimensionless terms (e.g., a percentage) for the purpose of convenient mathematical treatment. The following expressions provide a theoretical 
basis for this consideration.

$$
F_{p x}(x)=\frac{P}{x \cdot V_{t}} \cdot \frac{d\left(V_{t}-V\right)}{d P}
$$

where $\mathrm{f}_{\mathrm{px}}(\mathrm{x})$ = density function of pore diameter with percentage as its ordinate

and

$$
F_{p x}(x)=\int_{x}^{\infty} f_{p x}(x) d x
$$

where $\mathrm{F}_{\mathrm{px}}(\mathrm{x})$ = cumulative distribution of pore size (for

$$
\begin{aligned}
& \text { diameter } \geq x \text { ) with percentage as its ordinate; } \\
& \text { this is simply called percent greater by volume. }
\end{aligned}
$$

Furthermore, Equation (2-6b) can also be modified to the following expression:

$$
f_{n x}(x)=\frac{P}{x \cdot V_{s}} \cdot \frac{d\left(V_{t}-V\right)}{d P}
$$

where $V_{S}=$ volume of soil specimen

$$
\begin{aligned}
\mathrm{f}_{\mathrm{nx}}(\mathrm{x})= & \text { density function of pore diameter with a relative frequency } \\
& \text { scale such that }
\end{aligned}
$$

$$
\int_{0}^{\infty} f_{n x}(x) d x=n
$$

where $\mathrm{n}=$ porosity of specimen.

The multiplication of $\mathrm{f}_{\mathrm{nx}}(\mathrm{x})$ by a constant $\mathrm{c}$ (see Section 4.3.2) is called "porosity frequency" by Garcia-Bengochea (1978) and White (1980).

A detailed discussion of the presentation of the PSD test results on the soils studied is given in Section 4.3.2. 
2.1.2 The Measurement of Pore Size Distribution and Its Limitation The measurement of the PSD of a soll is a technique which allows the englneer to indirectly examine the soil fabric. Methods available for the determination of PSD are as follows:

a) Indirect determination from representative grain size distributions by applying probabilistic theory (e.g. Muruta and Sato, 1969);

b) Indirect determination by scanning electron microscopy (SEM) techniques with probabilistic theory;

c) Capillary suction technique;

d) Mercury intrusion technique.

Detalled discussion of all these techniques except (a) are given by Bhasin (1975) and Lowell (1979). Bhasin (1975) had also pointed out that the mercury intrusion technique is the most suitable method for the measurement of the PSD of soils.

The concept of forcing mercury into material pores to determine their sizes was first proposed by Washburn (1921). This led to the development of mercury intrusion technology which is based on the principle that the surface tension of a non-wetting liquid will oppose the entry to the liquid into a small pore of a material. Washburn stated that this opposition could be overcome by external pressure, and that the external pressure required was inversely proportional to the pore diameter, as indicated by Equation (2-1).

Perhaps the primary limitation of the mercury intrusion technique is that it does not always measure the diameter of the actual pore 
but rather the diameter of the channel leading into the pore, which obviously could be smaller. As a result of "1nk-bottle-pore effect", discussed by Ritter and Drake (1945), Orr (1970), and Ahmed (1971) and the other Purdue researchers, the measured pore size is called the limiting pore diameter or called apparent pore diameter (e.g., by a proposed ASTM standard D18.14). An attempt has been made by Cebeci, et a1. (1978) to evaluate the arrangement of ink-bottle pores. However, there is still no way to obtain the "true" pore size distribution. This problem is of minor impor: ance in the case of flow through porous materials, since the pore channels the water has to pass through are probably equivalent to those for mercury.

The second problem with mercury intrusion technology is that cylindrical pores are assumed. Bhasin (1975) pointed out that this assumption may be fairly reasonable since the difference in calculated PSD's due to different assumptions of pore shapes are relatively smal1 (i.e.,within an order of magnitude) when compared with the range of pore sizes that are possible (i.e., up to five orders of magnitude).

Another limitation is that mercury intrusion porosimetry, in common with many other methods, is only capable of intruding pores that are open to the outside of a soil specimen. The volume of any pores that are completely enclosed by surrounding solids cannot be determined. Moreover, the method will only determine the volume of intrudable pores that have an apparent diameter corresponding to a pressure within the pressuring range of the testing instrument.

Further discussion of the assumptions and sources of error of the mercury intrusion technique, including the concern of whether or not 
the soil structure breaks down during intrusion, the assumptions of constant contact angle and surface tension values, non-reaction of the mercury with the soil, and incompressibility of the soil fabric, among others, is found in Ritter and Drake (1945), Rootare (1968), Winslow (1969), Diamond (1970), Bhasin (1975), Garcia-Bengochea, et a1. (1980), Kenney (1980), Reed, et a1. (1980), and a proposed ASTM standard, subcommittee D18.14.

2.1.3 The Applications of Pore Size Distribution

To predict the permeability of porous materials is one of the early applications of the PSD. As is well known, seepage occurs only through the pores, and what is directly related with the permeability are the characteristics of pores themselves. Moreover, permeability is extremely sensitive to subtle changes in soil structure and these changes are not accurately reflected by the bulk volumetric pore parameters (e.g., the porosity or void ratio of the sample). This led to the attempts to use PSD to predict the permeability of porous material. Among those are Childs and Collis-George (1950), Marshall (1958), Millington and Quirk (1959), Kunze, et a1. (1968), Murota and Sato (1969), Klock, et al. (1969), Green and Corey (1971), Jackson (1972), and Garcia-Bengochea, et al. (1979). However, only the last reference clearly demonstrated the usefulness of the PSD measurements on interpreting changes in soil fabric and predicting how these changes might affect the permeability of soils.

Another application of PSD is to predict the frost heave rate (or amount) in soils. References include Csathy and Townsend (1962), Zoller (1973), Gaskin and Raymond (1973), and Reed, et al.. (1979). 
Among other applications, Lohnes, Tuncer and Demirel (1976) related PSD to weathering and mineralogy of loess soils. Ahmed, et al. (1974) related PSD to the strength of clayey soils.

Finally, based on PSD studies at Purdue University during the past decade, an understanding of the mechanism of compressibility and that of liquefaction of sandy soils may be possible.

\subsection{PERMEABILITY MODEL}

2.1.1 Relation of Permeability to Pore Geometry

Since permeability is a characteristic physical property of a porous medium, it would seem only reasonable to assume that it is related in some functional way to certain measureable properties of the soil pore geometry, e.g., porosity, pore size distribution, internal surface area, etc. However, numerous attempts to discover a functional relation of universal applicability have so far met with disappointing results (Hillel, 1980).

Perhaps the simplest approach is to seek a correlation between permeabilfty and porosity (or void ratio). However, Scheidegger (1957) and Hillel (1980), among others, concluded that there is no simple correlation between permeability and porosity because of the strong dependence of flow rate on the width, continuity, shape, and tortuosity of the conducting channels. Thus, a medium composed of numerous small pores with a high total porosity is likely to exhibit a lower saturated permeability than a medium composed of fewer large pores with a lesser porosity.

Prediction of permeability from grain size distribution has also been used for a long time (Hazen, 1911). Such predictive equations 
have had only limited success for coarser solls and have proved to be unsatisfactory for fine-grained solls. Efforts to refine this approach by introducing empirical grain shape and packing parameters have not won general acceptance (Hillel, 1980).

Numerous theoretical models have been introduced to represent porous media by a set of relationships that are amenable to mathematical treatment. Basically, there are two main groups of those models. The first is based on a generalization of Kozeny's approach (Carman, 15j6) for saturated and unsaturated porous media. This theory is based on the concept of a hydraulic radius, i.e., a characteristic length parameter presumed to be linked with the hypothetical channels to which the porous medium is thought to be equivalent. The derivation and critique of this theory can be found in Scheidegger (1957).

The second group employed the capillary model using the PSD to calculate permeability. Scheidegger (1957) gave a comprehensive review of these models, including the straight capillaric, parallel, serial, and branching models. He pointed out that, in general, natural porous media are extremely disordered, so that it seems a rather poor procedure to represent them by something which is intrinsically ordered. He therefore suggested that the preferred model of a porous medium should be based on statistical concepts.

A statistical flow model, as shown in Flgure 2-1, was first presented by Childs and Collis-George (1950) and later modified by Marshall (1958) and Millington and Quirk (1959). Conceptually, their approach rests on the assumptions that ( 1 ) a soil contains distinct pores of various sizes which are randomly distributed in space, and (2) when adjacent sections of the soll are brought into contact, the 


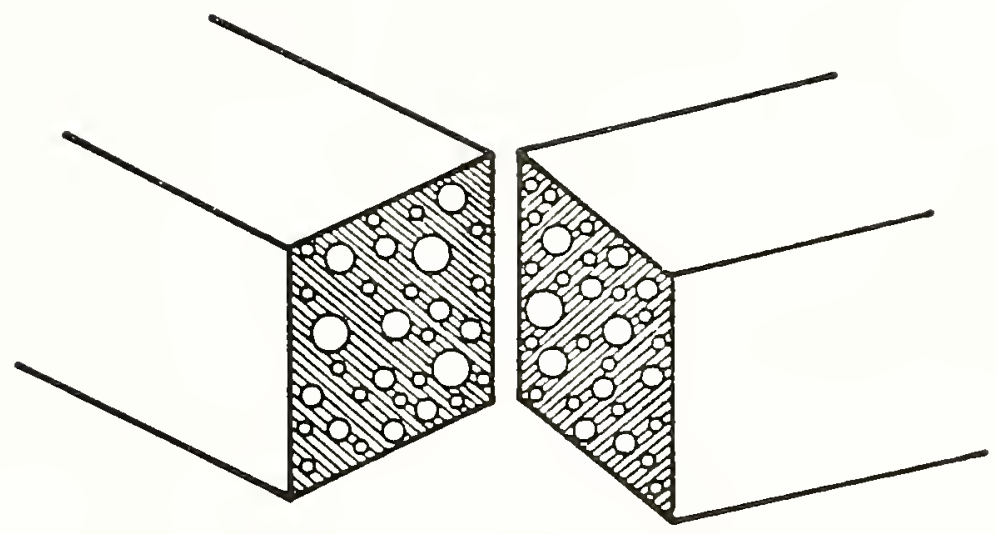

Figure 2-1 Schematic Representation of a Sectioned Soil

(After Hillel, 1980) 
overall quantity of flow across the section depends statistically on the number of pairs of interconnected pores and geometrically on their configurations. The quantity of flow of each pair of interconnected pores 1 determined by the narrower of the two pores.

The original equation by Childs and Collis-George (1950) was

$$
k=c \frac{\rho g}{\mu} \sum_{s=0}^{s=R} \sum_{\delta=0}^{\delta=R} \delta^{2} f(s) \text { ds } f(\delta) d \delta
$$

where

$$
\begin{aligned}
\mathrm{k}= & \text { permeability } \\
\mathrm{c}= & \text { a matching constant } \\
\rho= & \text { density of water } \\
\mathrm{g}= & \text { gravitational acceleration } \\
\mu= & \text { coefficient of absolute viscosity of water } \\
\mathrm{f}(\mathrm{s}) \mathrm{d} s= & \text { the partial pore space occupied by pores with radii } \\
\mathrm{f}(\delta) \mathrm{d} \delta= & \text { the partial pore space of pores with radii of } \\
& \delta \rightarrow \delta+d \delta \\
\mathrm{R}= & \text { maximum pore radius }
\end{aligned}
$$

Note that although theoretically the calculation based on Polseuille's law does not require a matching factor, in actual practice a matching factor is needed to adjust the computed and measured permeability at full saturation. The method of calculation was subsequently improved by Marshall (1958), Millington and Quirk (1959), Kunze et al. (1968), Green and Corey (1971), and Jackson (1972). Basically these developments are different only by some minor arbitrary factors and the ways of calculation. Moreover, Garcia-Bengochea (1978) presented a somewhat different equation based on regression analysis of his data as follows: 


$$
\mathrm{k}=\mathrm{c}_{\mathrm{s}} \cdot \mathrm{PSP}^{\mathrm{b}}
$$

where $\quad b=$ parameter of regression $c_{s}=$ a constant, called shape factor

and

$$
\operatorname{PSP}=\sum_{\substack{i \\ i}}^{n} \tilde{d}^{2} f\left(d_{i}\right) \quad f\left(d_{j}\right)
$$

$$
\begin{aligned}
\widetilde{d}= & \text { smaller of } d_{i} \text { and } d_{j} \\
f\left(d_{1}\right), f\left(d_{j}\right)= & \text { volumetric frequency of occurrence of pores with } \\
& \text { diameter of } d \rightarrow d+\Delta d \text {; conceptually it equals } \\
& f(s) d s \text { or } f(\delta) d \delta \text { in Equation }(2-9) .
\end{aligned}
$$

As noted by Garcia-Bengochea, et a1. (1979), as b $\rightarrow 1$, Equation (2-10) becomes the Child and Collis-George's (1950) model. Since experimentally $b$ ranges from 1.67 to 4.95 , Equation (2-10) is indeed an empirical equation.

\subsubsection{Proposed Permeability Model}

From the previous discussion, it is clear that Child and CollisGeorge's (1950) model and its modifications are more generally applicable than those based on earlier models. However, an arbitrary matching factor or regression parameter, as appears in Equation (2-9) and Equation (2-10) respectively, is necessary to reduce or to eliminate the deviations between predicted and measured permeability. One of the objectives of the present study is to propose a modified model which eliminates the deviation without the necessity of an arbitrary factor or parameter.

Considering a homogeneous soil having interconnected pores which are randomly distributed in space, the probability of pores of diameter 
$x \rightarrow x+d x$ is $f(x) d x$, where $f(x)$ is the pore size density function of the soil. Note that no assumption is needed for $f(x)$. However, in the present study, $f(x)$ is defined by Equation $(2-7 a)$. The term $f(x) d x$, represents the ratio between the pore volume occupied by pores of diameter $\mathrm{x} \rightarrow \mathrm{x}+\mathrm{dx}$ and the total pore volume. Note that if a cross section through soil mass is considered, the above volumetric ratio is equal to the areal ratio between the pore area of diameter $x \rightarrow x+d x$ and the total pore area.

Now, consider a soil column with a thickness $\Delta y$ and two cross sections with an identical pore size density function (see Figure 2-2). The probability of pores of diameter $x_{i} \rightarrow x_{i}+d x_{i}$ on cross section $i$ being connected to pores of diameter $x_{j} \rightarrow x_{j}+d x_{j}$ on cross section $j$, $P\left(x_{i}, x_{j}\right)$, has the following extreme cases:

(1) For $\Delta y \gg x$, the connections between pores on the two cross sections can be reasonably assumed to be completely random. Hence

$$
P\left(x_{i}, x_{j}\right)=f\left(x_{i}\right) f\left(x_{j}\right) d x_{i} d x_{j}
$$

(2) For $\Delta y \rightarrow 0$, the connections between pores considered are completely correlated.

Hence

$$
P\left(x_{i}, x_{j}\right)=f\left(x_{i}\right) d x_{i} \text { or } f\left(x_{j}\right) d x_{j}
$$

For case 1, following the similar derivation by Childs and Collis-George (1950) or Garcia-Bengochea (1978), we have

$$
k=\frac{y n^{2}}{32 \mu} \int_{0}^{\infty} \int_{0}^{\infty} \bar{x}^{2} f\left(x_{i}\right) f\left(x_{j}\right) d x_{i} d x_{j}
$$




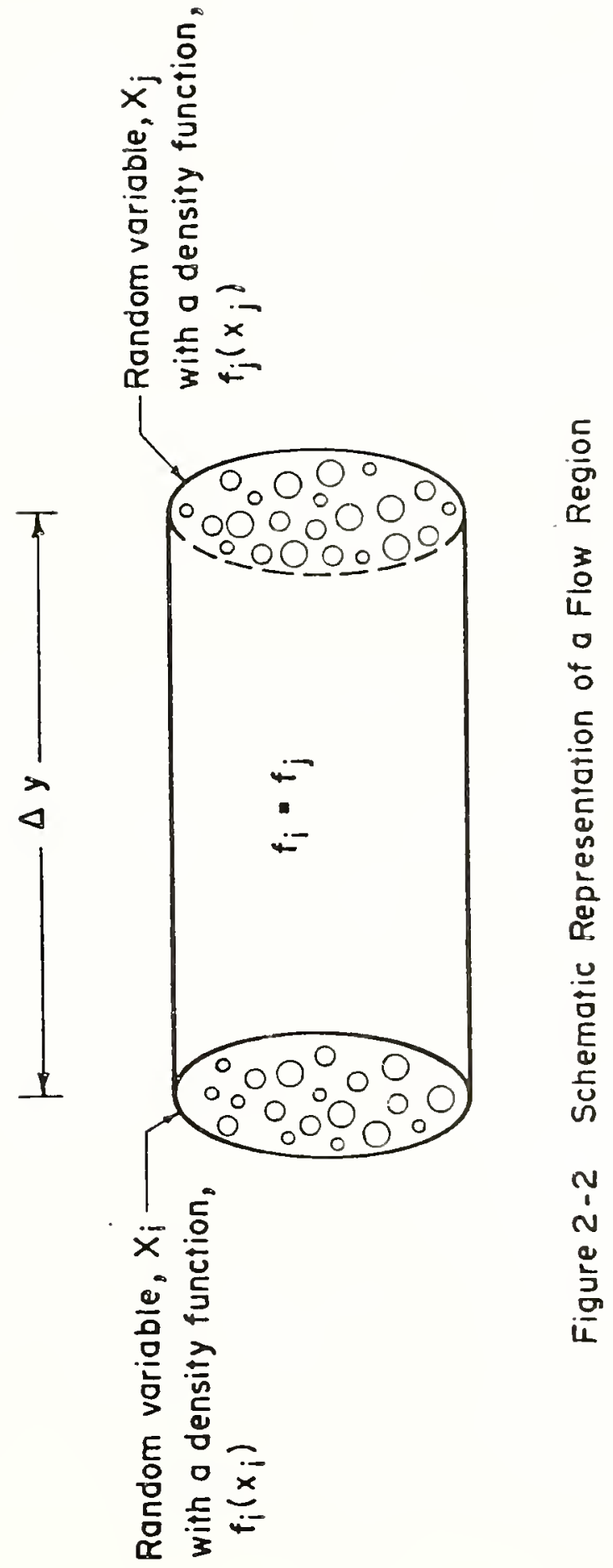


where $\gamma=$ unit weight of water

$$
\begin{aligned}
& \mu=\text { coefficient of absolute viscosity of water } \\
& \mathbf{n}=\text { porosity of soil } \\
& \tilde{\mathbf{x}}=\text { equivalent diameter of capillary element. }
\end{aligned}
$$

Equation (2-13) is essentially equal to Equation (2-9) except that no matching factor is provided. This equation is also called the Marshall model by Garcia-Bengochea (1978).

For case 2 , in a similar manner, we have

$$
k=\frac{y n^{2}}{32 \mu} \int_{0}^{\infty} x^{2} f(x) d x
$$

Equation (2-14) is called the capillary model by Garcia-Bengochea (1978) .

Since we are concerned with the effect of pore geometry changes on the permeability, it is more relevant to consider $\Delta y$ to be of the same order of magnitude as the pore diameter. Therefore the connections between pores on the two cross-sections are partially correlated.

A reasonable assumption to account for the partially correlated connections is that pores of diameter $x_{i} \rightarrow x_{i}+d x_{1}$ on cross-section $i$ have a greater probability of being connected to pores of the same diameter (i.e., $x_{i} \rightarrow x_{i}+d x_{1}$ ) on cross-section $j$, than that of being connected to the other different-sized pores on cross-section $j$. Based on this idea, a connection rule is illustrated in Figure 2-3.

The probability of pores of diameter $x_{i} \rightarrow x_{i}+d x_{1}$ on crosssection $i$ being connected to pores of diameter $x_{j} \rightarrow x_{j}+d_{j}$ on crosssection $j$ is

$$
P\left(x_{1}, x_{j}\right)=g\left(y, x_{1}, x_{j}\right) \cdot f\left(x_{i}\right) \cdot f\left(x_{j}\right) d x_{1} d x_{j}
$$




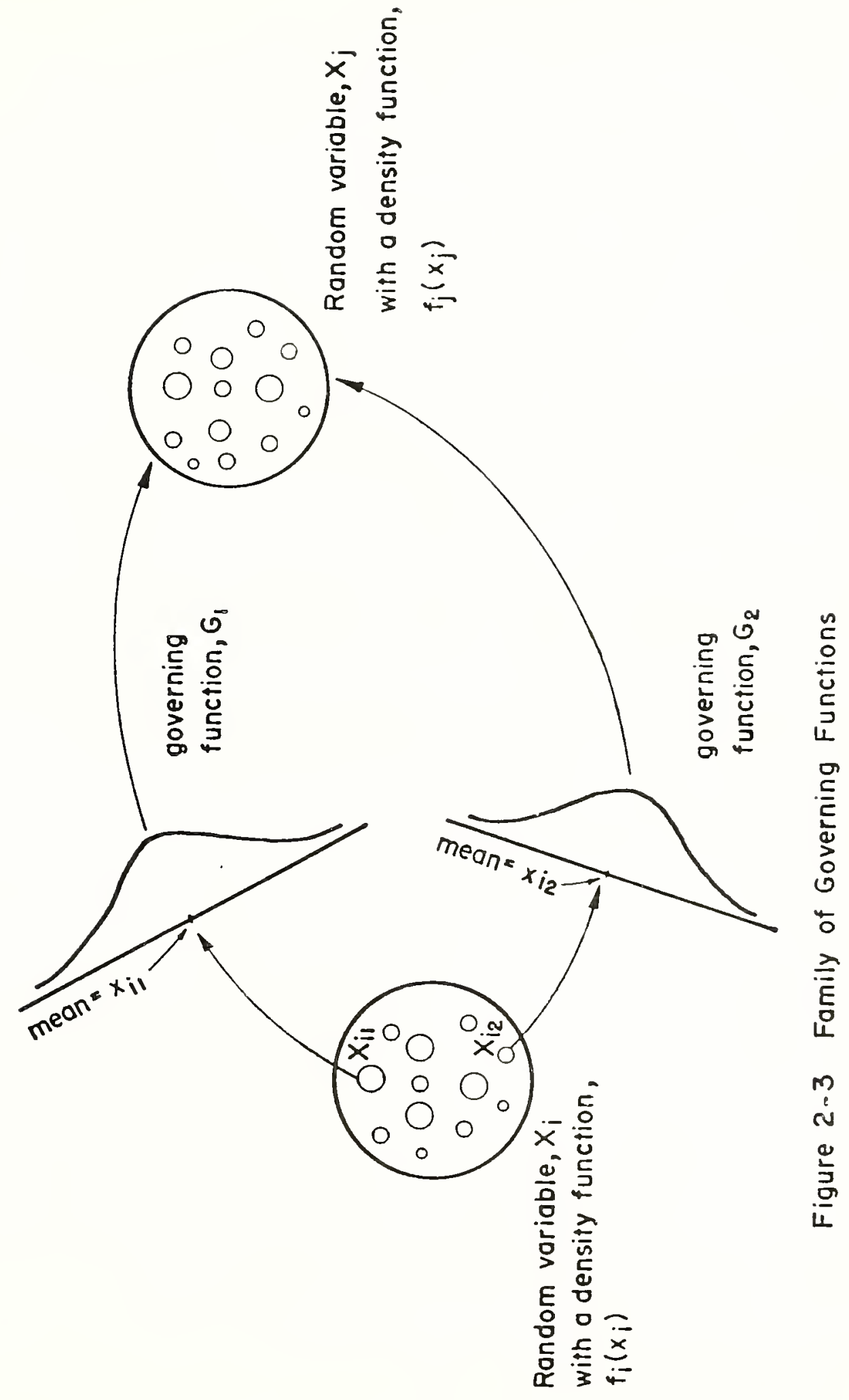


and

$$
k=\frac{y n^{2}}{32 \mu} \int_{0}^{\infty} \int_{0}^{\infty} \tilde{x}^{2} \cdot g\left(y, x_{i}, x_{j}\right) \cdot f\left(x_{i}\right) \cdot f\left(x_{j}\right) d x_{1} d x_{j}
$$

where $g\left(y, x_{i}, x_{j}\right)=$ connecting function and

$$
y=\text { the third directional variable (length). }
$$

If we also consider a correction factor, $t\left(y, x_{i}, x_{j}\right)<1$, to account for the tortuosity of flow, then the permeability becomes

$$
k=\frac{\gamma n^{2}}{32 \mu} \int_{0}^{\infty} \int_{0}^{\infty} \tilde{x}^{2} \cdot g \cdot t \cdot f\left(x_{i}\right) \cdot f\left(x_{j}\right) d x_{i} d x_{j}
$$

Based on the above development, we now introduce a governing function $G\left(x_{j}\right)$ to account for $g$ and $t$ as follows (see Figure 2-3)

$$
G\left(x_{j}\right)=e^{-\left(\frac{x_{j}-x_{i}}{v}\right)^{2}}
$$

where $V=V\left(x_{j}\right)$

$=$ an undetermined function of pore size to account for the third directional variable and the tortuosity of flow. Note that pore size density functions $f\left(x_{i}\right)$ and $f\left(x_{j}\right)$ are identical. And random variables $X_{i}$ and $X_{j}$ are symetrical in Equation (2-18).

For any pores of diameter $x_{i} \rightarrow x_{i}+d x_{i}$, the governing function is bell-shaped and symmetrical with a mean value of $x_{i} \rightarrow x_{i}+d x_{1}$, as shown in Figure 2-3. Therefore Equation (2-18) is a family function and each member of the family is determined by a pair of parameters, i.e., $\mathrm{X}_{i}$ and $\mathrm{V}$.

Based on the above discussion, Equation (2-17) can be refined as follows:

$$
k=\frac{y n^{2}}{32 \mu} \int_{0}^{\infty} \int_{0}^{\infty} \tilde{x}^{2} \cdot G\left(x_{j}\right) \cdot f\left(x_{1}\right) \cdot f\left(x_{f}\right) d x_{1} d x_{j}
$$


Equation (2-19) is the proposed permeability model which has the following characteristics:

(1) a probabilistic basis;

(2) no arbitrary matching factor required; and

(3) a tortuosity factor being considered. 
CHAPTER III TESTING PROGRAM AND EXPERIMENTAL PROCEDURES

\subsection{TESTING PROGRAM}

The testing program was designed to accomplish the objectives mentioned in section 1.2. The flow chart of the program is shown in Figure 3-1. The soils were selected in order to extend the results of the previous permeability-pore size distribution study at Purdue University by Garcia-Bengochea (1978), i.e.,to extend the prediction model to wider ranges of permeability and to characterize different fabrics of sandy soils. The compaction variables considered in this study are water content, energy level, and compaction method.

The pore size distributions (PSD) of the selected soils were determined by the mercury intrusion method. For clay-sand mixtures, fullowing the permeability test, the PSD specimens were trimmed and dried by a freeze-drying technique and were made ready for the PSD test. For pure sand, the problem of determining its pore size distribution is in sampling, i.e., how to get a PSD specimen without destroying its fabric. In the present study, this problem has been solved by using a resin as a bonding material. A small amount, $0.5 \%$ by weight, of Varcum 1364 phenolic resin made by Reichhold Chemicals, Inc. was mixed with sand before compaction. After compaction the entire sample and mold were placed in the oven at a temperature of $170^{\circ} \mathrm{C}$ for $1 \mathrm{~h}$. The sample was then removed and placed in a desiccator several hours until it cooled. The oven-dried. compacted samples were found to have 


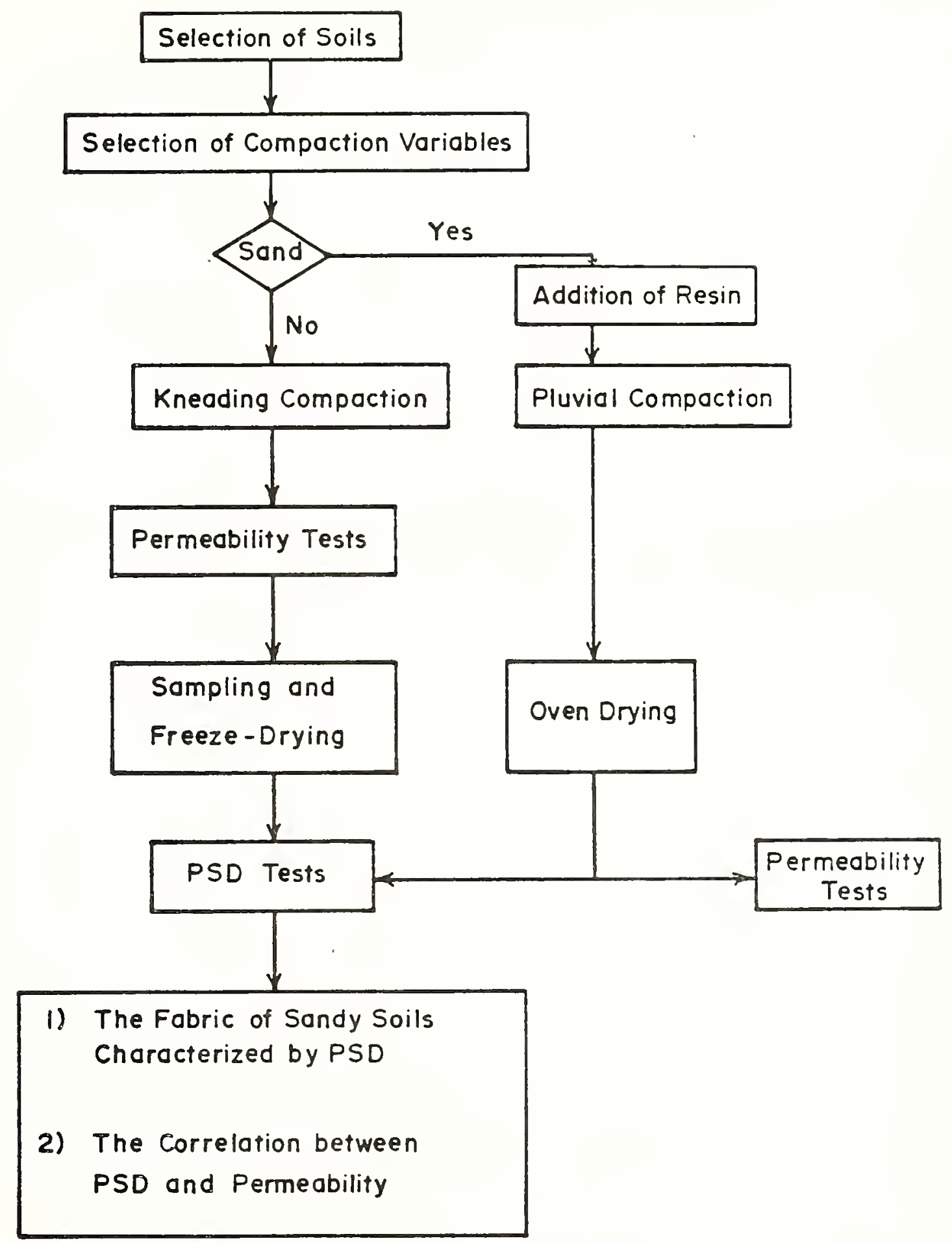

Figure 3-1 Flow Chart of the Testing Program 
about $5 \%$ shrinkage in volume but no change in total weight. Winslow(1980) stated that no chemical reaction between sand and powdered phenolic resin occurs during oven-drying because the resin only acts as a glue. Moreover, the results of the PSD tests on specimens trimmed from these oven-dried samples are reproducible. Therefore this technique is believed to be the best way to obtain PSD specimens of pure sand. During some preliminary work, the minimum amount of resin which provides sufficient bonding and workability was found to be $0.5 \%$ by weight of sand.

The following sections desrribe the soils studied, compaction methods, permeability tests, specimen drying methods, and the PSD tests.

\subsection{SOILS STUDIED}

Sand and sand-clay mixtures used in this study have been arbitrarily called Soils A, B, and C, respectively. The specifications of these three soils are given in Table 3-1. The clay used is Edgar plastic kaolin with the following index properties (Garcia-Bengochea, 1978): specific gravity of solids $=2.65$, liquid Iimit $=59 \%$, plasticity index $=22 \%$, and the clay fraction (\% less than $2 \mu m$ ) $=76 \%$. The sand used is a uniform Ottawa sand. The grain size distribution curves and the classification properties of Soils A, B, and C are shown in Figure 3-2 and Table 3-1 respectively.

\subsection{COMPACTION}

\subsubsection{Pluvial Compaction for Soil A}

For Soil A, $0.5 \%$ powdered phenolic resin was mixed with Ottawa sand before compaction. Pluviation was used to compact the sample in a standard Proctor compaction mold (ASTM D698) with a diameter of 


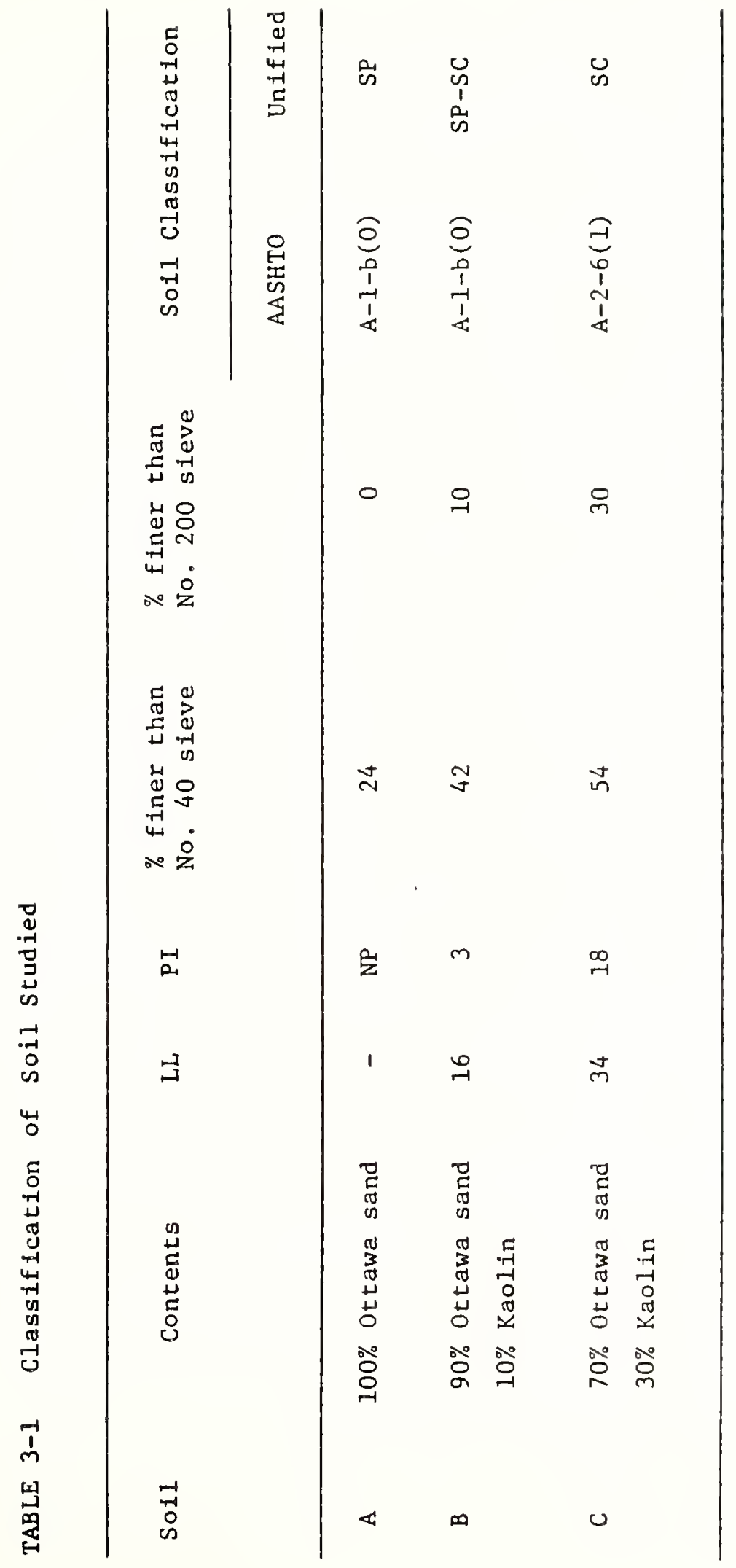




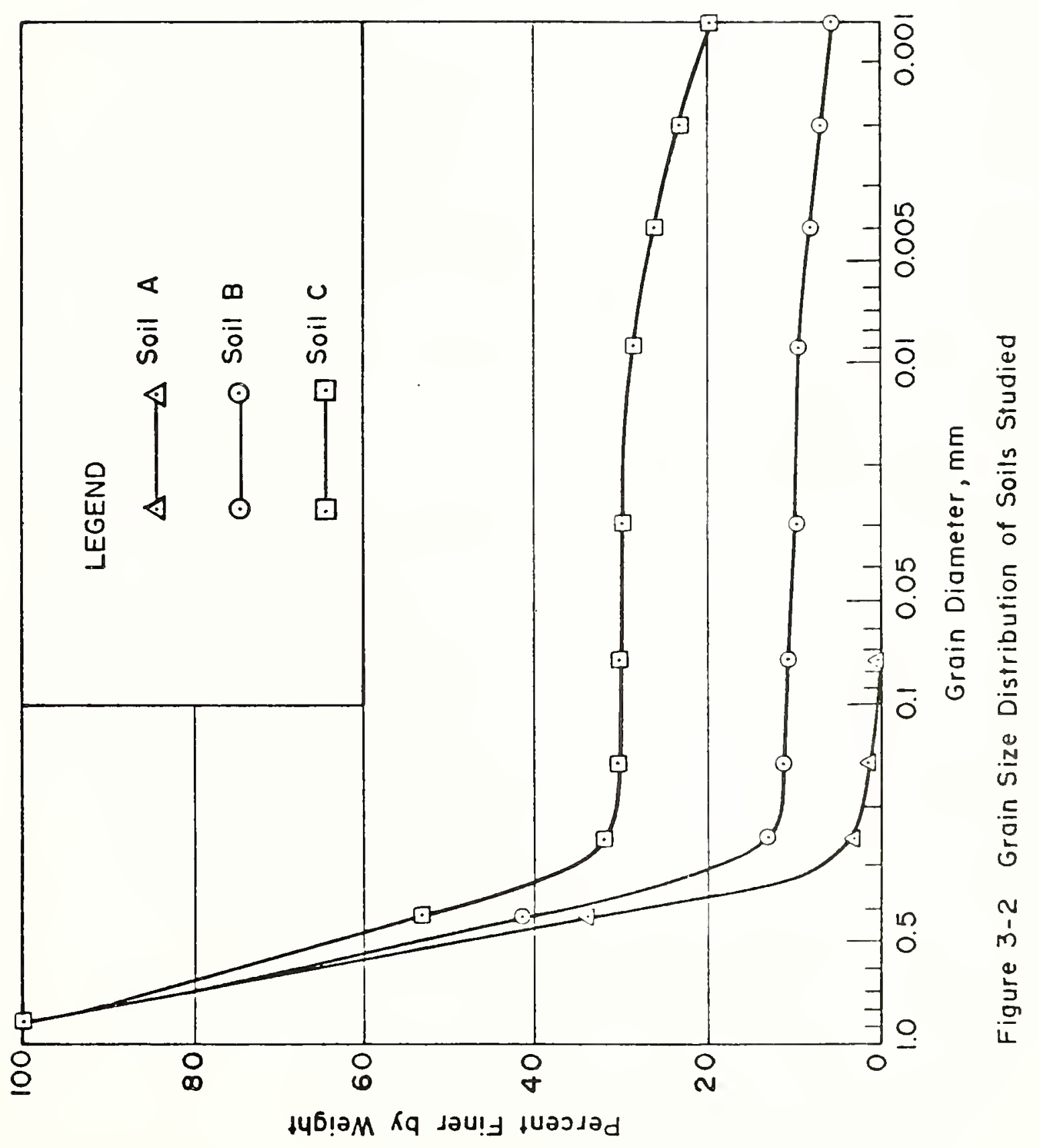


$102 \mathrm{~mm}$ and $116 \mathrm{~mm}$ high. Figure 3-3 illustrates the scheme of the pluviation. The sand-resin mixture was poured from a $1000 \mathrm{ml}$ flask through a nozzle made from rubber stopper containing one hole of either 6.8 or $3.8 \mathrm{~mm}$ diameter. The sand-resin mixture was free to fall to the surface of the sample while the flask was rotated at a rate of approximately $15 \mathrm{rpm}$. The density of the sample obtained was a function of the intensity of particle rain, which, in turn, depended on hole size, and the dropping height. In the present study, the sample was made in five layers with height of drop for each layer being $108 \mathrm{~mm}$. In order to keep the same height of drop, the position of flask was adjusted for each layer. The choice of dropping height and hole size depended on the density of the compacted sample desired. For this study, they were selected by trial and error.

\subsubsection{Kneading Compaction for Soils $B$ and $C$}

An electrically driven semi-automatic kneading compactor shown in Figure 3-4 was used to compact the samples in a standard Proctor compaction mold. The compaction mold was also used as the permeameter cell in order to reduce the possibility of seepage along the walls of the mold. The kneading compaction procedures described by GarciaBengochea (1978) were used in this study with the exception of the definition of the energy level. In the present study, the density level was defined by the standard and modified Proctor compaction as speciFled in ASTM D698 and D1557. The gage pressure set on the keneading compactor at each water content was selected by trial and error in an attempt to obtain the same density as indicated on the Proctor compaction curves. In Garcia-Bengochea's (1978) study, the gage pressure 


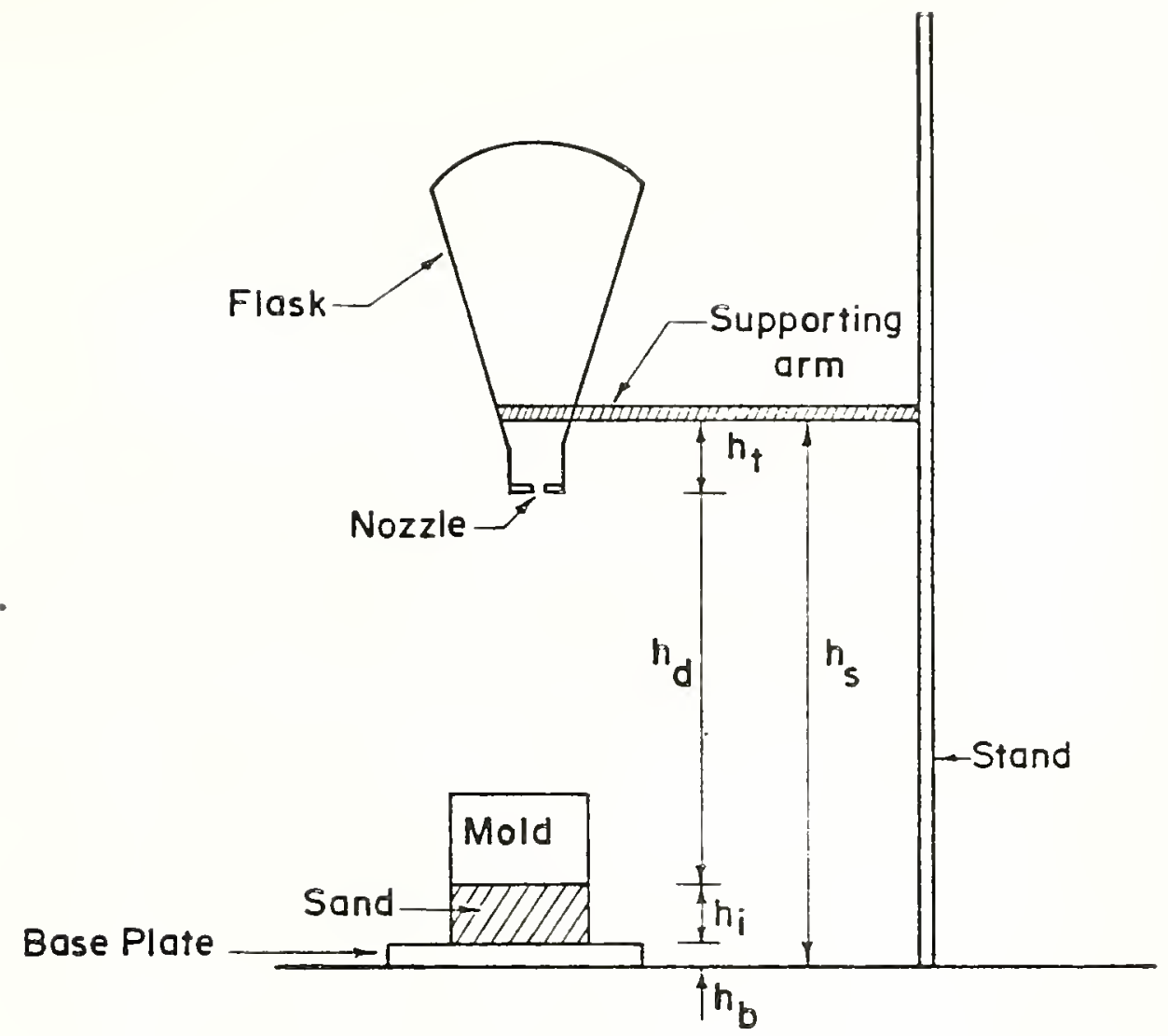

Height of Drop, $h_{d}$

$$
h_{d}=h_{s}-h_{f}-h_{b}-h_{i}
$$

Where

$$
\begin{aligned}
& h_{s}=\text { adjustable height } \\
& h_{i}=\text { height of sand being filled ofter } \\
& \quad(i-1)^{\text {th }} \text { filling; } h_{i=1}=0 \\
& h_{f}, h_{b}=\text { constants, as shown in figure }
\end{aligned}
$$

Figure 3-3 Schematic Diagram of Pluviation System 


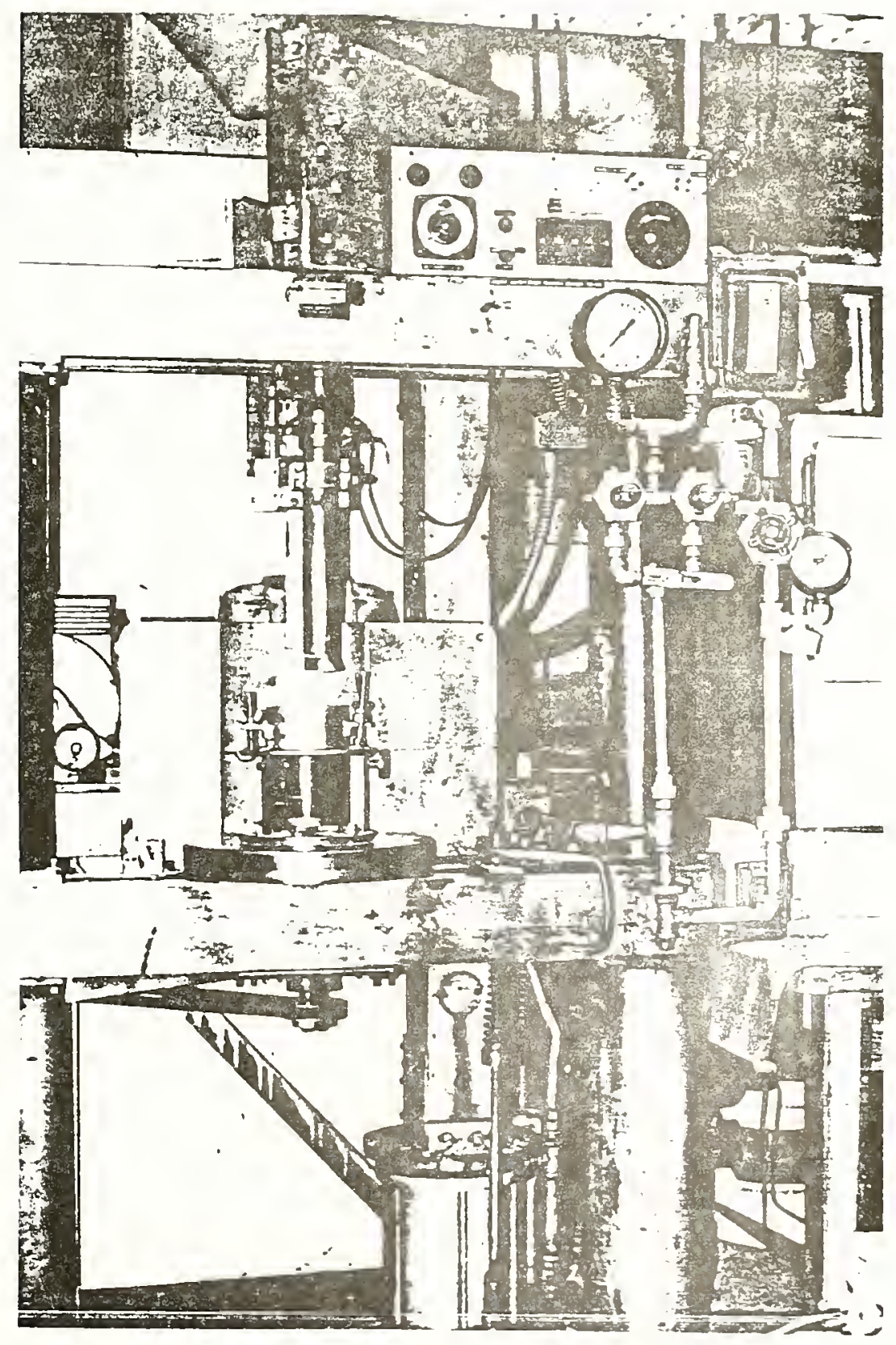

Figure 3-4 Kneading Compactor 
was set to the same value for each water content to define an energy level, as shown in Figure 3-5. The reasons for the present approach are (1) the density-water content curves for different compaction methods may not have the same shape, and (2) the Proctor compaction curve is more common in soils engineering practice for specifying compaction energy. Following the present procedure, calibration curves for converting gage pressure to the actual foot pressure are not necessary. Hence the problem of variation of calibration curves by seasonal changes of temperature and humidity, as noted by Garcia-Bengochea (1978), has been eliminated.

After mixing and a one day curing period, the soil was placed in the Proctor mold and compacted in five layers of approximately equal thickness. Thirty tamps of the compaction foot were applied for each layer. Prior to compaction of each layer except the first one, the previous layer was scarified, and then fresh soil was added. The scarification of the layers prevented separation between the layers. Once the compaction procedure was completed, the collar of the mold was removed and the soil sample was trimmed flush with the top of the Proctor mold.

\subsection{PERMEABILITY TESTS}

Basically, the permeability testing equipment and procedures used in this study followed Garcia-Bengochea (1978).

For soil A, the compacted sample in the Proctor mold was connected to the saturation and permeation device after oven drying. For soils $B$ and $C$, the sample in the mold was connected to the saturation and permeation device directly after completion of compaction process. 


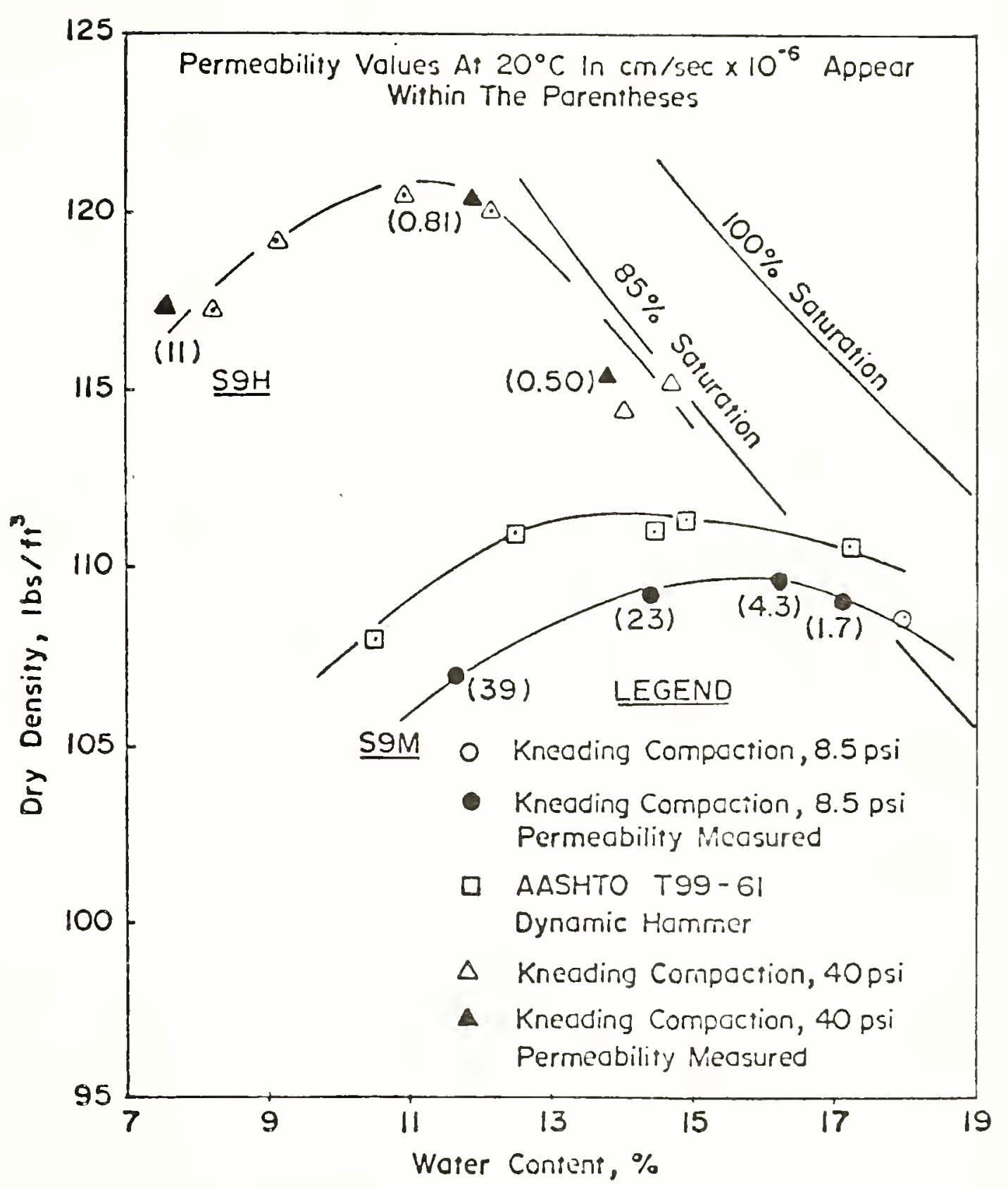

Figure 3-5 Compaction Curves for $90 \%$ Silt and $10 \%$ Koolin

(After Garcio-Bengochea, 1978) 
The saturation and permeation device is a falling head permeameter system with provisions for applying both vacuum and back pressure. The apparatus shown in Figure 3-6 consists of three air pressure regulators, one air pressure gage, two standpipes and air pressurc and vacuum lines. The two permeameters are nearly identical except for the inside diameters of the standpipes. The permeameter used for measuring the more permeable soils has $12.7 \mathrm{~mm}$ inside diameter (I.D.) standpipes, while the other has $6.3 \mathrm{~mm}$ I.D. standpipes. The standpipes can be pressurized simultaneously from one or independently from two regulators. Meter sticks were used to record the water elevation heads. The device is designed for both saturation and permeation. A permeameter cell is shown in Figure 3-6. For soll C, a vacuum of about $34.5 \mathrm{kPa}$ (5 psi) absolute pressure was placed on the top of the sample while the bottom end was under approximately $1 \mathrm{~m}$ of water head. This condition was maintained for about 10 hours which allowed de-aired water to flow upward through the sample and allowed air bubbles to escape. For soils $A$ and $B$, due to their relatively high permeabilities, the above vacuum-saturation process was accomplished within an hour.

After vacuum saturation, back pressure was applied in increments of $34.5 \mathrm{kPa}$ ( $5 \mathrm{psi}$ ) to both ends of the sample. Each increment was maintained for 1.0 minutes. When the back pressure reached $345 \mathrm{kPa}$ (50 psi), it was maintained for about 15 hours.

In the above saturation process, no B-check is possible to assure that the sample is fully saturated. However, as noted by GarciaBengochea (1978), the vacuum saturation consistently resulted in a saturation level of at least $95 \%$ for the clay-silt mixtures. There is no measurement of the degree of saturation after vacuum saturation. Accord- 


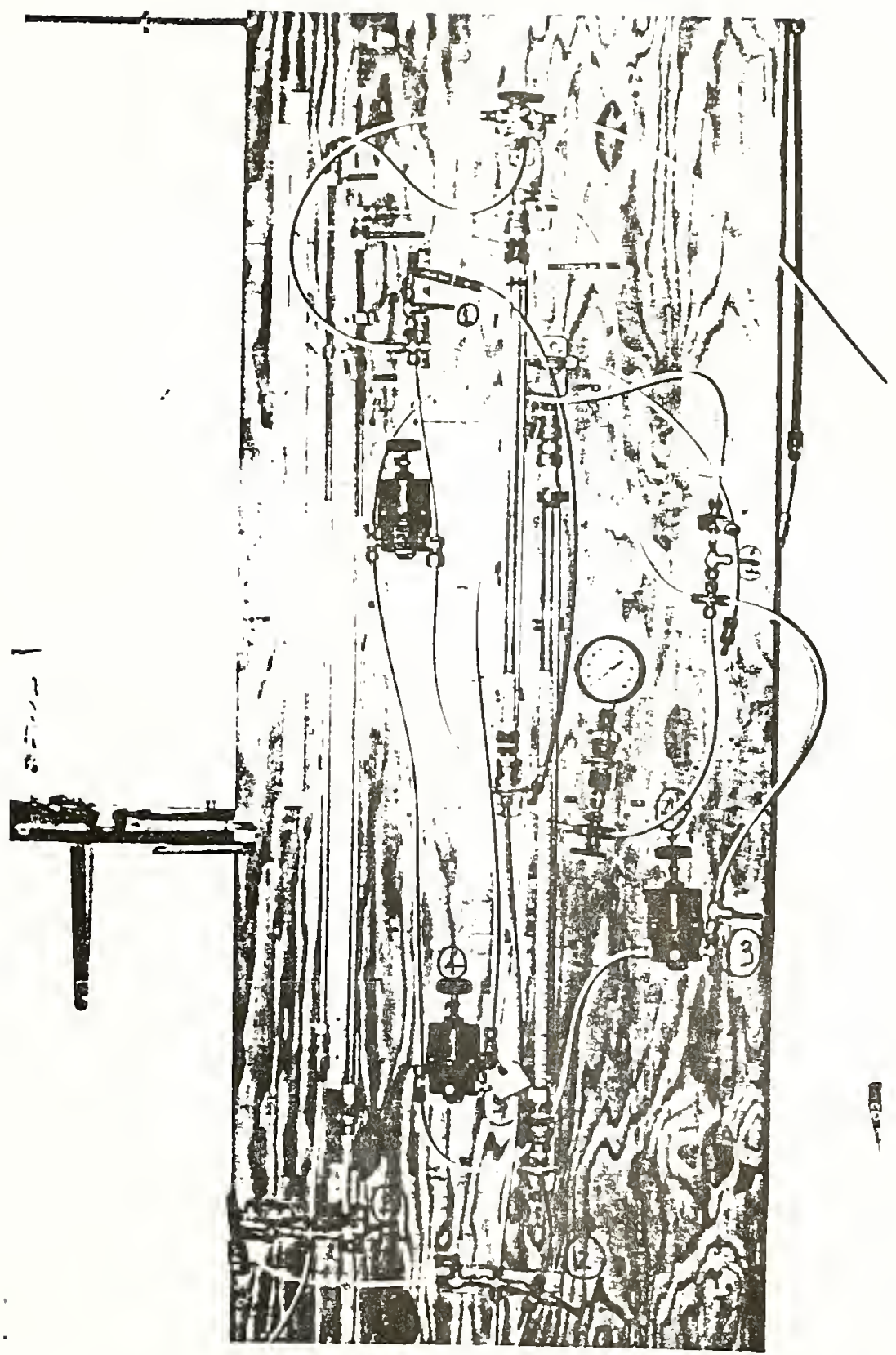

Figure 3-6 Saturation and Permeation Device and Permeameter Cells 


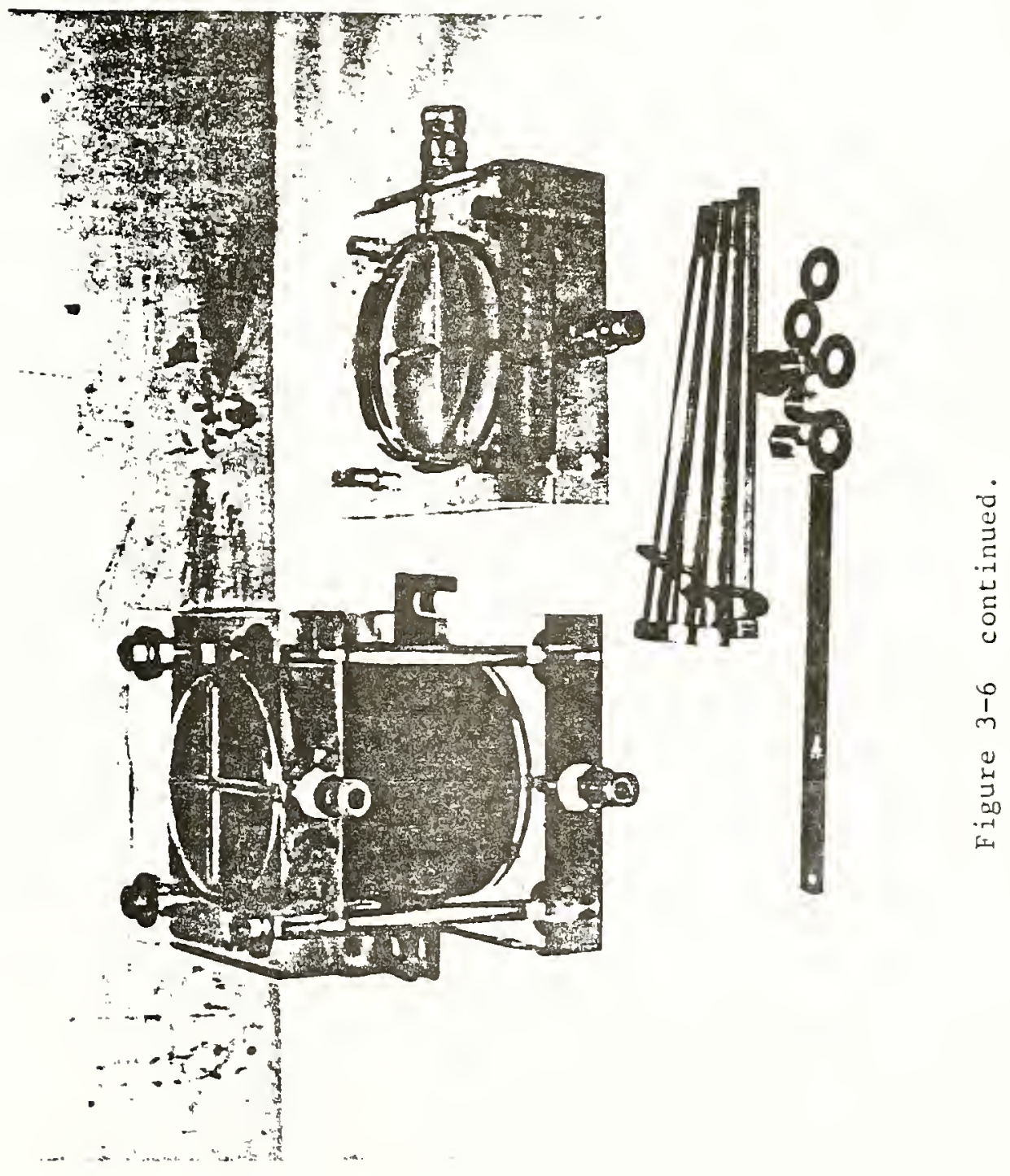


Ing to Black and Lee (1973), the back pressure required to reach a $99 \%$ saturation is about $300 \mathrm{kPa}$ ( $44 \mathrm{psi}$ ) for initial degree of saturation of $95 \%$. The corresponding time required to reach equilibrium is about 4 hours. For the saturation procedure described above, it is believed the degree of saturation is at least $99 \%$, which is appropriate for the practical purposes of permeability measurement. To check this inference, a sample was tested following the above procedures except that a back pressure of $483 \mathrm{kPa}$ (70 psi) was applied during the saturation and permeation phases. No measureable difference was found between the measured permeabilities.

At the completion of the saturation procedure, the falling head permeability tests were conducted. The direction of flow was from top to bottom across the sample. A initial head difference of about $100 \mathrm{~cm}$ or $45 \mathrm{~cm}$ was created between the two standpipes while a $345 \mathrm{kPa}$ (50 psi) back pressure was maintained on both standpipes. Readings of the elevation heads in the water columns, time, and air temperature was recorded.

Following permeation, the sample was dissected to check for unifomity and trimmed for freeze drying and pore size distribution tests. As noted by Garcia-Bengochea (1978), the back pressure on the sample should not be released too rapidly in order to prevent possible sample disturbance. Thus each decrement of $34.5 \mathrm{kPa}$ (5.psi) was maintained for 15 minutes. When atmospheric pressure was reached, the sample was left in the mold for at least 2 hours before dissection and specimen trimming. 


\subsection{SPECIMEN PREPARATION AND DRYING AND PSD TESTS}

For Soll A, specimens for the PSD test were trimmed from ovendried compacted samples. The dimenstons of individual PSD specimens were approximately $12 \times 8 \times 8 \mathrm{~mm}$. After trimming, the PSD specimens were placed in an oven for ten minutes in order to get rid of moisture which may have been absorbed by the specimens during trimming. The PSD specimens were then kept in glass bottles and placed in the desiccator.

For Soils $B$ and $C$, after permeability testing, the PSD specimens were carefully trimmed with a razor blade into cubes approxin.ately $8 \mathrm{~mm}$ on a side. The method for drying Soils $B$ and $C$ in this study was the freeze drying technique. The method consists of quickly freezing a small soil specimen to cryogenic temperatures, followed by vacuum drying the frozen sample to remove water by sublimation. This process eliminates the air-water menisci shrinkage effect, which may alter the soll structure, by replacing it with an air-solid system. The small shrinkage that does occur (1ess than $5 \%$ as determined by testing a few specimens initially) after freeze-drying indicates that minimal sample disturbance probably occurred.

The freeze-drying apparatus and procedures used in this study followed Garcia-Bengochea (1978). Appendix A-1 provides details of this apparatus and procedures.

After the specimens were dried, the PSD tests were conducted. The apparatus and procedures used in this study was similar to those used by Garcia-Bengochea (1978) and White (1980). Appendix A-2 provides detalls of PSD testing equipment and step by step procedures. However, 1t is worthwhile to point out here that both penetrometers (with Intrusion capacity of $0.2 \mathrm{cc}$ and $1.2 \mathrm{cc}$ respectively) were used in 
the present study. This is because the small penetrometer (with capacity $0.2 \mathrm{cc}$ ), as used by Garcia-Bengochea (1978) and White (1980), cannot properly measure the PSD of the high permeability soils A and B. Also note that the large penetrometer (with capacity $1.2 \mathrm{cc}$ ) can not be placed in the Amico porosimeter; therefore it can only go through low pressure intrusion which measures the distribution of the large size pores $(20 \sim 500 \mu \mathrm{m})$. For these reasons, a combination of data measured by both penetrometers is used to show the distribution of the entire range of pore sizes. 
CHAPTER, IV PRESENTATION AND DISCUSSION

\subsection{COMPACTION}

\subsubsection{Sample Nomenclature}

Each sample tested in this study was assigned a code name based on the soil type, compactive effort, compaction method, and relative water content with respect to its optimum. This code designation is listed in Table 4-1.

4.1.2 Dry Density versus Water Content Relationships for Soils $B$ and $C$.

The dry density versus water content relationships utilized in this study were determined from the standard and modified Proctor compaction tests. The specifications for these impact compaction tests are listed in Table 4-2. The Proctor test results are listed in Table 4-3. The statistical relationships between dry density and water content obtained from a least squares analysis of these data are shown in Figure 4-1. Satisfactory fit to the data is indicated by the high coefficients of multiple determination 1isted in Table 4-3.

A California type kneading compactor was used to compact most of the samples tested (i.e., Soils B and C) in this study. The kneading compaction dry density and water content points used in the testing program are shown in Figure 4-1. The gauge pressures utilized for each of these points are listed in Table 4-4. For Soil C, both results of high and medium compactive efforts are presented. However, 
TABLE 4-1 Sample Code Designation

Soil Type

$$
\begin{aligned}
& 0-\operatorname{Sol1} \text { A (Ottawa sand only) } \\
& \text { SC1 Sol1 B ( } 10 \% \text { Ottawa sand }+10 \% \text { Kaolin) } \\
& \text { SC3 Sol1 C ( } 30 \% \text { Ottawa sand }+30 \% \text { Kaolin) }
\end{aligned}
$$

Compactive Effort

For Sol1 A:

L - Relatively low effort

H - Relatively high effort

For Soils $B$ and $C$ :

M - Medium effort (specified in ASTM D698)

$\mathrm{H}$ - High effort (specified in ASTM D1557)

Water Content

o - Optimum water content

O-D - Very slightly dry of optimum

D Dry of optimum

W-Wet of optimum

Compaction Method

$$
\begin{aligned}
& \text { nil Kneading compaction } \\
& \text { P - Pluvial compaction }
\end{aligned}
$$

Example:

SC1M0: Soil B ( $90 \%$ Ottawa sand $+10 \%$ Kaolin), compacted by medium effort at optimum water content by kneading compaction. 


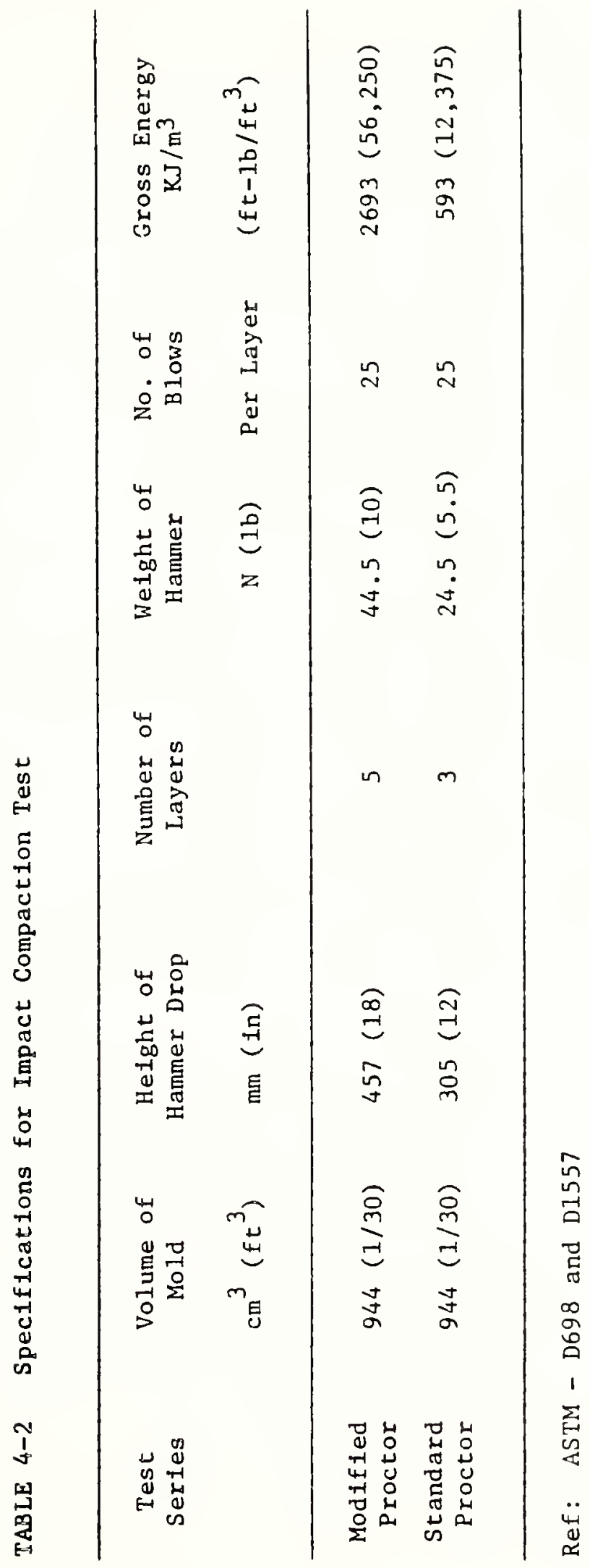




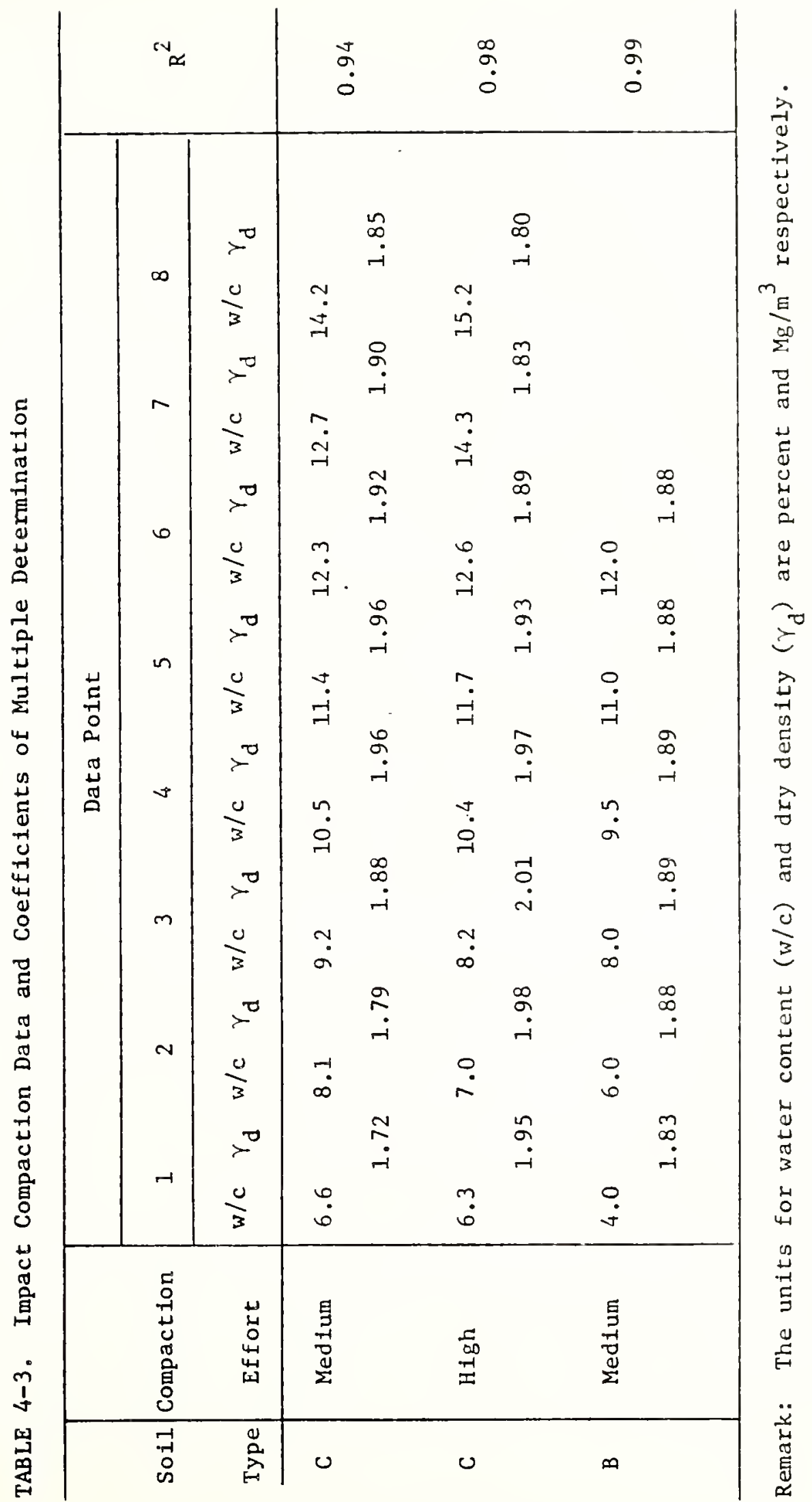




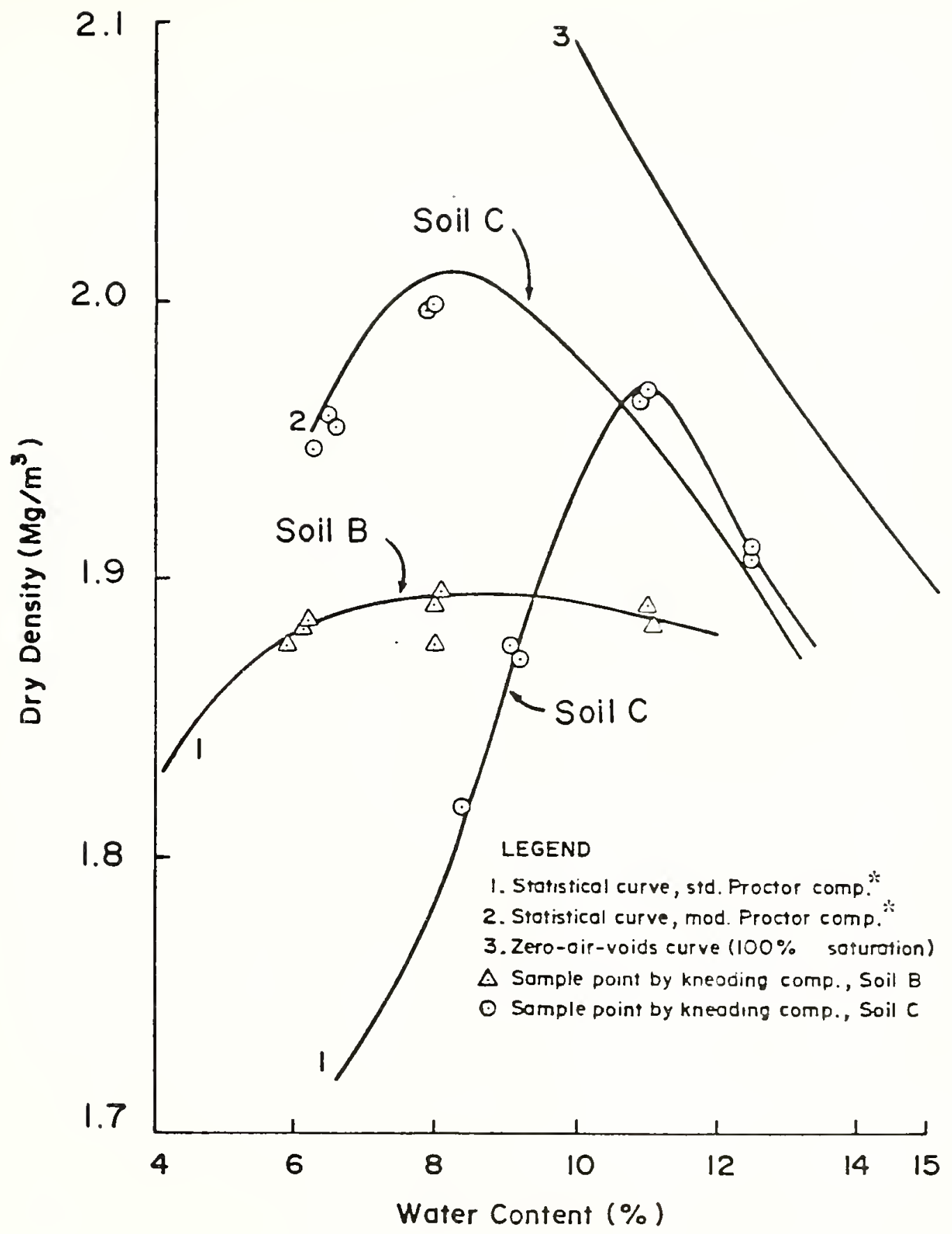

Figure 4-1 Dry Density versus Water Content Curves for Soils $B$ and $C$

\footnotetext{
*

Curves are somewhat displaced and different in shape from what would have been expected, but are based upon real data.
} 
TABLE 4-4 Kneading Compaction Varlables for Each Sample Point of Soils B and C

\begin{tabular}{|c|c|c|c|c|}
\hline $\begin{array}{l}\text { Sample } \\
\text { Code }\end{array}$ & $\begin{array}{l}\text { Sample } \\
\text { Point }\end{array}$ & $\begin{array}{l}\text { Water Content } \\
(\%)\end{array}$ & $\begin{array}{c}\text { Gauge Pressure } \\
\mathrm{kPa} \text { (psi) }\end{array}$ & $\begin{array}{l}\text { Achieved } \\
\text { Dry Density } \\
\left(\mathrm{Mg} / \mathrm{m}^{3}\right)\end{array}$ \\
\hline \multirow{3}{*}{$S C 3 M D$} & 1 & 9.1 & $55.2(8)$ & 1.87 \\
\hline & 2 & 9.2 & 55.2 & 1.87 \\
\hline & 3 & 8.4 & 55.2 & 1.81 \\
\hline \multirow{2}{*}{ SC3MO } & 1 & 11.0 & 55.2 & 1.96 \\
\hline & 2 & 10.9 & 55.2 & 1.96 \\
\hline \multirow{2}{*}{ SC $3 M W$} & 1 & 12.5 & 55.2 & 1.90 \\
\hline & 2 & 12.5 & 55.2 & 1.91 \\
\hline \multirow{3}{*}{ SC3HD } & 1 & 6.5 & $193.2(28)$ & 1.95 \\
\hline & 2 & 6.6 & 193.2 & 1.95 \\
\hline & 3 & 6.2 & 193.2 & 1.94 \\
\hline \multirow{2}{*}{ SC3HO-D } & 1 & 8.0 & $207.0(30)$ & 1.99 \\
\hline & 2 & 7.9 & 207.0 & 1.99 \\
\hline \multirow{3}{*}{$S C 1 M D$} & 1 & 6.2 & $34.5(5)$ & 1.88 \\
\hline & 2 & 6.1 & 34.5 & 1.88 \\
\hline & 3 & 5.9 & 34.5 & 1.87 \\
\hline \multirow{3}{*}{ SC1MO } & 1 & 8.0 & $41.4(6)$ & 1.89 \\
\hline & 2 & 8.1 & 41.4 & 1.89 \\
\hline & 3 & 8.0 & 41.4 & 1.87 \\
\hline \multirow{2}{*}{ SC1MW } & 1 & 11.0 & 34.5 & 1.89 \\
\hline & 2 & 11.1 & 34.5 & 1.88 \\
\hline
\end{tabular}


for Soll B, only the result of medium compactive effect of this soll was presented because 1) the compaction result was little affected by the magnitude of the impact compaction effort; and 2) the approprlate gauge pressures necessary to fit the results of kneading compaction to the desired density as defined by the modified Proctor compaction curve were not able to be found in the present study:

\subsubsection{Pluvial Compaction Results for Soil A}

The results of Soil A obtained from the pluvial compaction are listed in Table 4-5. The results indicate that the desired density can be replicated by controlling the diameter of hole and the height of drop.

\subsection{PERMEABILITY}

The permeability was measured by a falling head apparatus under a back pressure of $345 \mathrm{kPa}$ (50 psi). The hydraulic gradient during the tests ranged between 2 to 5 . The permeabilities were calculated from the usual falling head equation (Taylor, 1948)

$$
\mathrm{k}=\frac{\mathrm{aL}}{\Delta \mathrm{tA}} \ln \frac{\mathrm{h}_{\mathrm{o}}}{\mathrm{h}_{\mathrm{t}}}
$$

$$
\text { where } \begin{aligned}
\mathrm{k} & =\text { permeability } \\
\mathrm{a} & =\text { cross-sectional area of the standpipes } \\
\mathrm{A} & =\text { cross-sectional area of sample } \\
\Delta t & =\text { time increment } \\
\mathrm{h}_{0} & =\text { total head at zero time } \\
\mathrm{h}_{\mathrm{t}} & =\text { total head after time } \Lambda \mathrm{t} .
\end{aligned}
$$

Figure 4-2 shows a schematic diagram of the falling head test. 
TABLE 4-5 Pluvial Compaction Variables for Each Sample Point of Soil A

\begin{tabular}{|c|c|c|c|c|c|}
\hline $\begin{array}{l}\text { Sample } \\
\text { Code }\end{array}$ & $\begin{array}{l}\text { Compacted } \\
\text { Sample } \\
\text { Point }\end{array}$ & $\begin{array}{c}\text { Diameter of } \\
\text { Hole }(\mathrm{mm})\end{array}$ & $\begin{array}{l}\text { Height of } \\
\text { Drop (nm) }\end{array}$ & $\begin{array}{l}\text { Number } \\
\text { of Layers }\end{array}$ & $\begin{array}{c}\text { Achieved } \\
\text { Dry Density } \\
\left(\mathrm{Mg} / \mathrm{m}^{3}\right)\end{array}$ \\
\hline \multirow{6}{*}{ OPL } & 1 & 6.8 & 108 & 5 & 1.62 \\
\hline & 2 & 6.8 & 108 & 5 & 1.62 \\
\hline & 3 & 6.8 & 108 & 5 & 1.62 \\
\hline & 4 & 6.8 & 108 & 5 & 1.62 \\
\hline & 5 & 6.8 & 108 & 5 & 1.62 \\
\hline & 6 & 6.8 & 108 & 5 & 1.62 \\
\hline \multirow{6}{*}{$\mathrm{OPH}$} & 1 & 3.8 & 108 & 5 & 1.72 \\
\hline & 2 & 3.8 & 108 & 5 & 1.72 \\
\hline & 3 & 3.8 & 108 & 5 & 1.72 \\
\hline & 4 & 3.8 & 108 & 5 & 1.72 \\
\hline & 5 & 3.8 & 108 & 5 & 1.72 \\
\hline & 6 & 3.8 & 108 & 5 & 1.72 \\
\hline
\end{tabular}




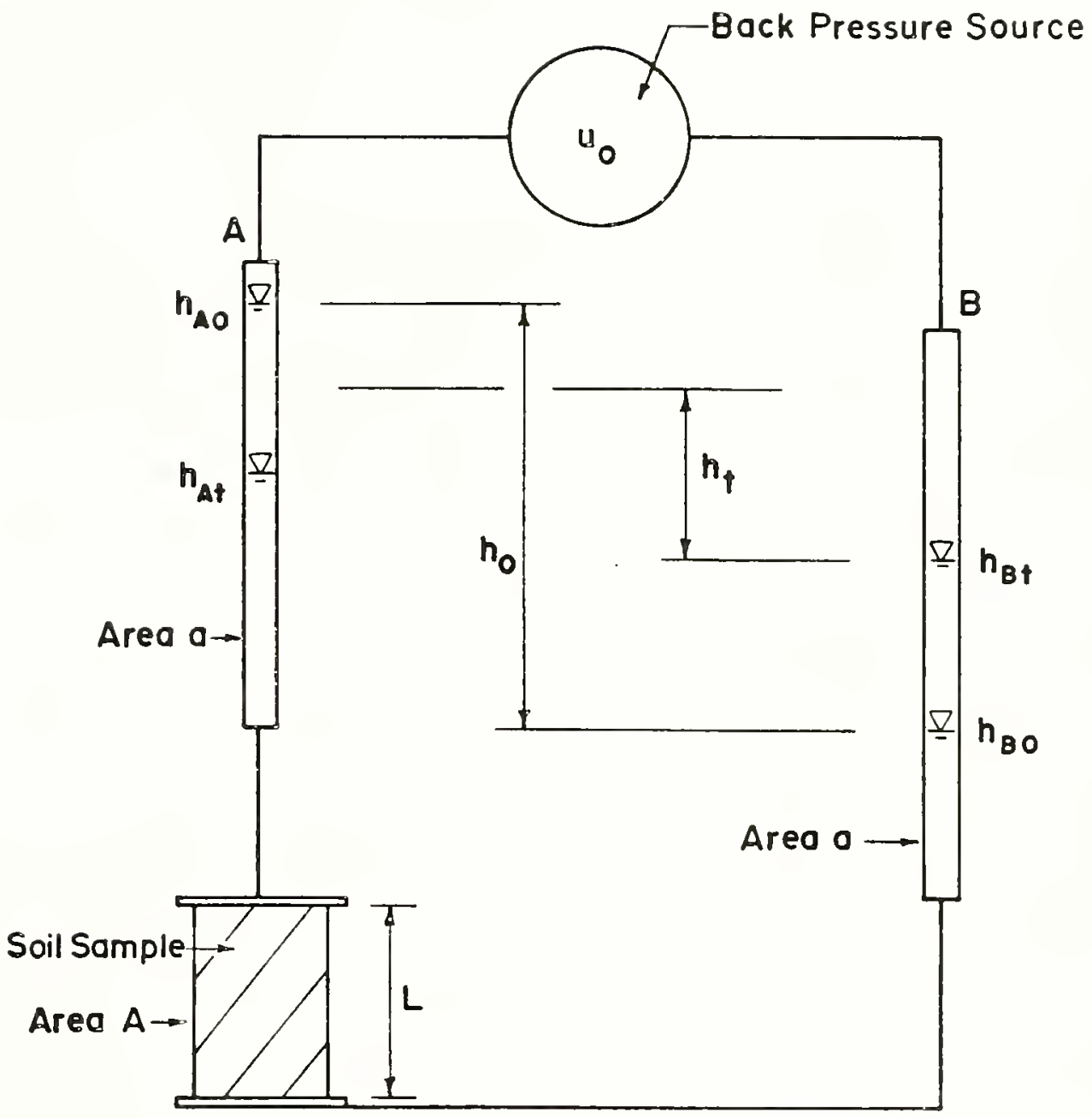

Figure 4-2 Schematic Diagram of Falling Head Permeability Test (After Garcia-Bengochea, 1978) 
It is evident from the figure that

$$
\begin{aligned}
h_{0} & =\left(h_{A O}+u_{0}\right)-\left(h_{B O}+u_{O}\right) \\
& =h_{A O}-h_{B O}
\end{aligned}
$$

where $u_{0}=$ back pressure on the system

and

$$
h_{t}=h_{A t}-h_{B t}
$$

The permeability is then calculated from Equation (4-1).

The variation of the room temperature during permeation of most of the permeability tests was nagligible $\left(\mathrm{e} . \mathrm{g} ., \pm 0.4{ }^{\mathrm{C}} \mathrm{C}\right)$, although in a few tests the variation was up to $\pm 1^{\circ} \mathrm{C}$ or so. For each test, an average temperature was taken and the following equation suggested by Taylor (1948) was used to calculate and to report the permeability:

$$
\begin{aligned}
\mathrm{k}_{20}{ }^{\circ} \mathrm{C} & =\mathrm{k}_{\mathrm{T}} \cdot \frac{\mu_{\mathrm{T}}}{\mu_{20}{ }^{\circ} \mathrm{C}} \\
\text { where } \mathrm{k}_{20}{ }^{\circ} \mathrm{C} & =\text { permeability at temperature } 20^{\circ} \mathrm{C} \\
\mathrm{k}_{\mathrm{T}} & =\text { permeability at temperature } \mathrm{T} \text { of test } \\
\mu_{\mathrm{T}} & =\text { viscosity of water at temperature } \mathrm{T} \\
\mu_{20}{ }^{\circ} \mathrm{C} & =\text { viscosity of water at temperature } 20^{\circ} \mathrm{C} .
\end{aligned}
$$

A summary of the permeability test results of the compacted samples 1 isted in Table 4-4 and Table $4-5$ is given in Table $4-6$. In this table the permeability is normalized to an ASTM standard temperature of $20^{\circ} \mathrm{C}$

For Soil C, the dry-side samples had permeabilitics une to two orders of magnitude greater than the optimum and wet-side samples. 
TABLE 4-6 Permeabilities of Samples Tested

\begin{tabular}{|c|c|c|c|c|}
\hline $\begin{array}{l}\text { Soil } \\
\text { Type }\end{array}$ & $\begin{array}{l}\text { Sample } \\
\text { Code }\end{array}$ & $\begin{array}{l}\text { Point } \\
\text { No. }\end{array}$ & $\begin{array}{c}\mathrm{k}_{20}{ }^{\circ} \mathrm{C} \\
(\mathrm{cm} / \mathrm{sec})\end{array}$ & $\begin{array}{c}\text { Average } \mathrm{k}_{20}{ }^{\circ} \mathrm{C} \\
(\mathrm{cm} / \mathrm{sec})\end{array}$ \\
\hline \multirow{5}{*}{$\mathrm{C}$} & SC3MD & $\begin{array}{l}1 \\
2 \\
3\end{array}$ & $\begin{array}{l}4.6 \times 10^{-5} \\
4.6 \times 10^{-5} \\
4.2 \times 10^{-5}\end{array}$ & $4.5 \times 10^{-5}$ \\
\hline & SC3MO & $\begin{array}{l}1 \\
2\end{array}$ & $\begin{array}{l}7.1 \times 10^{-7} \\
7.5 \times 0^{-7}\end{array}$ & $7.3 \times 10^{-7}$ \\
\hline & SC3MW & $\begin{array}{l}1 \\
2\end{array}$ & $\begin{array}{l}6.1 \times 10^{-7} \\
5.8 \times 10^{-7}\end{array}$ & $6.0 \times 10^{-7}$ \\
\hline & SC $3 H D$ & $\begin{array}{l}1 \\
2 \\
3\end{array}$ & $\begin{array}{l}1.8 \times 10^{-6} \\
1.7 \times 10^{-6} \\
2.1 \times 10^{-6}\end{array}$ & $1.9 \times 10^{-6}$ \\
\hline & SC3HO-D & $\begin{array}{l}1 \\
2\end{array}$ & $\begin{array}{l}6.1 \times 10^{-7} \\
6.0 \times 10^{-7}\end{array}$ & $6.1 \times 10^{-7}$ \\
\hline \multirow{3}{*}{ B } & SCIMD & $\begin{array}{l}1 \\
2 \\
3\end{array}$ & $\begin{array}{l}3.6 \times 10^{-3} \\
3.0 \times 10^{-3} \\
3.9 \times 10^{-3}\end{array}$ & $3.5 \times 10^{-3}$ \\
\hline & SCIMO & $\begin{array}{l}1 \\
2 \\
3\end{array}$ & $\begin{array}{l}1.3 \times 10^{-3} \\
1.3 \times 10^{-3} \\
1.2 \times 10^{-3}\end{array}$ & $1.3 \times 10^{-3}$ \\
\hline & SCIMW & $\begin{array}{l}1 \\
2\end{array}$ & $\begin{array}{l}5.6 \times 10^{-4} \\
5.0 \times 10^{-4}\end{array}$ & $5.3 \times 10^{-4}$ \\
\hline A & OPL & $\begin{array}{l}5 \\
6 \\
5 \\
6\end{array}$ & $\begin{array}{l}2.7 \times 10^{-2} \\
2.3 \times 10^{-2} \\
1.4 \times 10^{-2} \\
1.3 \times 10^{-2}\end{array}$ & $1.4 \times 10^{-2}$ \\
\hline
\end{tabular}


There was 1ittle difference between the permeabl11ties of the opt1mum and wet-side samples. These trends were found by the prevfous researchers, e.g., Lambe (1954), Mitche11, Hooper and Campanella (1965), and Garcia-Bengochea (1978). However, for Soil B which contains only $10 \%$ clay by weight, the above trend is not very clear; i.e., the difference between the dry-side and optimum samples was small. Two possible reasons are 1 ) water content is not a sensitive variable in the compaction of this soil, as indicated in Figure 4-1, and 2) the dry-side samples tested are not sufficie:tly dry of optimum (see Figure 4-1). Strictly speaking, there is no clear optimum water content on the standard Proctor compaction curve of this soil. This may be the nature of this poorly graded sandy soil.

\subsection{PORE SIZE DISTRIBUTION}

\subsubsection{Data Reduction}

As mentioned in Section 2.1.1, the absolute applied pressures can be converted to apparent intruded pore diameters based on Equation (2-1). This step requires that the surface tension and contact angle be known. The surface tension used in this study is assumed to be 484 dynes/cm for all soils used (Kemba11, 1946 and Bhasin, 1975). The contact angle of mercury on Kaolin is $147^{\circ}$ (Diamond, 1970) and that of mercury on quartz (sand) is also $147^{\circ}$ (Ellison, Klemm and Schwartz, 1967). Based on these reported values (both at $25^{\circ} \mathrm{C}$ ), the contact angle of mercury on Soils $\mathrm{B}$ and $\mathrm{C}$ is assumed to be $147^{\circ}$. For Soil $\mathrm{A}$, the contact angle of mercury on phenolic resin was determined to be $154^{\circ}$ following the method suggested by Winslow $(1969,1980)$. Since the surface tension of phenolic resin is much lower than that of sand, therefore the con- 
tact angle of mercury on Soil A was assumed to be $154^{\circ}$.

For each PSD test, the applied pressures and intrusion data were recorded. The pressures recorded during the filling operation were absolute pressures, while those recorded durlng the porosimeter operation (called high pressure intrusion) were gage pressures. As noted by Bhasin (1975), a net correction of $76 \mathrm{kPa}$ (11 psi) accounting for atmospheric pressure and negative head of mercury is added to the gauge pressures to obtain the absolute pressures. The absolute pressures are next converted to apparent intruded pore diameters by Eqtation $(2-1)$. The next step in the calculation is to apply the corrections to the intrusion data. The corrections required during low pressure intrusion are to account for the fact that part of the intrusion recorded is actually the compression of the air that was trapped in the penetrometer during the mercury-filling period (refer to Appen$\operatorname{dix}(\mathrm{A}-2)$. The high pressure correction to the intrusion data is to arcount for the compressibility of the sample, penetrometer and mercury, as well as the temperature changes that occur as a result of pressurization. A "blank test" for this correction had been outlined in a proposed ASTM standard, subcommittee D18.14. In this study, a correction of $0.001 \mathrm{ml}$ per $\mathrm{ml}$ of mercury in the penetrometer at $103,500 \mathrm{kPa}$ (15,000 psi), as noted by Bhasin (1975), was applied.

A computer program developed by Reed (1977) was utilized to reduce the $\mathrm{PSD}$ data. This program consisted of the above calculations and corrections. A significant modification of this program has been made, especially for the graphical presentations of the PSD data and the finite difference approximation (see Section 4.3.2). A listing of this modified computer program and sample output are given 
In Appendix A-3.

\subsubsection{Data Presentation}

The PSD data are normally expressed in terms of the cumulative volume of pore space intruded as a function of pore diameter. Since a wide range (e.g., several orders of magnitude) of pore sizes is measured, it is often convenient to present pore diameter on a logarithmic scale. A graphical cumulative distribution with the intruded volumes per gram on the ordinate to an arithmetic scale and the limiting pore diameters ( called apparent pore diameters) on the abscissa to a logarithmic scale is a common way to present the PSD test results, e.g., Diamond (1970), Ahmed (1971), Bhasin (1975), Cebeci, et a1. (1978), and the proposed ASTM standard, subcomnittee D18.14. From Equation (2-5a) it is evident that the ordinate of the density function plot is $-d\left(V_{t}-V\right) / d x$. Multiplying this derivative quantity by $x / 2.303$ and rearranging gives $-d\left(V_{t}-V\right) / d(\log x)$. Since the cumulative distribution is expressed as $\left(V_{t}-V\right)$ versus $\log x$, it is preferred to plot the quantity $-d\left(V_{t}-V\right) / d(\log x)$ instead of $-d\left(V_{t}-V\right) / d x$ versus $\log x$. The density function value can be obtained by (1) directly taking the derivative of $F_{v x}(x)$ which is a curve-fitted function of the above cumulative pore size distribution curve, or (2) using a Einite difference approximation. The first method is obvious and its accuracy depends on goodness of curve-fitting. In this study, the pore size density function of soils is obtained in this manner (see Section 4.3.3). The second method was first used by Garcia-Bengochea (1978) for the graphical presentation of the PSD test data. However, since pore size is a continuous random variable, it is appropriate to present a pore 
size density function instead of a frequency polygon, which was used by Garcia-Bengochea. To approximate the density function a frequency histogram is plotted (i.e., $\Delta\left(V_{t}-V\right)$ versus $\log x$, see Figure 4-3a). For a good approximation, the class width, $\Delta(\log x)$, has to be an appropriate constant.

Assume a constant $c$ is chosen, then

$$
\begin{aligned}
& \log x_{i-1}-\log x_{i}=c \\
& \text { or } \frac{x_{i-1}}{x_{i}}=10^{c}
\end{aligned}
$$

From Equation (2-1), it is evident that the applied pressure is inversely proportional to the pore diameter. Therefore this approximation method requires that the applied pressure, $P$ be raised as follows:

$$
\frac{P_{i}}{P_{i-1}}=10^{c}
$$

Note that $\Delta\left(V_{t}-V\right)$ represents change in intruded pore volume, which is the difference between the two successive intrusions, and it can be calculated directly from intrusion record. The pore size density function plot is approximated by connecting the points of [ $\log$ $\left.d_{i}, f\left(\log d_{i}\right)\right]$ as shown in Figure 4-3b, where $\log d_{i}$, the midpoint of each class, is calculated as follows:

$$
\log d_{i}=\frac{1}{2}\left(\log x_{i-1}+\log x_{i}\right)
$$

or

$$
d_{i}=\sqrt{x_{i-1} \cdot x_{i}}
$$

and $f\left(\log d_{i}\right)$ is the probability density at $\log d_{i}$, which is calculated as follows: 


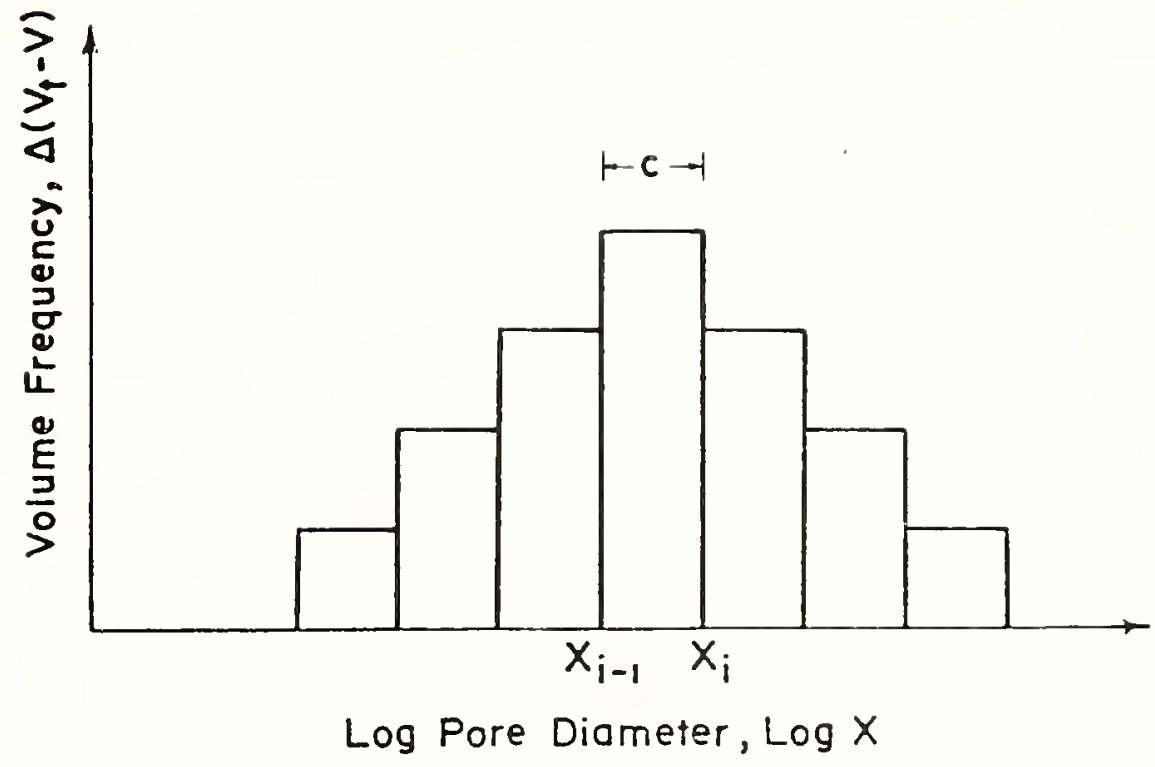

Figure 4-3(0) Frequency Hisłogram

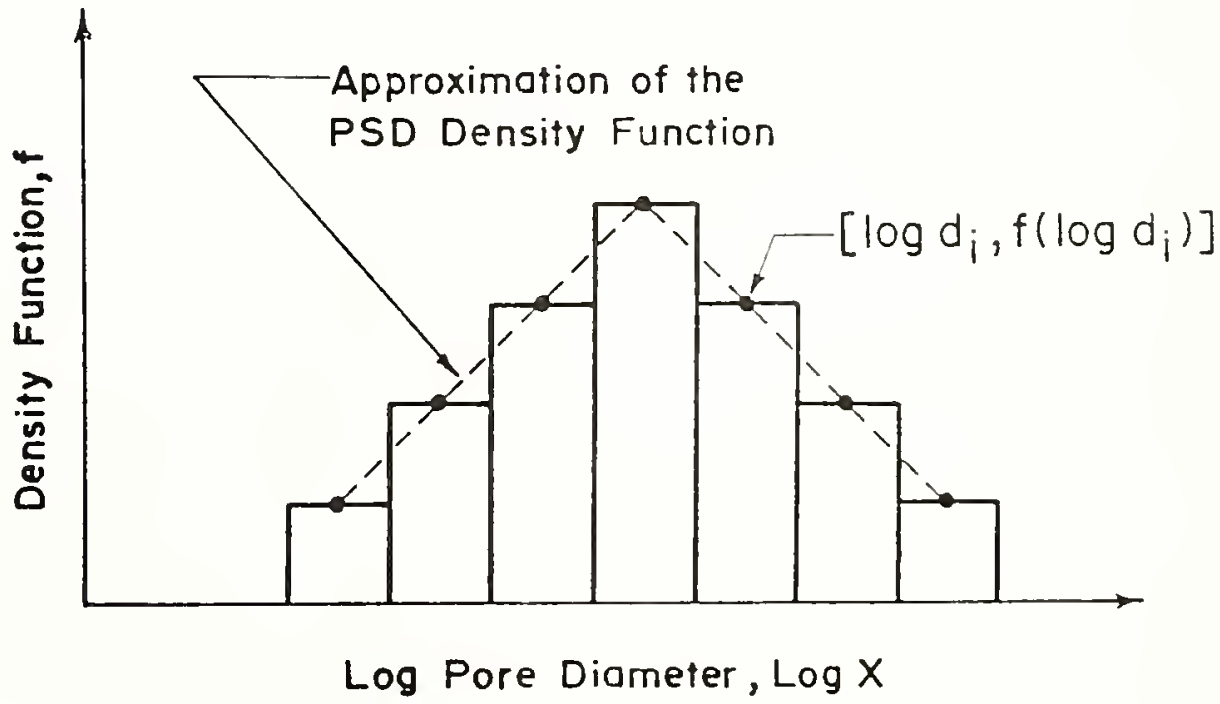

Figure 4-3(b) Finite Difference Approximation to PSD Density Function 


$$
f\left(\log d_{i}\right)=\frac{A\left(V_{t}-V\right)}{\Delta(\log x)}
$$

$$
=\frac{\left(V_{t}-V\right) \text { at } P_{i}-\left(V_{t}-V\right) \text { at } P_{i-1}}{\log x_{i-1}-\log x_{i}}(4-10)
$$

or

$$
f\left(\log d_{i}\right)=\Delta\left(v_{t}-v\right) / c
$$

A few specimens were tested and presented according to this finite difference approximation method. Figure 4-4 shows a comparison of the above two approaches. It is evident that these two methods match up very well if an appropriate constant $c$ is used. The value of $c$ should be as small as possible. However the appropriate value $P$ c depends on the pore size characteristics of soils. The wider the pore size range is the larger the value of $c$ should be. In this study, the value of $c$ varied from 0.1 to 0.2 .

In addition, some investigators (e.g., Lohnes, et al., 1976 and Diamond, et al., 1972) prefer to express pore size distribution as a dimensionless term (e.g., percentage) for the convenience of the mathematical treatment. Equations $(2-6)$ and $(2-7)$ provide the theoretical basis for this approach. If the pore size distribution data are to be correlated with soil properties, it is desirable to express the pore size distribution in mathematical forms. Hence it is convenient to express pore size distribution as a percentage. In Equation (2-7), the term $V_{t}$ represents the total void volume that can be intruded by mercury. According to this definition, the cumulative distribution for pores with size greater than a lower limit pore diameter (which depends on the capacity of porosimeter) should be $100 \%$ no matter how much unintruded pore space is left. On the other hand, if the term $V_{t}$ 

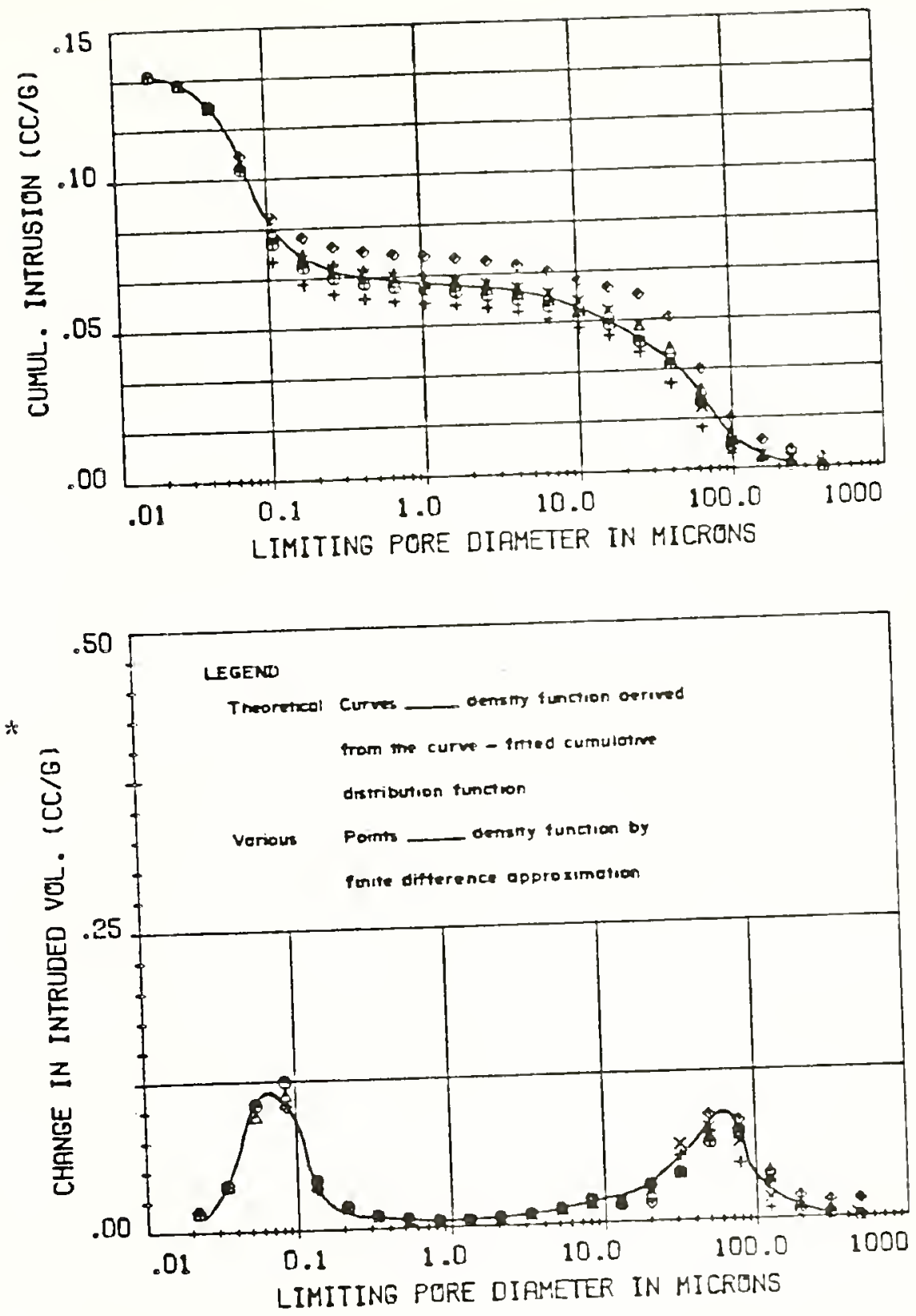

Figure 4-4 Comparison of Finite Difference Approximation Method and Direct Differentiation for Samples SC 3MD (Six PSD Data Sets)

The unit of density function is indeed ( $\mathrm{cc} / \mathrm{g} / \mathrm{chosen}$ diameter interval), but the chosen diameter interval is dimensionless (set Eq. 4-11). 
represents total void volume that exists in the sample, the cumulative distribution for pores with size greater than a lower limit pore diameter may not be $100 \%$, but depends on how much pore space is unintruded.

\subsubsection{Pore Size Distribution Curves and Fittings of the Curves}

Following the preceeding discussion of data presentation, the pore size distribution curves directly generated from the PSD data are shown in Appendix A-4. In addition to the cumulative distribution curves, some figures also show the density function curves whenever the finite difference approximation method is applicable.

At least three PSD specimens were tested for each compacted sample and two compacted samples were tested for each sample code. In general, the replication of the PSD tests was satisfactory. However some scatter is present in these data points. The reasons for scatter are:

1. Some of the specimens might have suffered more disturbance than others during trimming and transportation of the specimens. This is especially a problem for Soil B in which insufficient cohesion is created by the small amount of clay (1.e., 10\%).

2. The compacted samples themselves might not be completely homogeneous.

3. Measurement errors may also account for scatter in replicate pore size distribution curves, especially when a trapped air pocket on the surface of the specimen happens at the filling stage (see Appendix A-2) or when the pore volume of a specimen exceeds the intrusion capacity of the porosimeter.

4. The variability of replicate compacted samples may also account Eor the scatter shown in these figures. 
A plecewise cubic function based on the least squares method was selected to fit the 2SD curve. A computer program called "SPLIME" in the Purdue University $\operatorname{CDC}$ 6500-6600 computer library was used to do the least squares analysis. A description of "SPLINI" and some sample output are listed in Appendix A-5. The coefficients of the piecewise cubic functions for all samples are also listed in this Appendix.

The next step is to calculate the functional values of the pore size distribution and its probability density and then to present the graphical results of the PSD tests. Figure 4-5 shows a flow chart of the procedures. A computer program for this calculation is listed in Appendix A-6. Figure 4-6 through Figure 4-15 show these graphical results. Each of the figures consists of a cumulative distribution and its density function with the volume for gram on the ordinate to an arithmetic scale and the limiting pore diameter (or called apparent pore diameter) on the abscissa to a logrithmic scale. The density function provides a useful complement to the cumulative distribution curve for representing pore size characteristics. The pore size density function curves displayed bimodal characteristics for clay-sand mixtures (i.e., Soils B and C). This was the case found by Garcia-Bengochea (1978) for clay-silt mixtures. However, a single mode characteristic was found for Soil A. This is logical since Soil A contains only sand grains.

For the bimodal characteristics of Soils B and $C$, the small pore mode occurred consistently around $0.1 \mu \mathrm{m}$ and the large pore mode was between 10 and $100 \mu \mathrm{m}$. For the single modal characteristic of Soil A, the large pore mode occurred around $200 \mu \mathrm{m}$. It is obvious that the mode and its probability density may serve as useful qualitative 


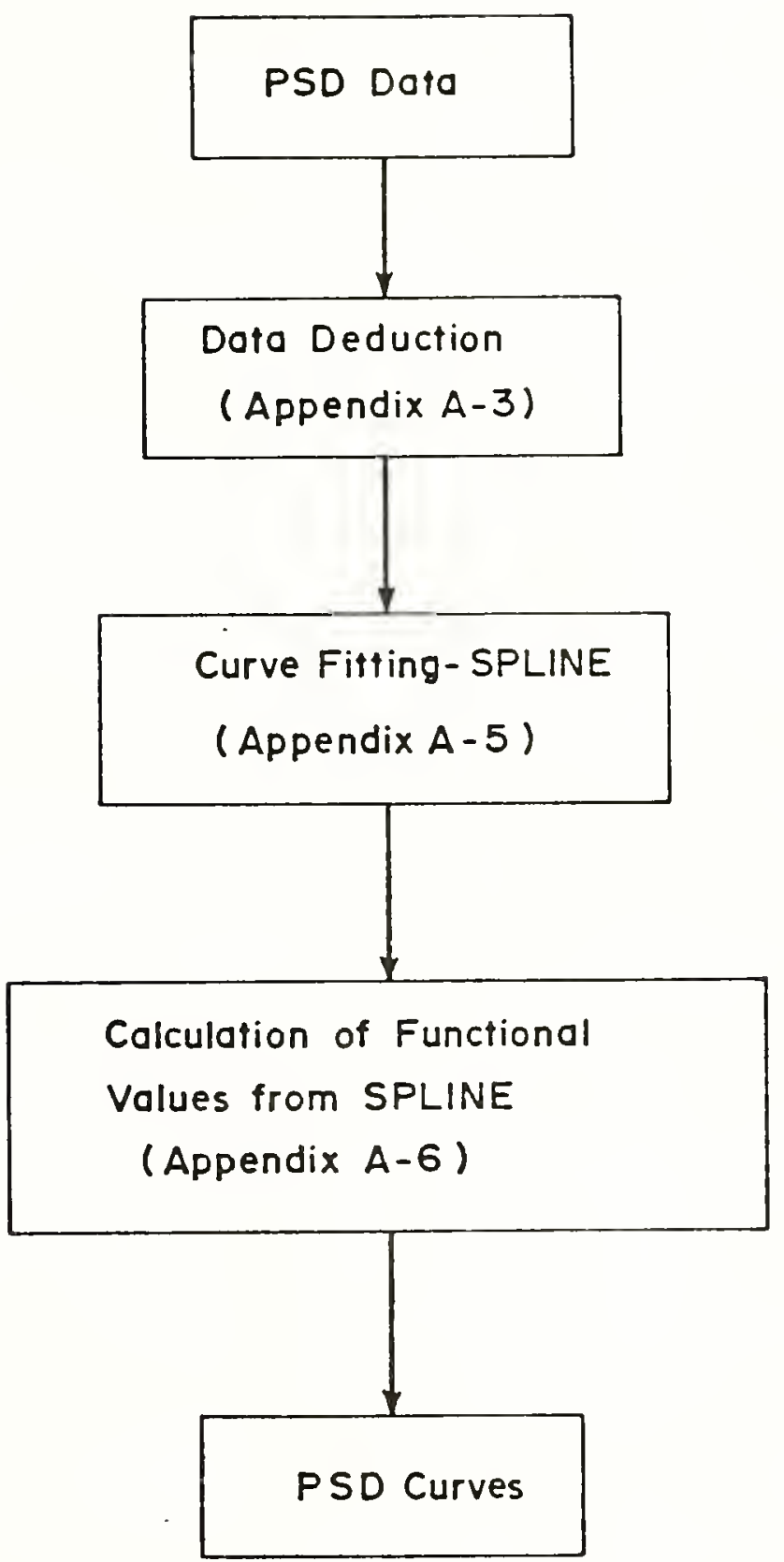

Figure 4-5 Flow Chart of Procedures for the PSD Curves 

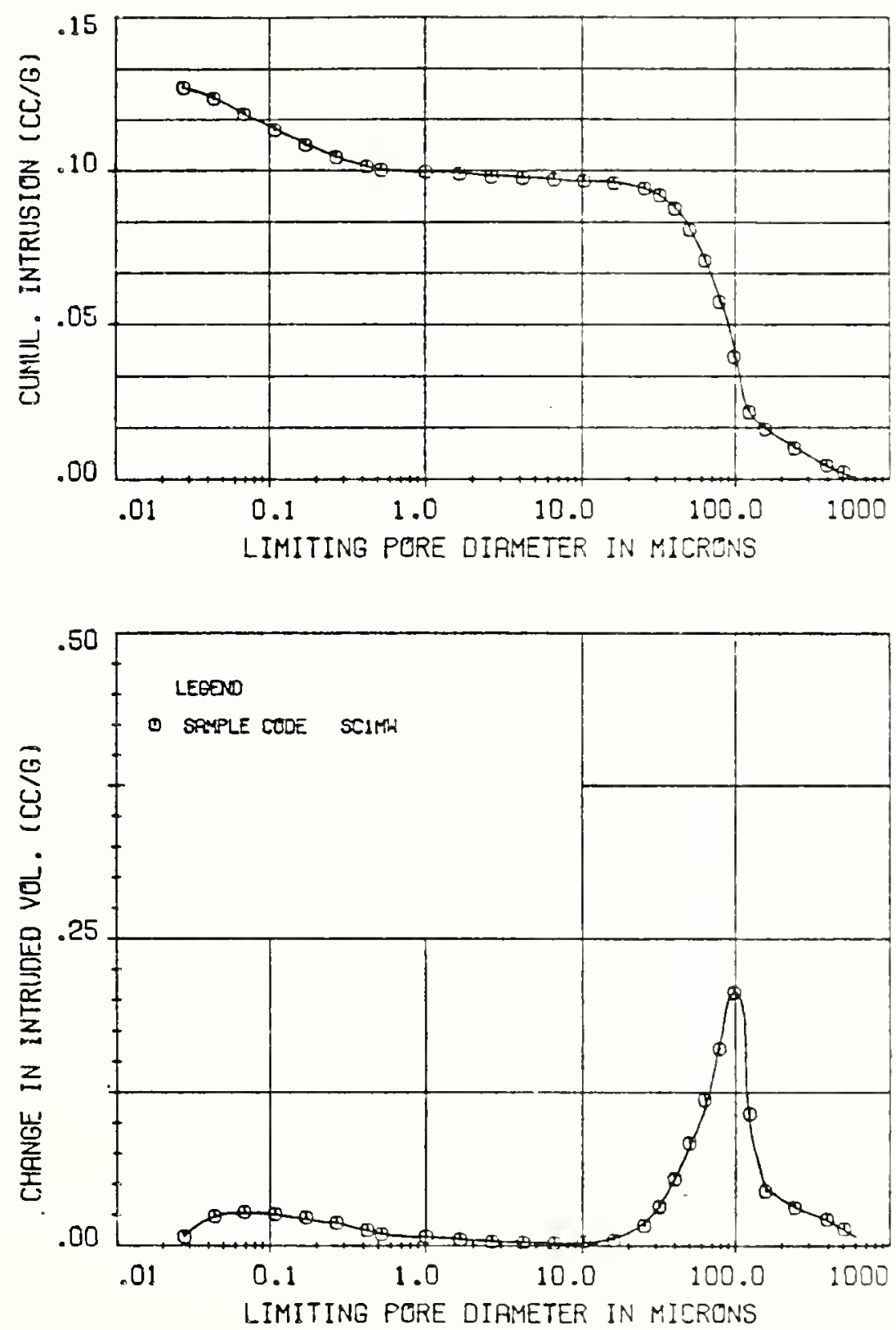

Figure 4-6 PSD Curves of SCIMW 

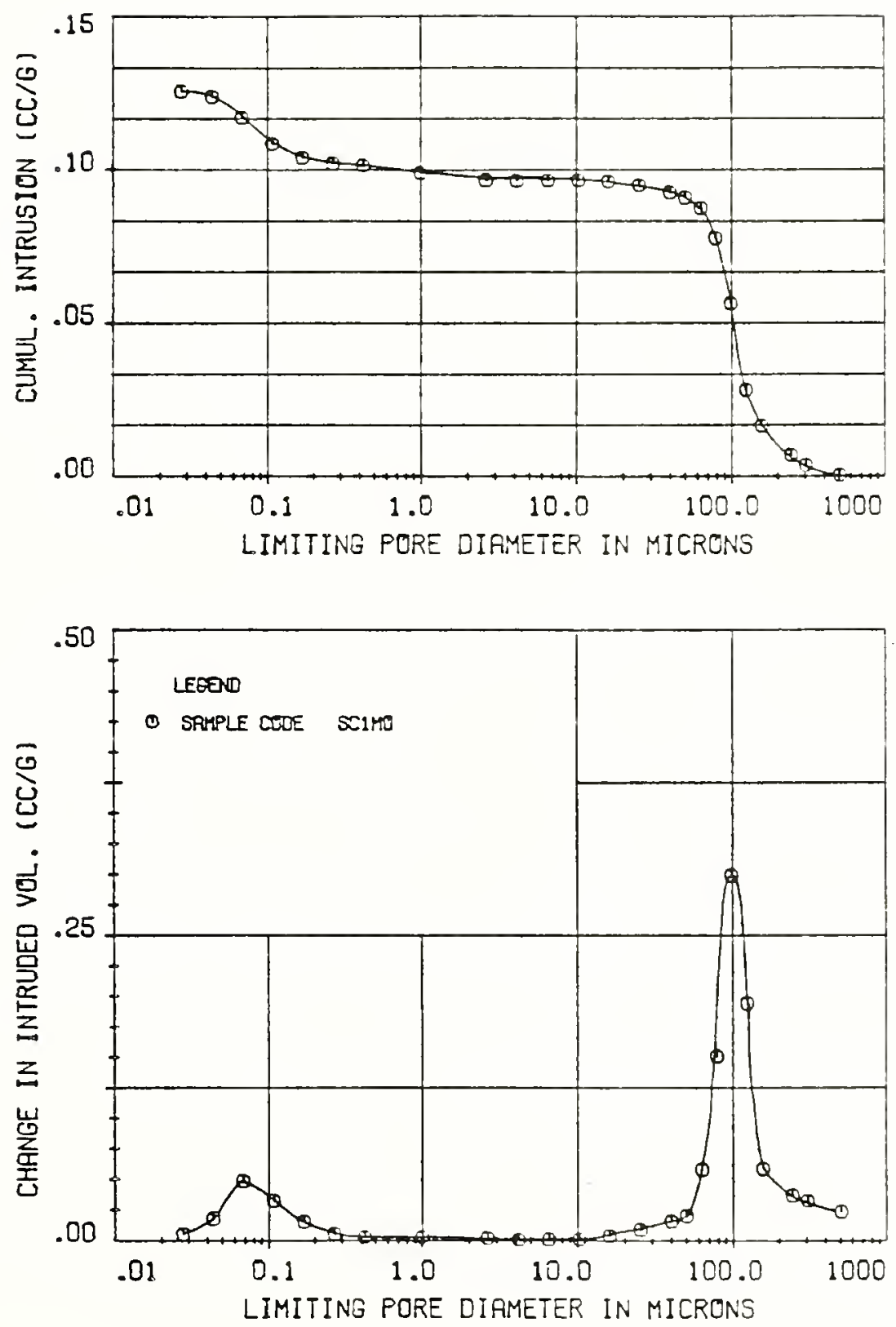

Figure 4-7 PSD Curves of SCIMO 

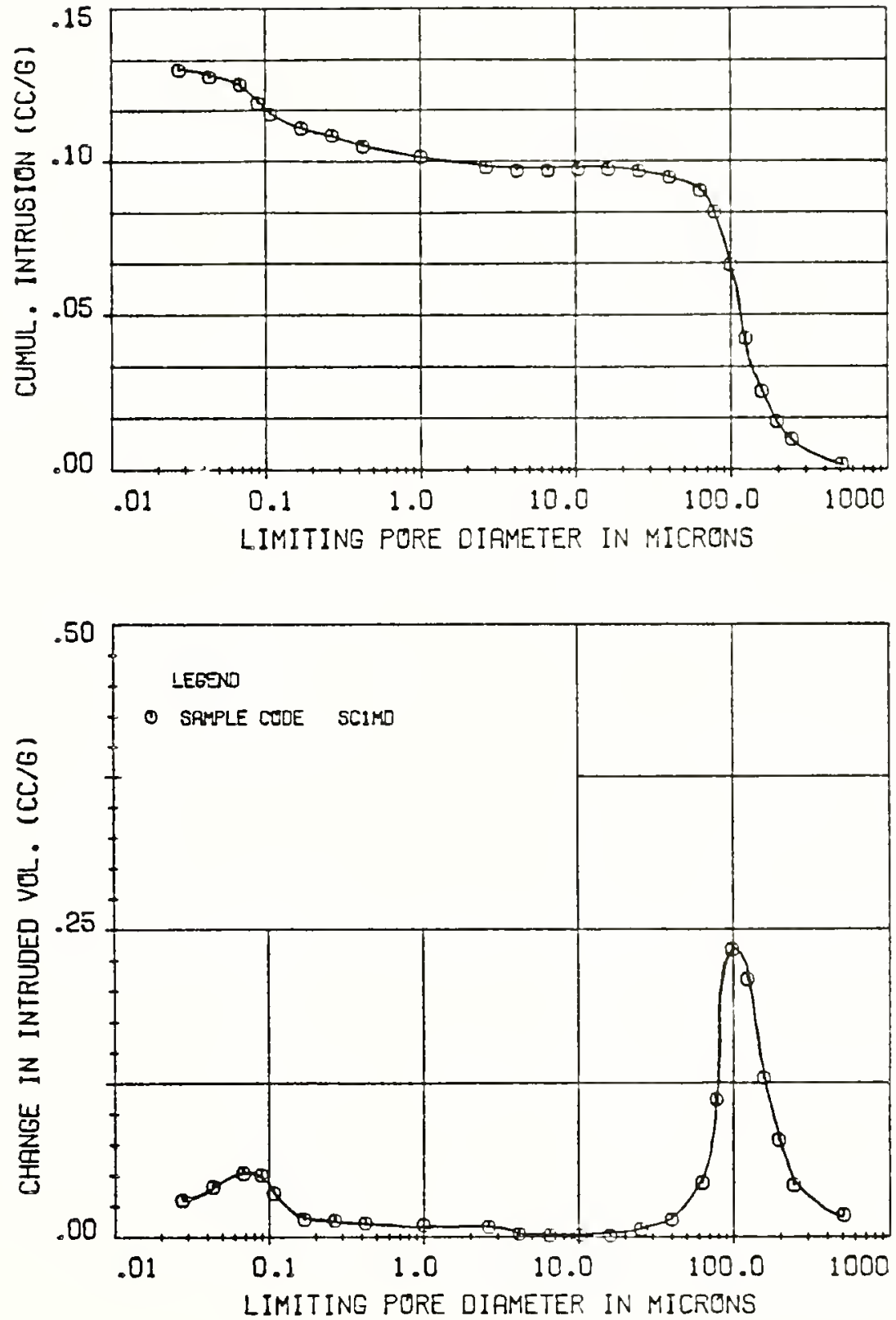

Figure 4-8 PSD Curves of SCIMD 

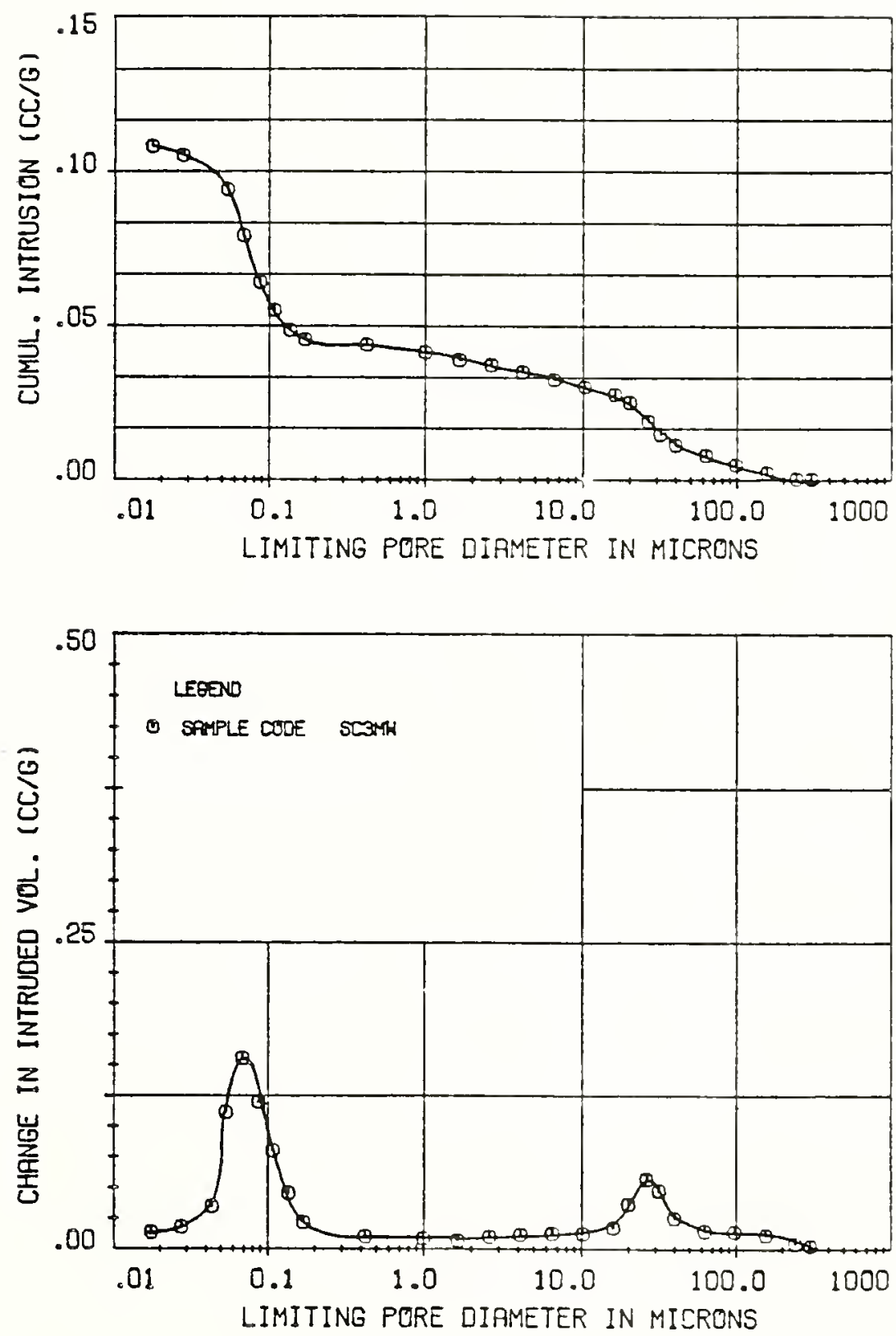

Figure 4-9 PSD Curves of SC3MW 

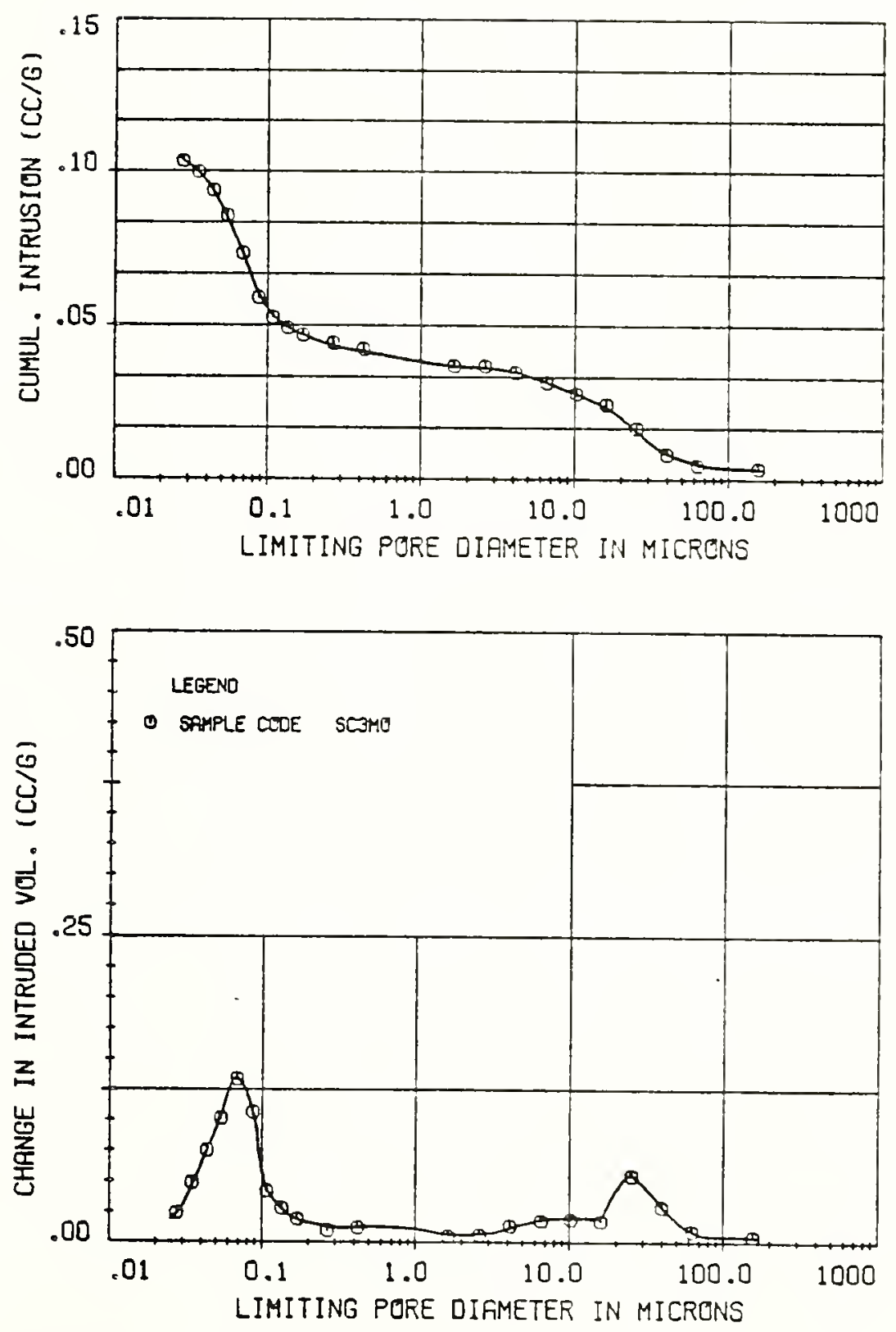

Figure 4-10 PSD Curves of SC3MO 

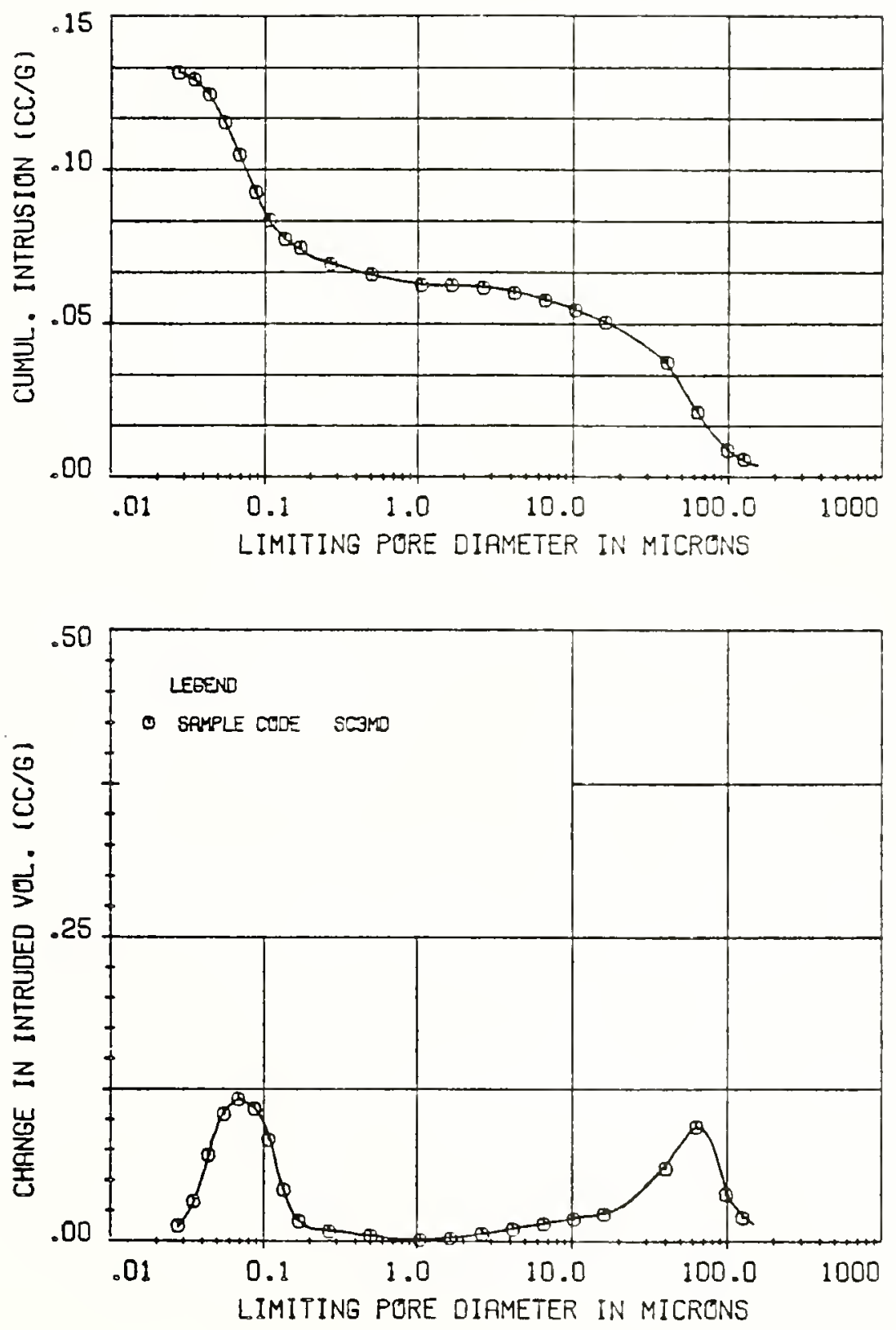

Figure 4-11 PSD Curves of SC3MD 

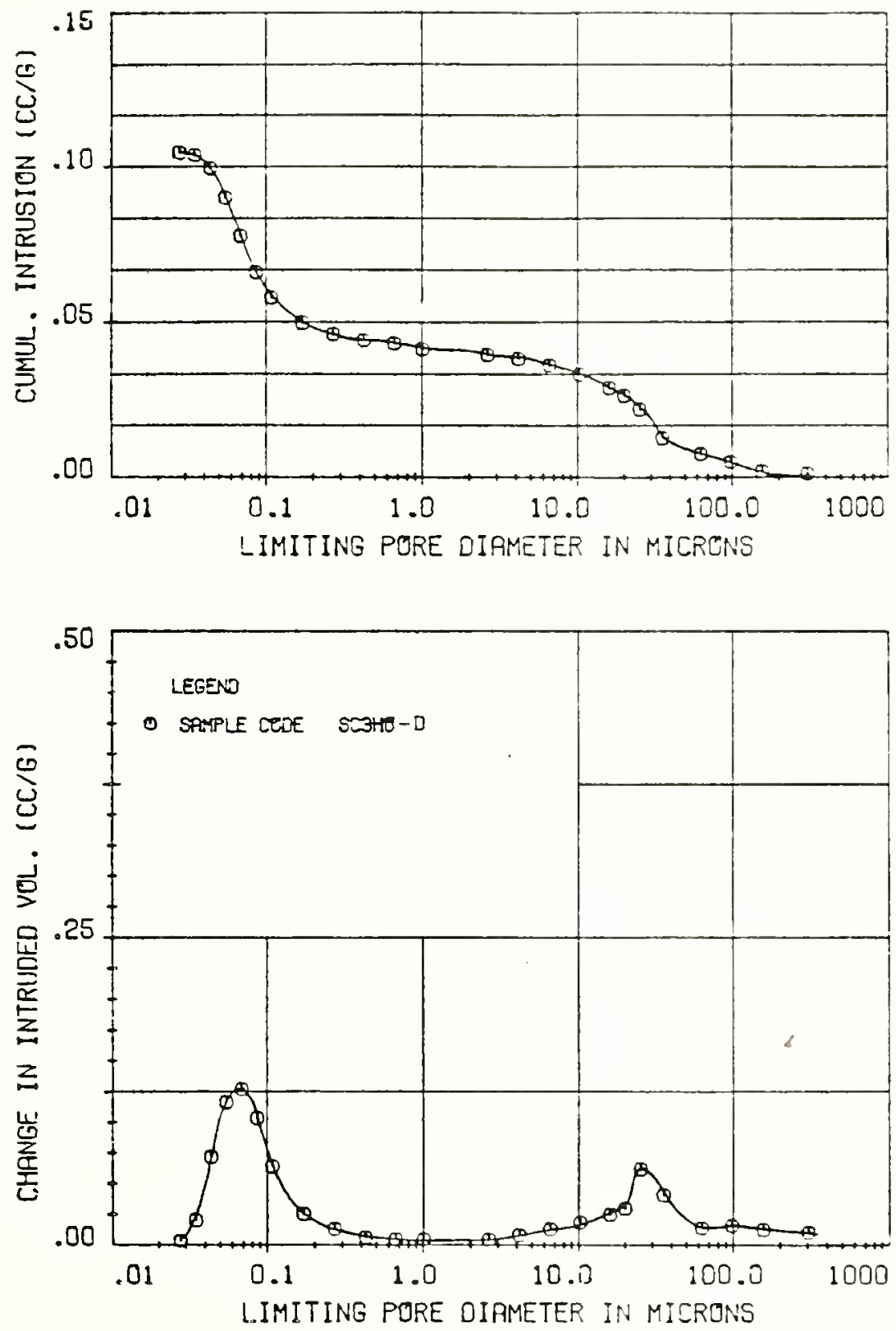

Figure 4-12. PSD Curves of SC3HO-D 

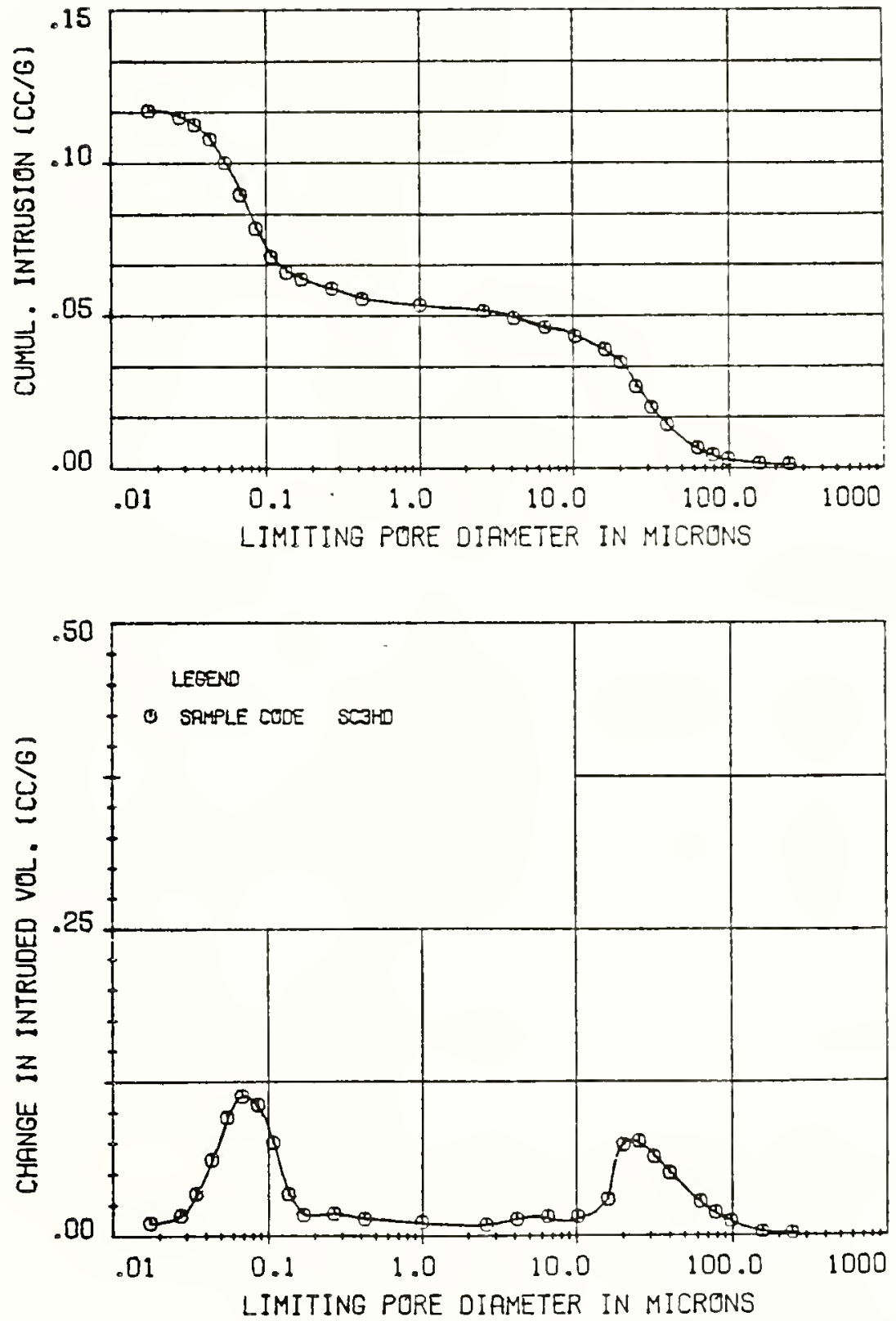

Figure 4-13 PSD Curves of SC3HD 

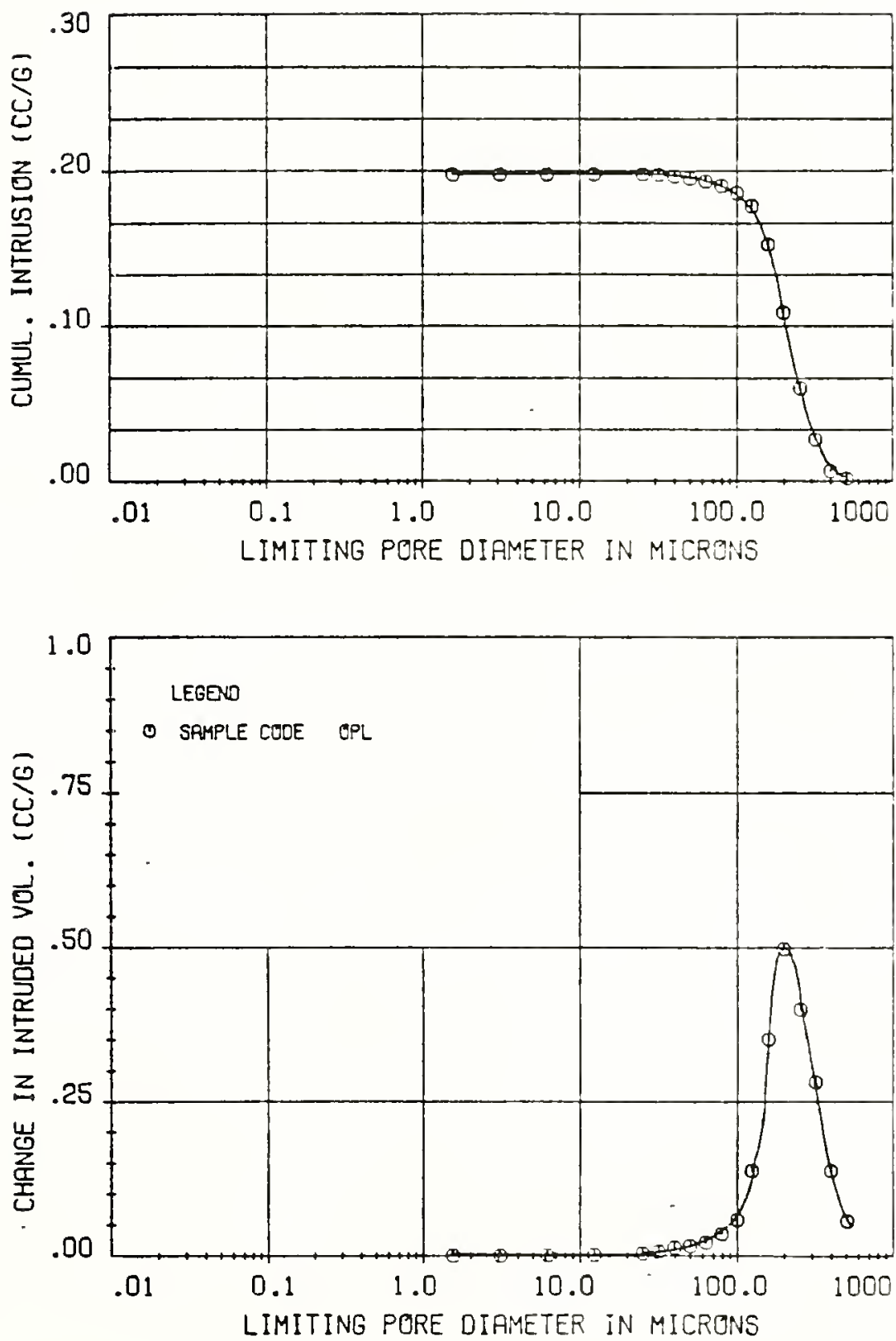

Figure 4-14 PSD Curves of OPL 

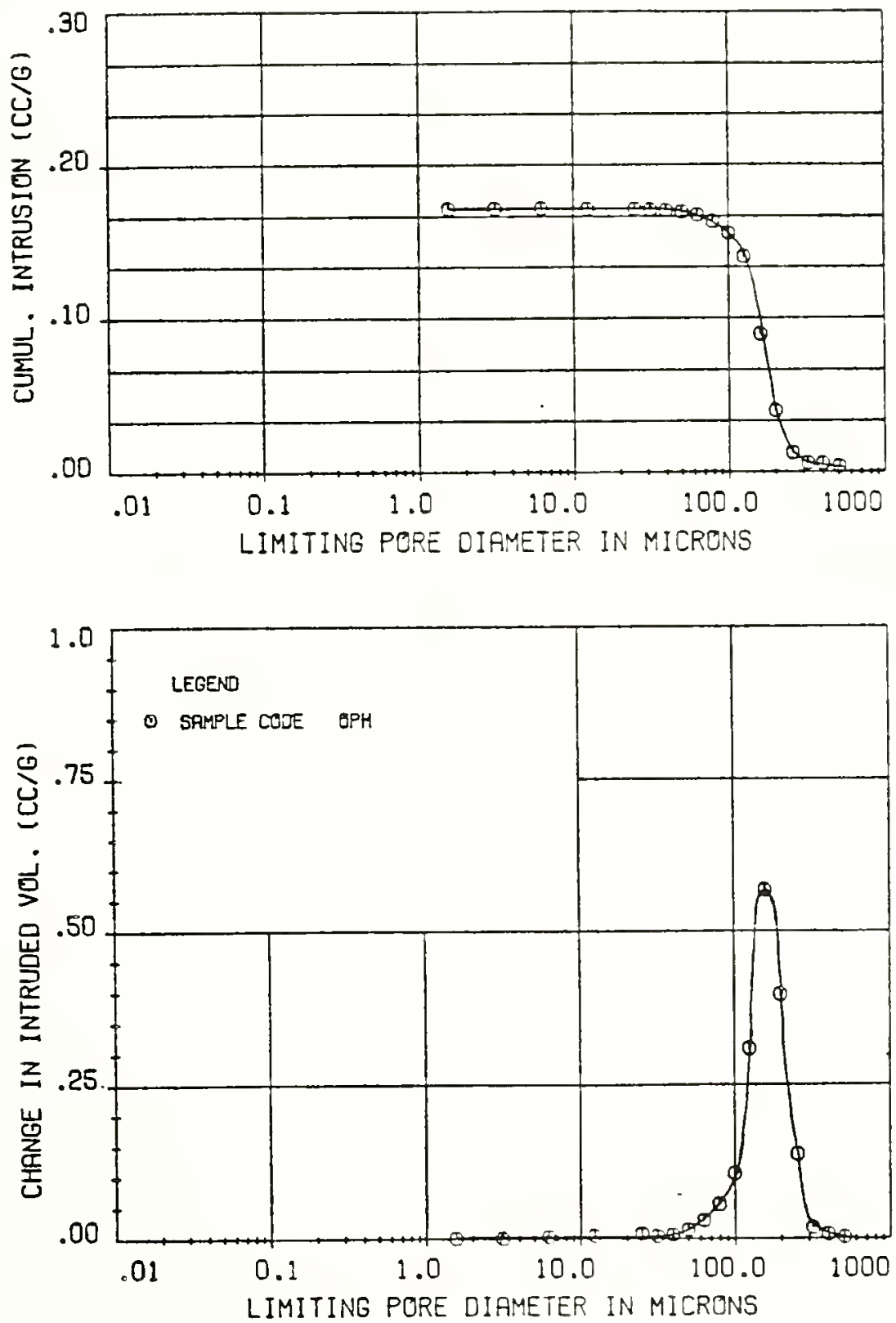

Figure 4-15 PSD Curves of OPH 
descriptors for the soil fabric.

For the purpose of correlating the theoretical permeability model with the pore size distribution data, it is preferable to express the cumulative distribution and density function of pore size as a percentage. It should be noted that at the maximum pressure exerted, about 10 to 15 percent of the pore space was not intruded for the solls tested. The reasons for this have not been established with certainty. Some of the pores may have entry ways too narrow to penetrate with mercury at $103,500 \mathrm{kPa}(15,000 \mathrm{psi})$, and most likely some of them may be in a network of pores not continuous with the boundaries of the specimen. Moreover, a minor shrinkage of the specimens during sample drying could account for part of the discrepancy.

A significant unintruded pore space is not unusual for pore size distributions determined by the mercury intrusion technique. About $25 \%$ of the pore space was not intruded by mercury in the study by Diamond, et al. (1972) for hydrated cement paste. A value of $20 \%$ unintruded pore space was reported by Lohnes, et al. (1976) for selected Hawaiian soils (e.g., Manana soil, consisting of $70 \%$ sand, $16 \%$ silt and $14 \%$ clay). A $45 \%$ unintruded pore space was reported by White (1980) for a medium plastic clay, St. Croix clay. However, only about $5 \%$ unintruded pore space was reported by Garcia-Bengochea (1978) and Reed.(1977) for clay-silt mixtures. Note that the above values are all under the maximum intruded pressure of $103,500 \mathrm{kPa}(15,000 \mathrm{psi})$ except the one used by Diamond, et al. (1972), in which a maximun pressure of $517,500 \mathrm{kPa}(50,000 \mathrm{ps} 1)$ had been applied. With these facts in mind, the discontinuity of pores may be the main reason of the existence of the unintruded pore space 
if a proper specimen drying method is used. However, Reed, et al.

(1980) have expressed the view that nearly all pore space in soil systems is connected.

Fortunately, this unintruded pore space effect is oi minor importance in this study which deals with flow through porous materials, because the pore channels through which water passes are probably larger than those penetrated by mercury at pressure of 15,000 psi.

An attempt at a correction for this unintruded pore space eifect was made by Meyer (1953). A modified form of his work is given in Appendix $\mathrm{A}-7$

Because of this unintruded pore space, the graphical results of the pore size distribution and its density function are presented in both manners discussed in Section 4.3.2. An example is shown in Figures 4-16 and 4-17. The rest of them are shown in Appendix A-8.

\subsubsection{Comparison of Pore Size Curves}

Figures $4-18,4-19$, and 4-20 show the effect of water content on pore size distribution. For SC3M samples (see Figure 4-18), the pore size density function shows a bimodal characteristic with differences in the large pore mode, and almost no changes in the small pore mode. The large pore mode decreases as water content increases from dry of optinum to optimum water content. There is little difference in the large pore mode of the optimum and the wet side samples. In general, the trend as indicated above is similar to that found by GarciaBengochea (1978).

Comparing the small pore modes displayed in Figures 4-18 and 4-19, i.t is evident that the small pore mode is almost the same in spite of 

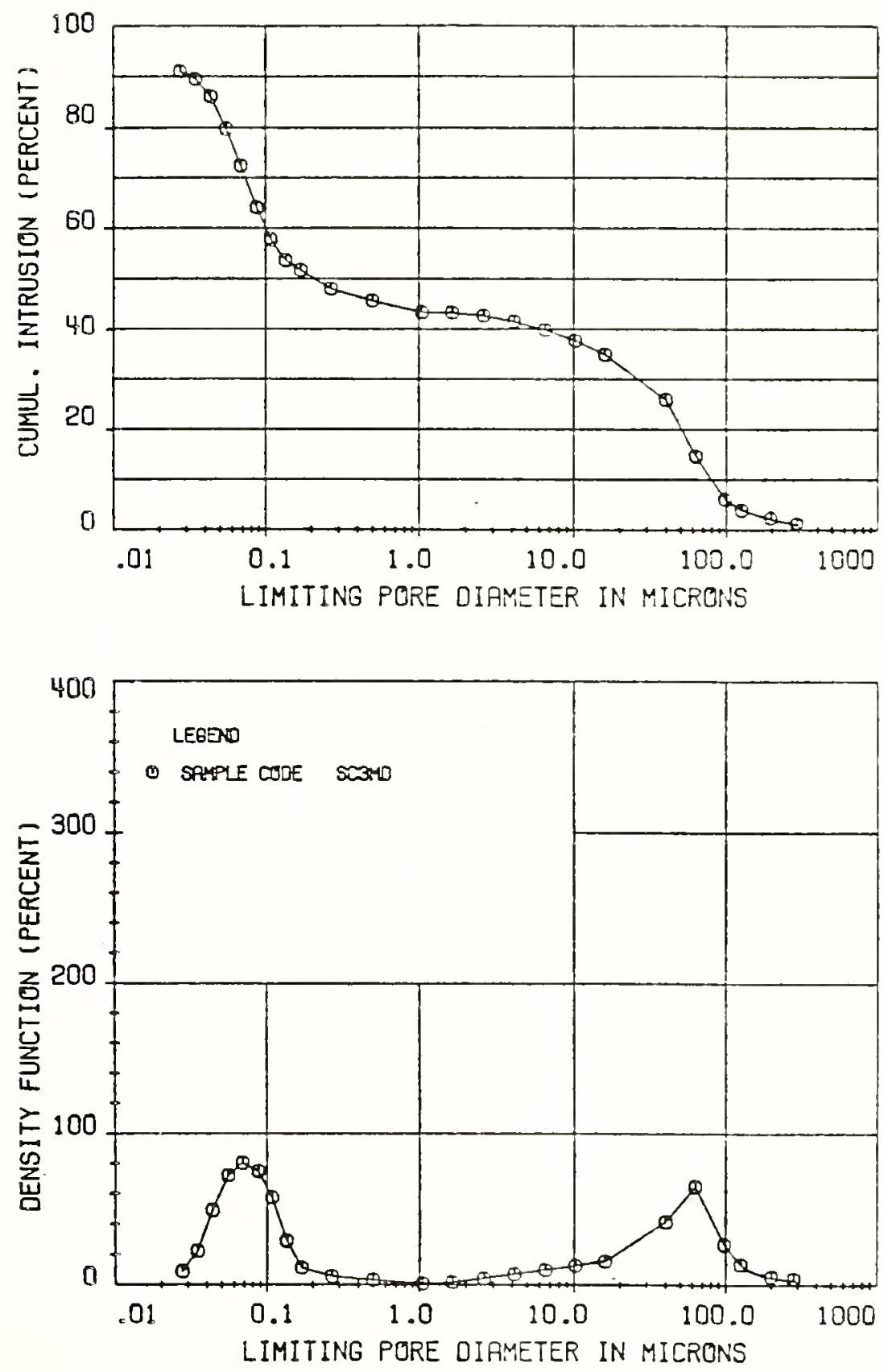

Figure 4-16 PSD Curves of SC3MD (Percent of Total Pore Volume Existing) 

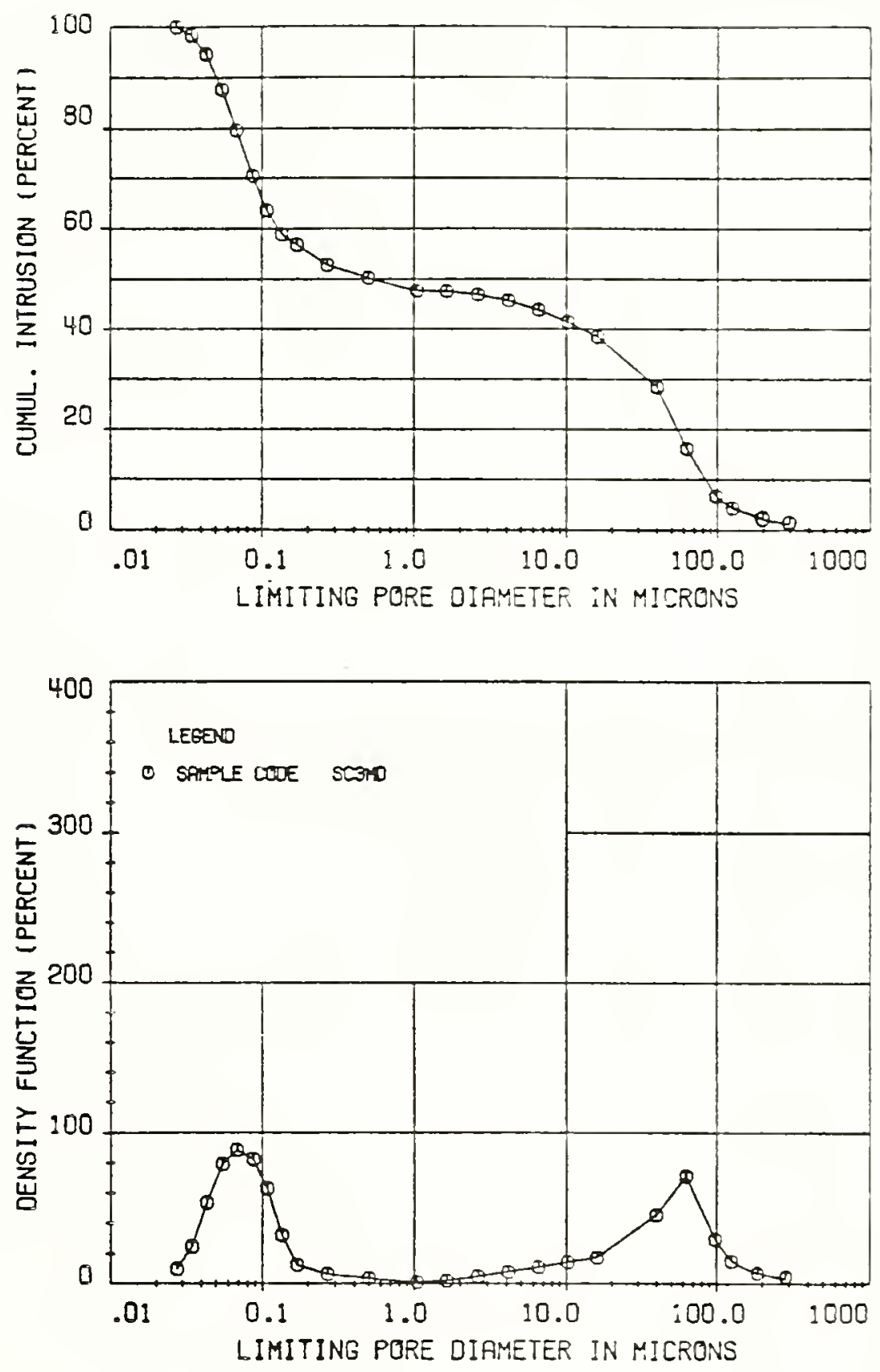

Figure 4-17 PSD Curves of SC3MD (Percent of Total Pore Volume Intruded) 

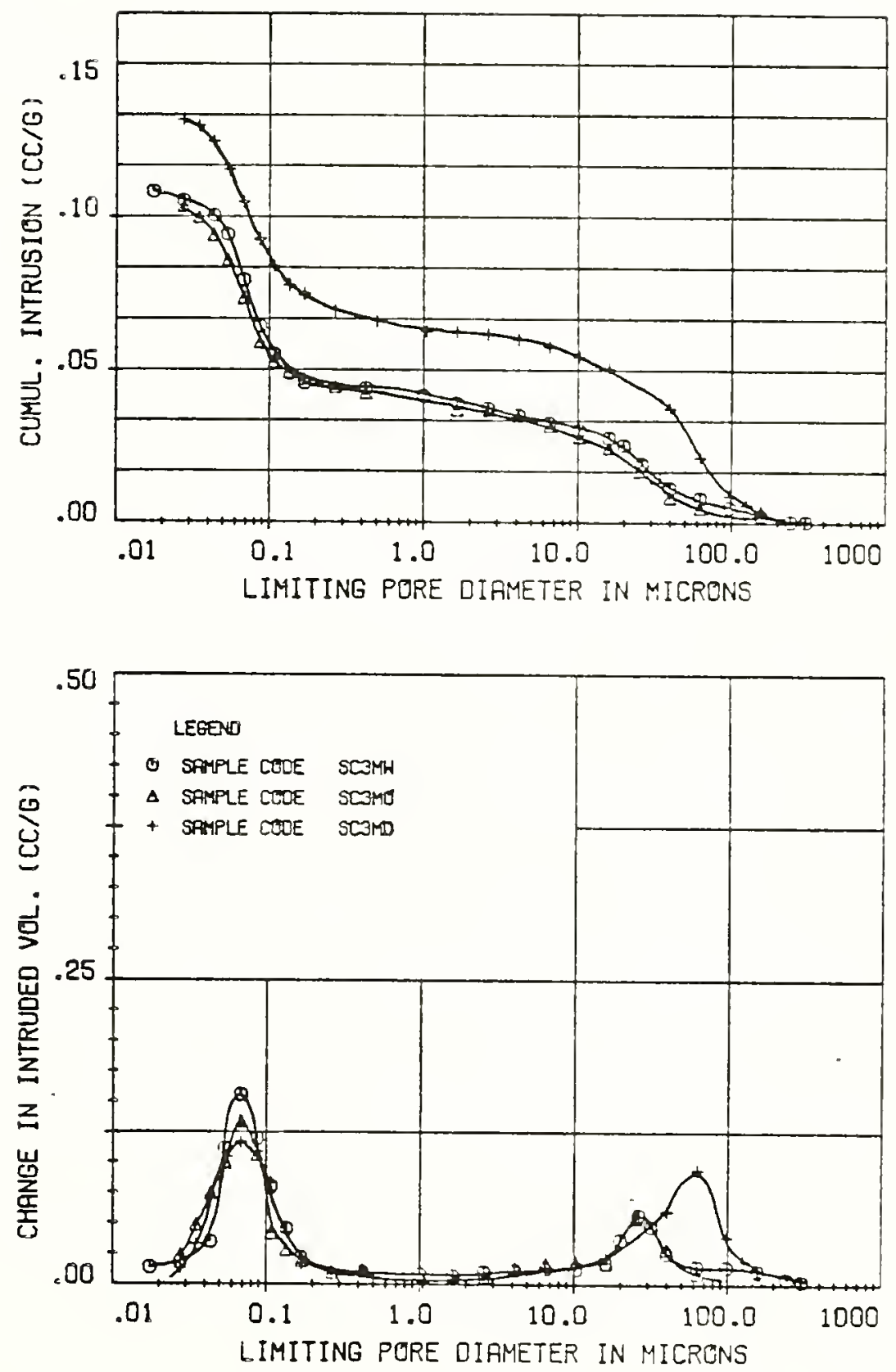

Figure 4-18 Comparison of PSD Curves for Samples Compacted at Different Water Contents ( SC $3 M$ series) 

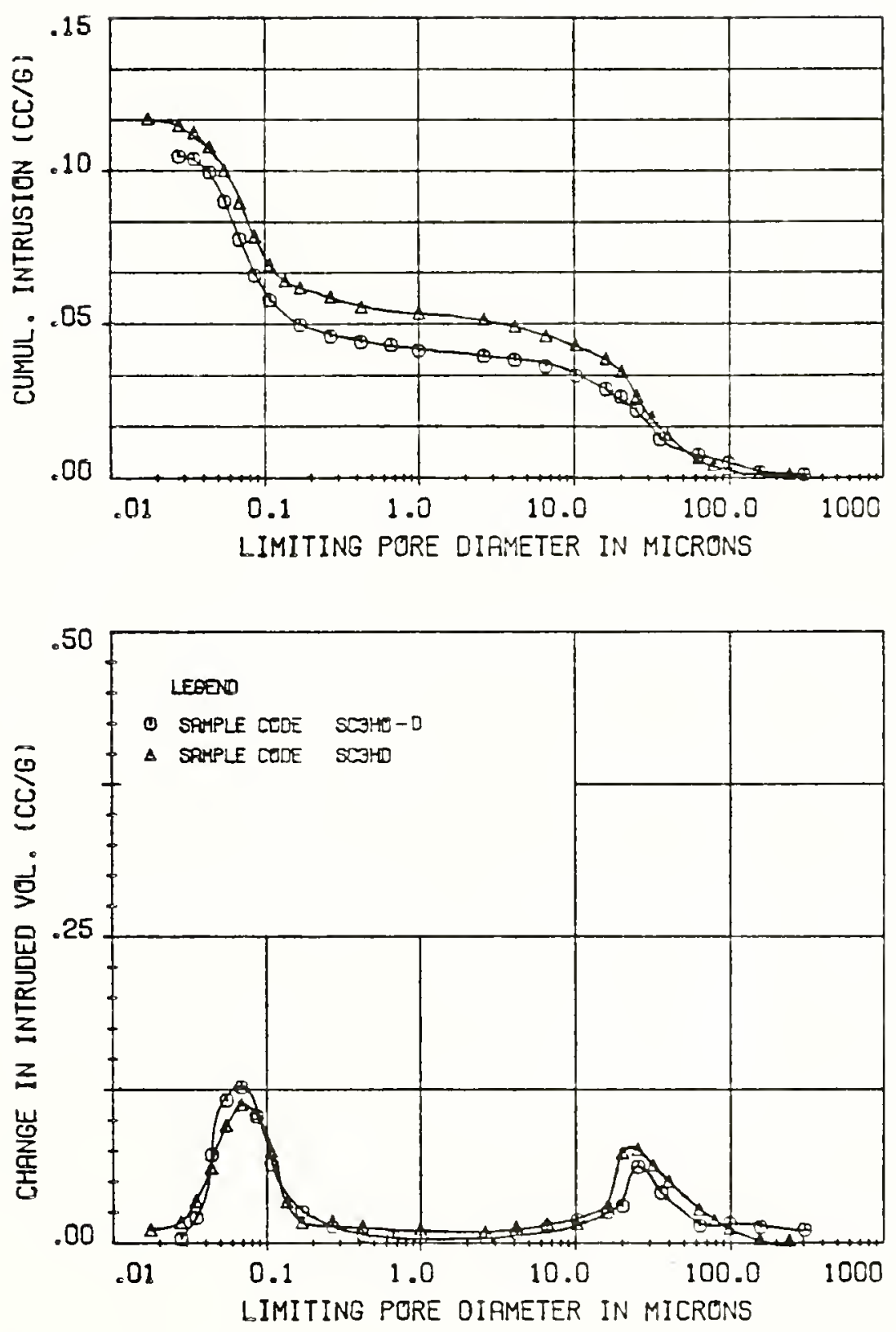

Figure 4-19 Comparison of PSD Curves for Samples Compacted at Different Water Contents (SC3H series) 

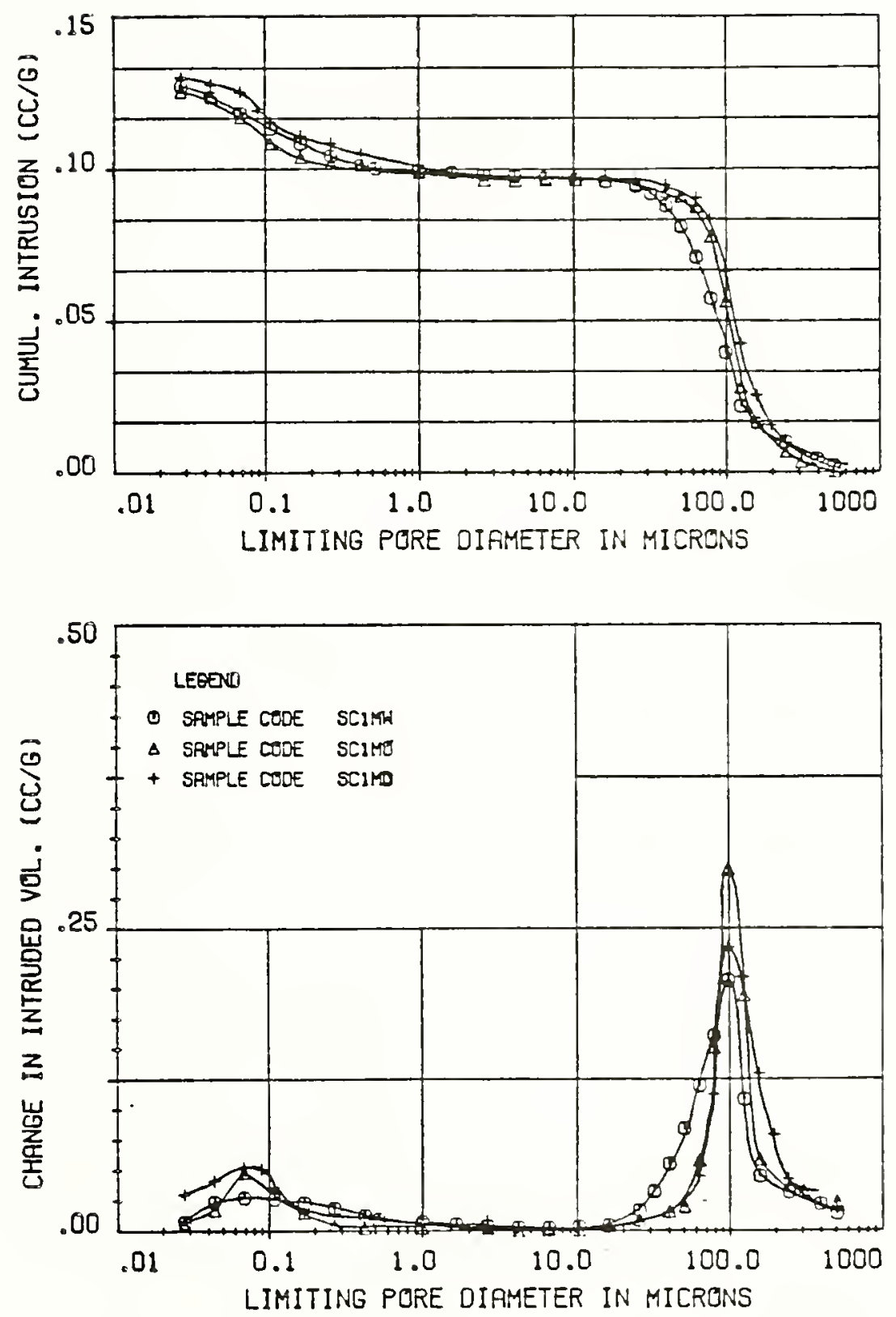

Figure 4-20 Comparison of PSD Curves for Samples Compacted at Different Water Contents (SCIM series) 
an increase of the compactive effort. This phenomenon will be discussed later. Unlike the trend in Figure 4-18, the large pore modes of the dry side and the optimum samples are quite similar in Figure 4-19, although they do show a more significant difference in the cumulative distribution curves. This may be due to inaccurate location of points of the optimum samples (see Figure 4-1). The optimum samples were indeed slightly dry of optimum water content, thus the samples were coded as SC3HO-D. Comparing results of permeability tf.sts (see Table 4-6), the difference between permeability of Sample SC3HD and Sample SC3HO-D is much smaller than that of Sample SC3MD and Sample SC3MO. The similar trend when comparing pore size characteristics and those of permeability implies that pore size distribution could be a sensitive indicator of permeability.

Figure 4-20 shows that the difference between pori size distribution of the dry side and optimum or wet side samples is small. The reasons noted in Section 4-2 for comparison of permeability measurements on the same soil compacted at different water contents may also contribute to the small differences between these pore size characteristics.

As noted in Section 2.1.3, seepage occurs only through the pores, and what is directly related to the permeability is the characteristics of the pores themselves. Thus the permeability of each of the samples increases with increasing large pore mode. Insignificant difference in the large pore mode is reflected by insignificint difference in permeability.

Figure 4-21 and Figure 4-22 show the effect of compactive effort on pore size characteristics. Note that these comparisons are only semi- 

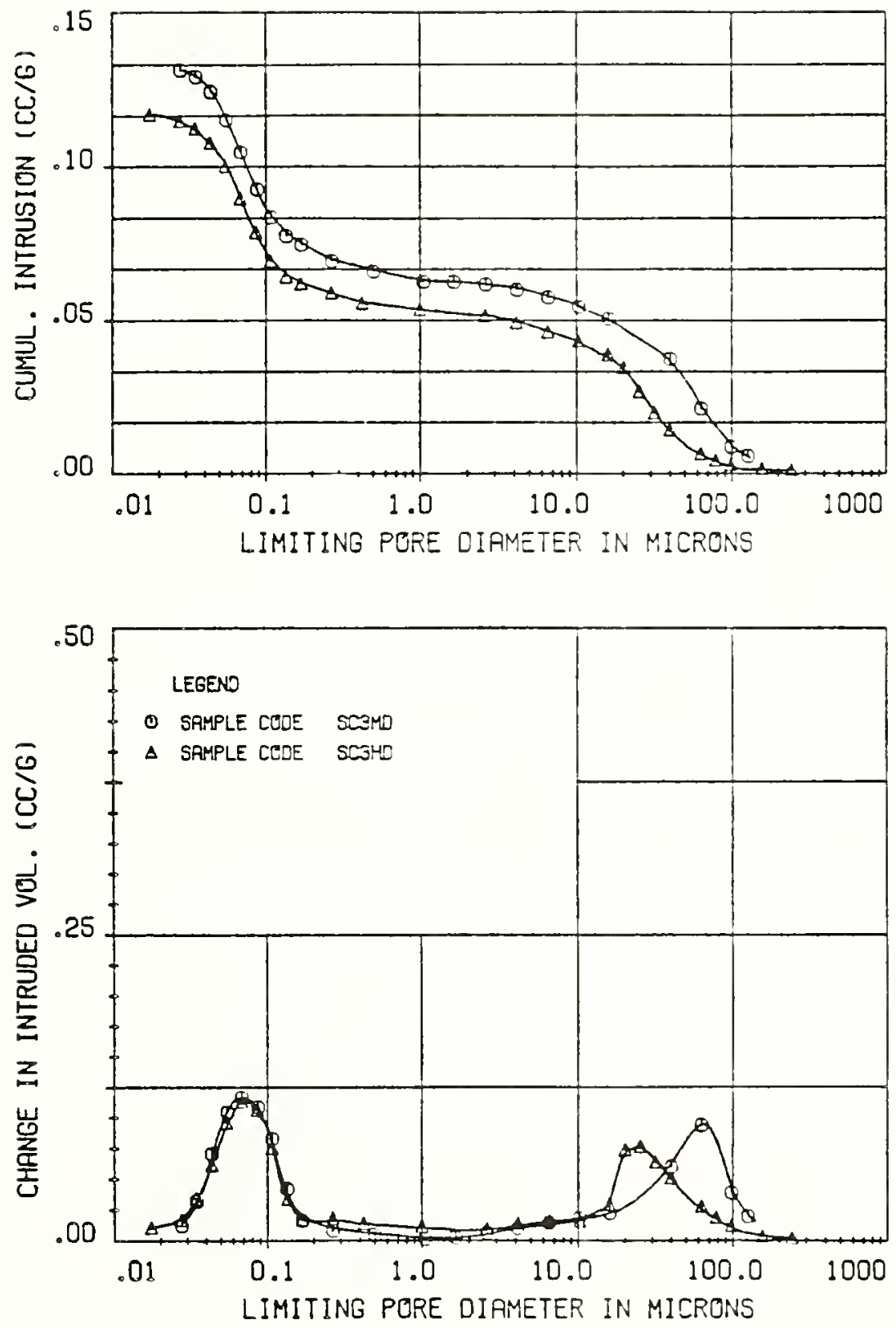

Figure 4-21 Comparison of PSD Curves for Samples Compacted at Different Compactive Efforts (Soil C) 

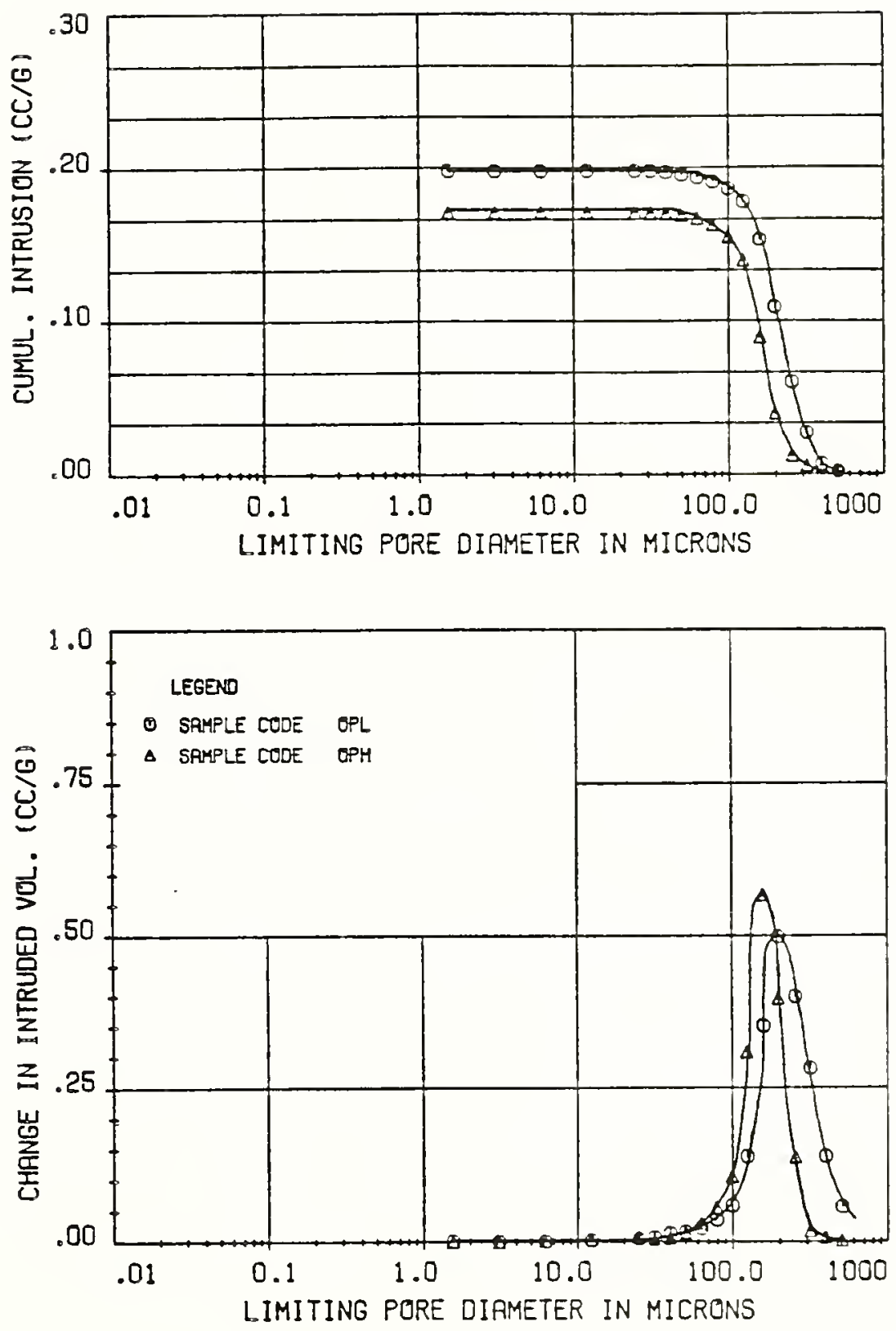

Figure 4-22 Comparison of PSD Curves for Samples Compacted at Different Compactive Efforts (Soil A) 
qualitative because the water content was not controlled at the same value. Figure 4-21 shows that as the compactive effort was increased, the large pore mode decreased, while the small pore mode remains unchanged. Figure 4-22 also shows the same trend except that there is no small pore mode. Recalling the permeability of these soils, again it decreases with decreasing large pore mode. This trend was also found by Garcia-Bengochea (1978).

Figures 4-23, 4-24 and 4-25 compare the pore size characteristics of Soils B and C compacted by medium effort dry of optimum, optimum and wet of optimum water contents, respectively. The large pore mode of the soils decreases with increasing clay content, while the small pore mode remains constant, but its probability density increases with increasing clay content.

Figure 4-26 compares the pore size characteristics of Soils A, $B$, and $C$ with different compaction variables. The small pore mode remains the same in spite of differences in compactive effort and amount of clay, while its probability density decreases with decreasing clay content. Note that there is no small pore mode in soil A, which follows from the fact that Soil A has no clay content. The large pore mode of the soils increases with increasing sand content as mentioned above. Comparing the permeability values and pore size characteristics of these soils (see Table 4-6 and Figure 4-26) shows that the permeability decreases with a decrease in the large pore mode. For the soils tested, the large pore mode and its probability density appears to be the best indicator of changes in soil fabric caused by varying water content, compactive effort, and soll type. This was also found by Garcia-Bengochea (1978). 

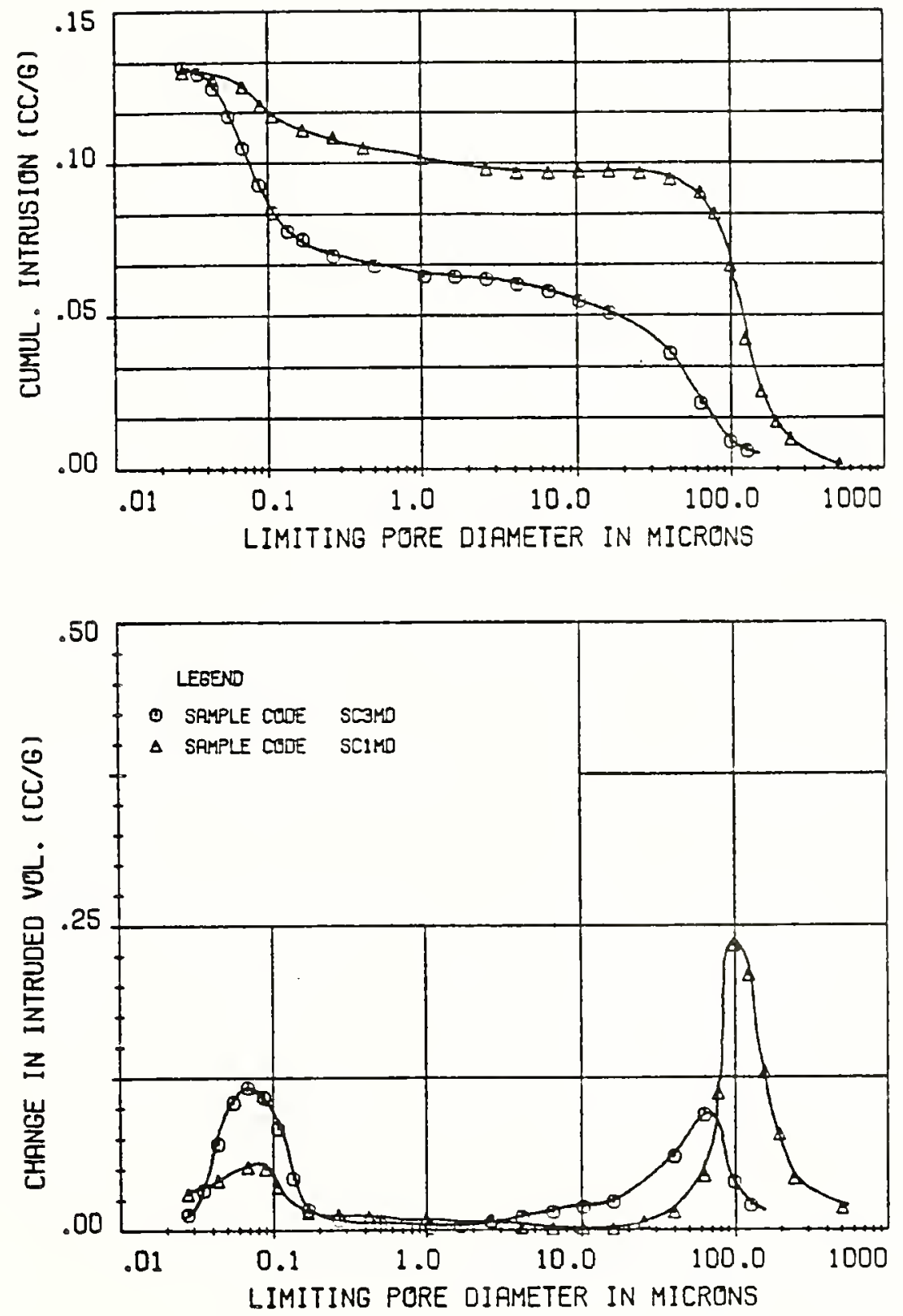

Figure 4-23 Comparison of PSD Curves for Different Soils ( $B$ and $C$ ) Compacted by Medium Effort at Dry of Optimum Water Content 

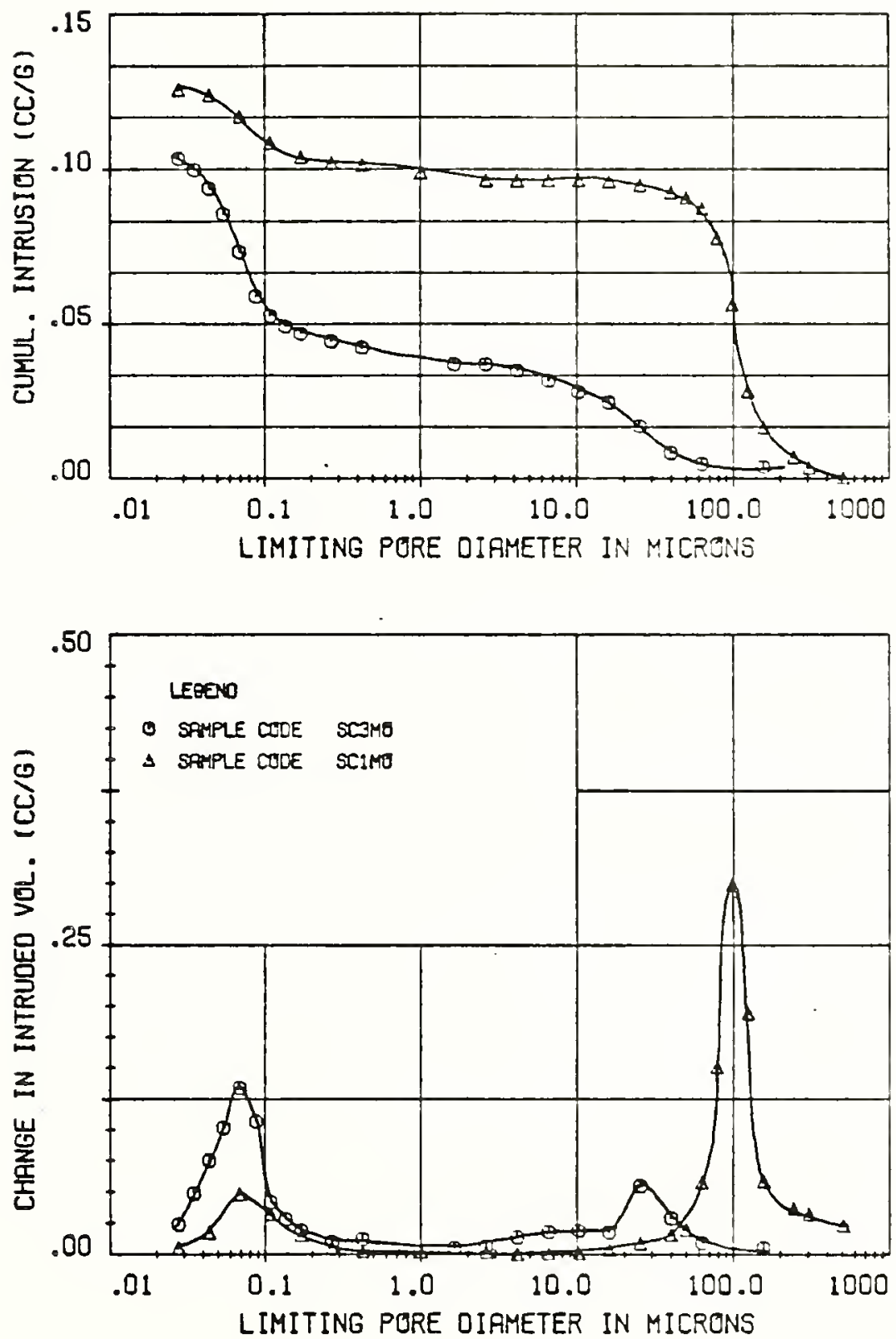

Figure 4-24 Comparison of PSD Curves for Different Soils ( $B$ and $C$ ) Compacted by Medium Effort at Optimum Water Content 

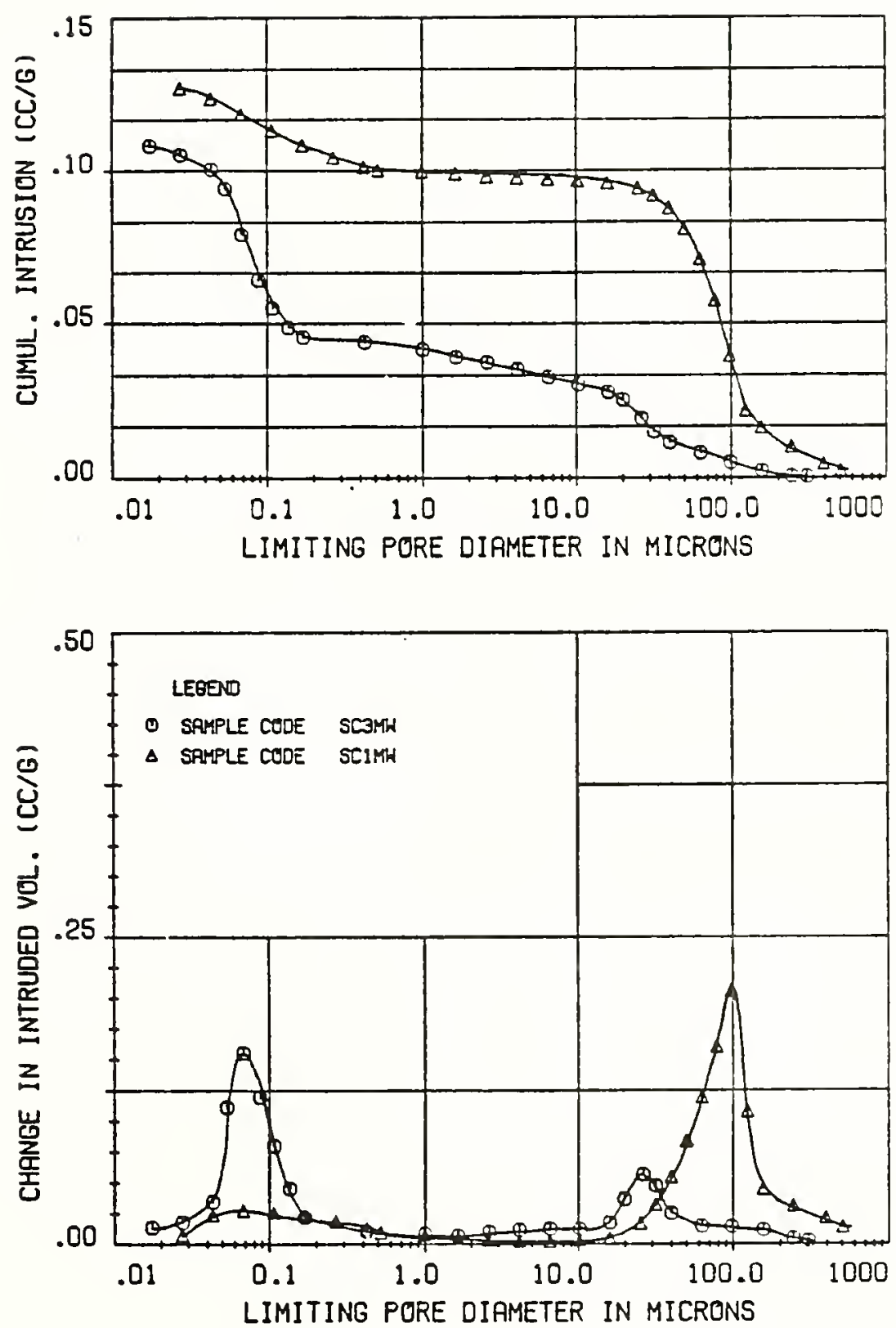

Figure 4-25 Comparison of PSD Curves for Different Soils ( $B$ and C) Compacted by Medium Effort at Wet of Optimum Water Content 

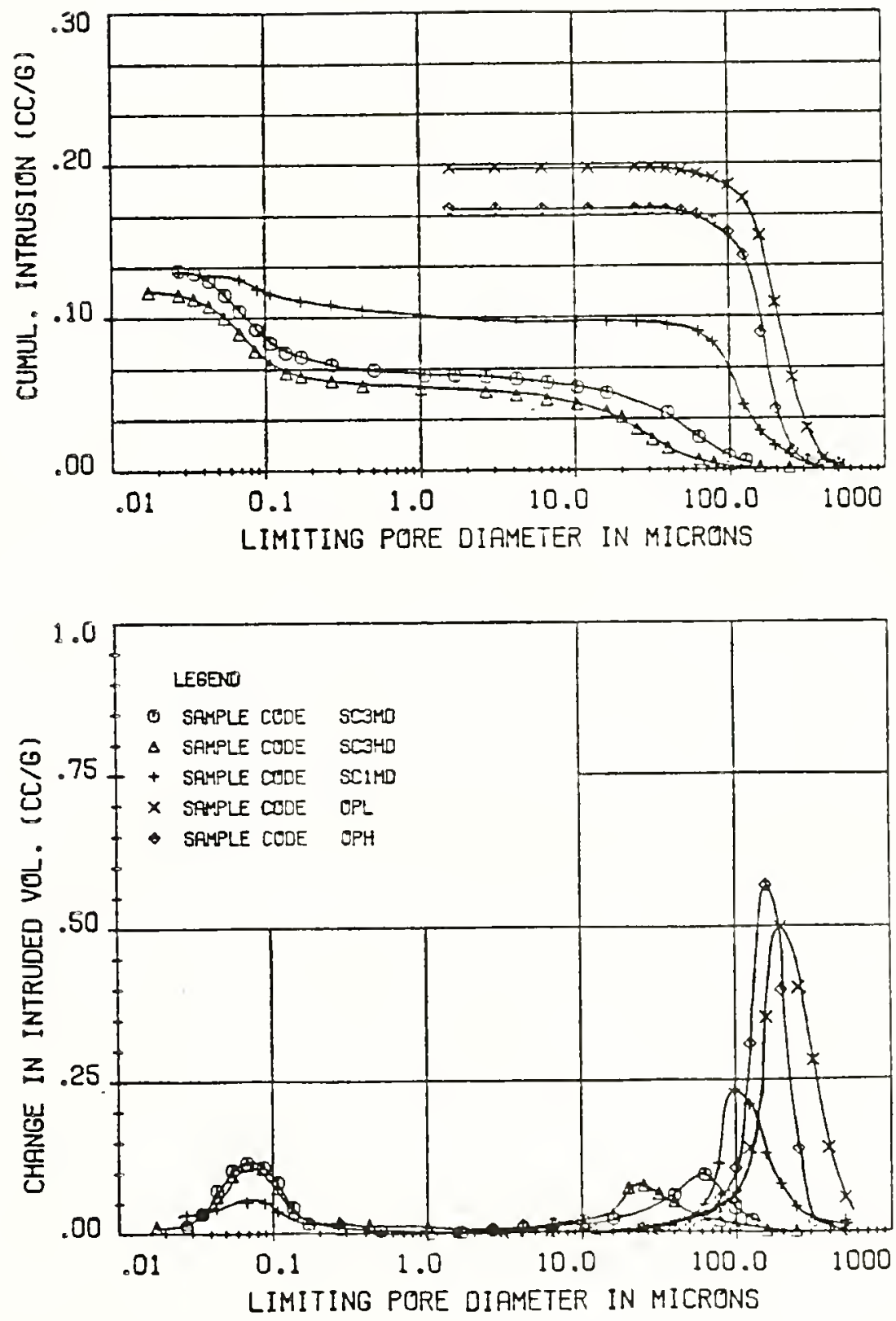

Figure 4-26 Comparison of PSD Curves for Different Soils ( $A, B$ and $C$ ) 
The hypothesis, proposed by Garcla-Bengochea (1978), that the shape of the differential pore size distribution (called pore size density function in present study) in the small pore region (referred to as small pore mode) may serve as a "signature" of a given clay type, is supported by the data in this study. Again, the small pore mode is related to the size range of clay particles present, and the probability density of the small pore mode is in proportion to the amount of clay minerals in the soil.

\subsection{PREDICTION OF PERMEABILITY BY PROPOSED MODEL}

The objective of this section is to correlate the permeability and the PSD of the selected soils by applying the theoretical model proposed in Section 2.2.2.

Before going into a detailed discussion of the proposed model, it is worthwhile to examine the following functional relationships between void ratio, e and permeability, $k$ as presented by Taylor (1948) and Lambe (1951):
(a) $k$ vs $\cdot e^{3} /(1+e)$
(b) k vs. $e^{2} /(1+e)$
(c) k vs. $e^{2}$
(d) $\log \mathrm{k}$ vs. e

Since the permeabilities of the soils studied vary over five orders of magnitude, it is very unlikely that linear relationships for these relationships exist. Therefore the logarithm of $k$, instead of $k$, was used for the regression analyses. The statistical programs of the Statistical Package for the Social Sciences (SPSS) in the Purdue University CDC 6500-6600 computer library were used to do least squares analysis and regressions in this study. The coefficient of determination 
$\left(R^{2}\right)$ of the regressions of $k$ with respect to the functional parameters of $\mathrm{e}$ mentioned above are shown in Table 4-7. Although $\mathrm{R}^{2}$ was around 0.6 , the differences between the measured and the calculated permeabilities are generally greater than one order of magnitude and, in some samples are greater than two orders of magnitude. The results noted above imply that gross volumetric parameters (e.g. void ratio or porosity and their functions) cannot appropriately account for changes in permeability of compacted soils.

Previous discussions in Sertion 2.2 provided the theoretical background for predicting the permeability from the PSD of soils. From the qualitative observattons discussed in Section 4.3 .4 , it is evident that the permeability is related to the distribution of pore sizes about the large pore mode. Moreover, the proposed model is very sensitive to the larger pore diameters (see Equation 2-19). Therefore the pores of diameter less than $1 \mu m$ were considered to be negligible in the calculation of permeability. A physical explanation of choosing lum as the lower limit of pore sizes which is accessible to flow is the concept of "threshold diameter" given by Murota and Sato (1969). In their study, a threshold pore diameter of $1.8 \mu \mathrm{m}$ was chosen for silty clay. On the other hand, the upper $11 \mathrm{mit}$ of pore sizes in this study was bounded by the pressure capacity of the mercury porosimetry. Hence Equation (2-19) can be rewritten as:

$$
k=\frac{y n^{2}}{32 \mu} \int_{1}^{x_{\max }} \int_{1}^{x_{\max }} \tilde{x}^{2} G\left(x_{j}\right) \cdot f\left(x_{i}\right) \cdot f\left(x_{j}\right) d x_{i} d x_{j}
$$

Numerical integration of Equation (4-12) was accomplished by a computer program 11sted in Appendix A-9. Note that the equivalent diameter, $\tilde{x}$ was assumed to be the smaller of each pair of connected 


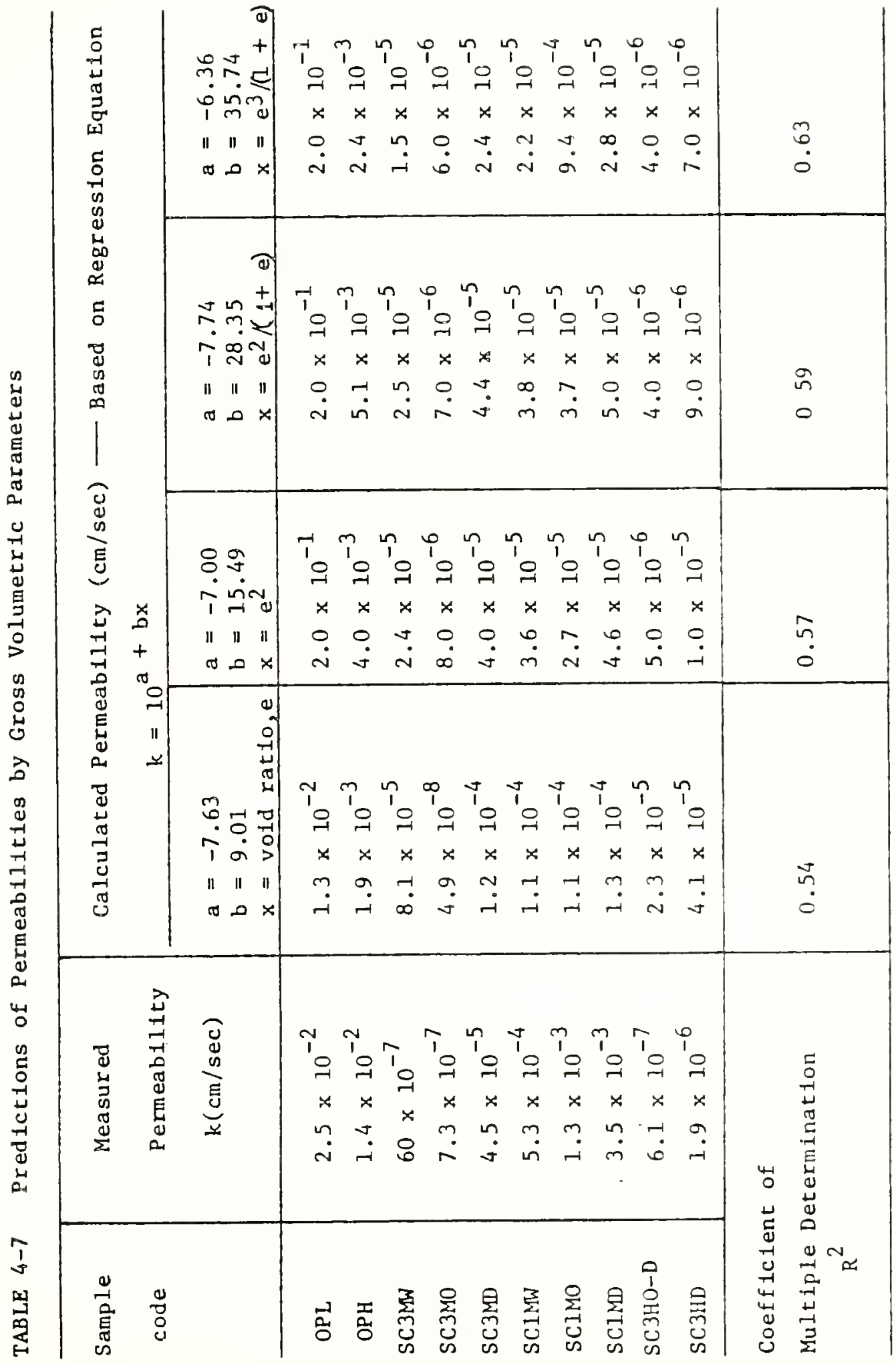


pores. The term $G\left(x_{j}\right)$ defined in Equation (2-18) contains a undetermined function, $V$. Thus in order to predict permeability from Equation (4-12), $\mathrm{V}$ must be assumed. In this study, $\mathrm{V}$ was assumed to be a linear function of pore diameter (i.e. $v\left(x_{j}\right)=v \cdot x_{j}$ ) in order to simplify the calculations. The pore size density function $f$ was determined previously (see Section 4.3.3 and Appendix A-5) and was expressed as a dimensionless term. In other words, $f$ was a piecewise cubic function which fitted its corresponding PSD curve (i.e., Figures A-8-1 through $A-8-20)$.

The computer program listed in Appendix A-9 provides an iterative method to seek a suitable value of $v$ at whuch a good prediction of permeability is obtained. In this computer program the criterion of a good prediction of permeability is determined by the user. In the present study, a criterion of a $15 \%$ error with respect to the measured permeability was arbitarily chosen. Two other criteria were number of iterations and the relative change of the calculated permeability between the two successive values of $v$. For each iterative run, the main feature of the computer program listed is the numerical integration of the double integral in Equation (4-12). A subroutine called "CADRE" in the Purdue University CDC 6500-6600 computer library was utilized to carry out this numerical integration.

The output of the interations discussed above for the samples tested is also given in Appendix A-9.

Following the above discussions and the results given in Appendix A-9, the values of $v$ for all samples studied are listed in Table 4-8. The next step is intended to correlate $v$ to the pore size characteristics of soils. Table 4-9 lists some of the possible 
TABLE 4-8 The $v$ - Values

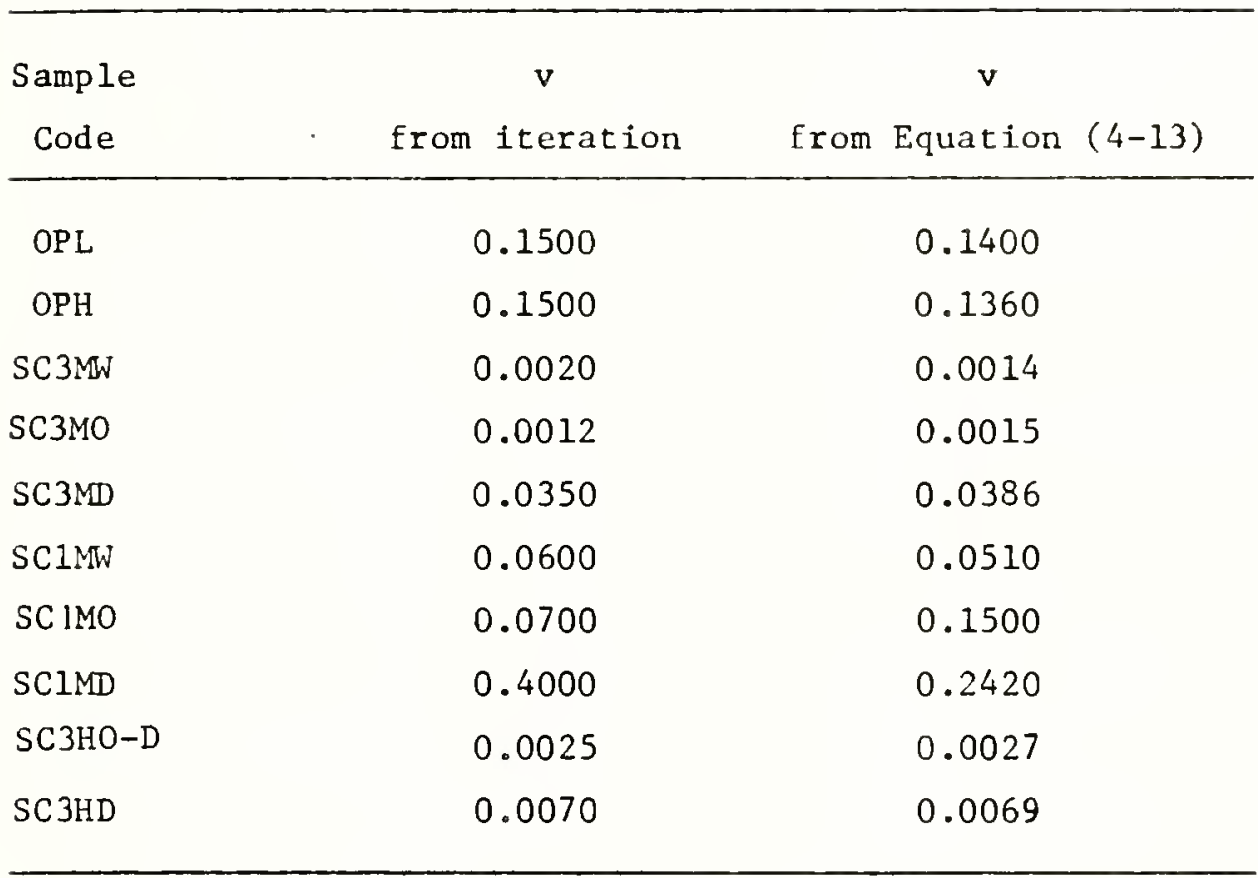


TABLE 4-9 Possible Correlations between PSD

Parameters and $v$

Dependent Variable

Independent Variable

(PSD parameters)

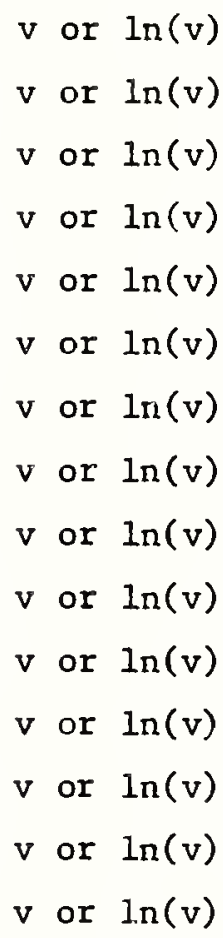

$\mathrm{D}_{25}$ (see remarks)

$\mathrm{D}_{50}$

$\mathrm{D}_{75}$

$\mathrm{LD}_{25}$ (see remarks)

$\mathrm{LD}_{50}$

$\mathrm{LD}_{75}$

$\mathrm{D}_{25}, \mathrm{D}_{50}, \mathrm{D}_{75}$

$\mathrm{LD}_{25}, \mathrm{LD}_{50}, \mathrm{LD}_{75}$

$\mathrm{D}_{40} / \mathrm{D}_{80}$

$\mathrm{D}_{30}^{2} /\left(\mathrm{D}_{10} \times \mathrm{D}_{60}\right)$

$\mathrm{D}_{60} / \mathrm{D}_{10}$

$\mathrm{D}_{40} / \mathrm{D}_{25}$

$\mathrm{D}_{40} / \mathrm{D}_{10}$

$\mathrm{D}_{60} / \mathrm{D}_{10}, \mathrm{D}_{40} / \mathrm{D}_{25}, \mathrm{D}_{40} / \mathrm{D}_{10}$

$\mathrm{D}_{60} / \mathrm{D}_{25}, \mathrm{D}_{60} / \mathrm{D}_{40}, \mathrm{D}_{25} / \mathrm{D}_{10}$

Remarks :

a) $\mathrm{D}_{25}$ means 25 Percentile (a diameter) of the cumulative distribution curve, i.e., the subscripts represent the percentile of the distribution.

b) $\mathrm{LD}_{25}$ means the $\log$ of diameter $\mathrm{D}_{25}$ 
correlations between PSD parameters and v-values. Simple linear regression by the method of least squares was used to evaluate these possible correlations, and the following satisfactory linear relation was established with $R^{2}=0.97$ and an adjusted $R_{a}^{2}=0.96$ :

$$
\ln v=-6.15-40.07\left(\frac{\mathrm{D}_{40}}{\mathrm{D}_{25}}\right)+118.79\left(\frac{\mathrm{D}_{40}}{\mathrm{D}_{10}}\right)-78.35\left(\frac{\mathrm{D}_{60}}{\mathrm{D}_{10}}\right)
$$

With Equation (4-13) and the PSD of soils, the value of $v$ can be determined (see Table 4-8). The permeability can then be calculated by using the computer program described above. Table 4-10 compares the calculated and the measured permeabilities. A linear regression was performed and the result as shown in Figure 4-27 was excellent.

In summary, the proposed permeability model can provide an excellent predictive tool. Thus a functional relationship between the PSD and the permeability of compacted soils is clearly demonstrated.

\subsection{APPLICABILITY OF THE PROPOSED PERMEABILITY MODEL}

Attempts were made to broaden the range of prediction of zermeability using independently determined data. Ten sets of PSD curves along with permeability values were taken from GarciaBengochea (1978). The procedures described above were carried out for both the author's and Garcia-Bengochea's data. The following regression equation was established for the v-value $\left(R^{2}=0.89\right)$ :

$$
\begin{aligned}
\ln v= & -3.6031-2.5957\left(\frac{\mathrm{D}_{40}}{\mathrm{D}_{25}}\right)+14.9678\left(\frac{\mathrm{D}_{40}}{\mathrm{D}_{10}}\right) \\
& -7.2562\left(\frac{\mathrm{D}_{60}}{\mathrm{D}_{10}}\right)+4.6320\left(\frac{\mathrm{D}_{60}}{\mathrm{D}_{25}}\right)-2.7129\left(\frac{\mathrm{D}_{25}}{\mathrm{D}_{10}}\right) \\
& -3.8473\left(\frac{\mathrm{D}_{60}}{\mathrm{D}_{40}}\right)
\end{aligned}
$$


TABLE 4-10 Comparison of Predicted and Measured Permeability

\begin{tabular}{lcc}
\hline $\begin{array}{l}\text { Sample Code } \\
(\mathrm{cm} / \mathrm{sec})\end{array}$ & $\begin{array}{c}\text { Predicted Permeability } \\
\text { OPL }\end{array}$ & $\begin{array}{c}\text { Measured Permeability } \\
(\mathrm{cm} / \mathrm{sec})\end{array}$ \\
\hline OPH & $1.43 \times 10^{-2}$ & $2.5 \times 10^{-2}$ \\
SC3MW & $4.40 \times 10^{-7}$ & $1.4 \times 10^{-2}$ \\
SC3MO & $9.85 \times 10^{-7}$ & $6.0 \times 10^{-7}$ \\
SC3MD & $4.85 \times 10^{-5}$ & $7.3 \times 10^{-7}$ \\
SC1MW & $4.98 \times 10^{-4}$ & $4.5 \times 10^{-5}$ \\
SC1MO & $2.24 \times 10^{-3}$ & $5.3 \times 10^{-4}$ \\
SC1MD & $2.40 \times 10^{-3}$ & $1.3 \times 10^{-3}$ \\
SC3HO-D & $7.31 \times 10^{-7}$ & $3.5 \times 10^{-3}$ \\
SC3HD & $1.70 \times 10^{-6}$ & $6.1 \times 10^{-7}$ \\
\hline
\end{tabular}




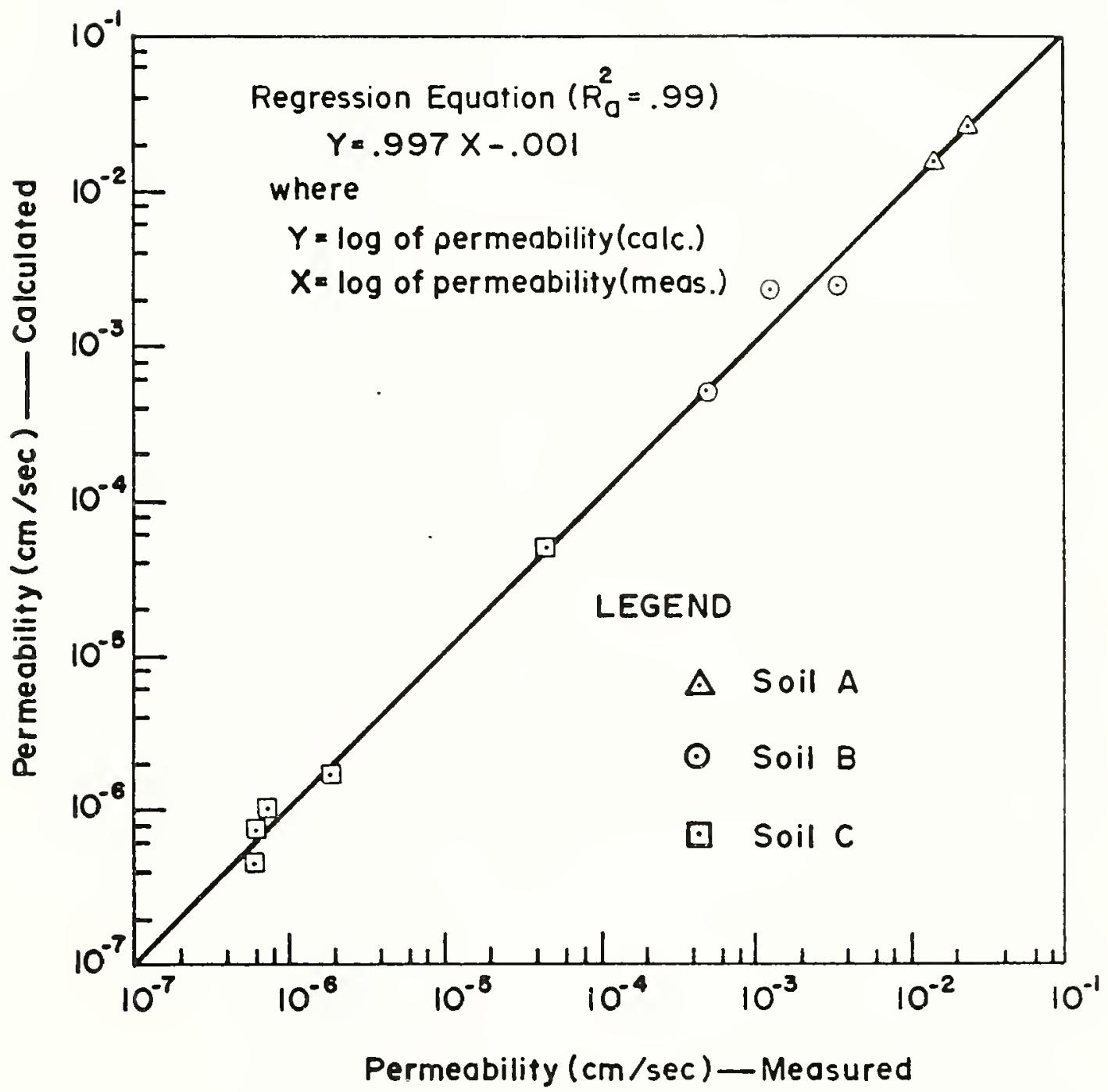

Figure 4-27 Prediction of Permeability by Proposed Model 
The values of $v$ calculated from Equation (4--14) were then used In the calculation of permeability. The information from each data set, including sample code, PSD curve-fitting result, the v-value, and the calculated and measured permeabilities are given in Appendix A-10. Note that the computer program listed in Appendix A-9 was used to perform the above calculations. In addition, a regression analysis was performed on the permeability data (i.e., the calculated and the measured permeability). The result is shown in Figure 4-28. It is evident that the proposed permeability model provides as excellent theoretical tool for the prediction of permeability for a wide range of soil types. 


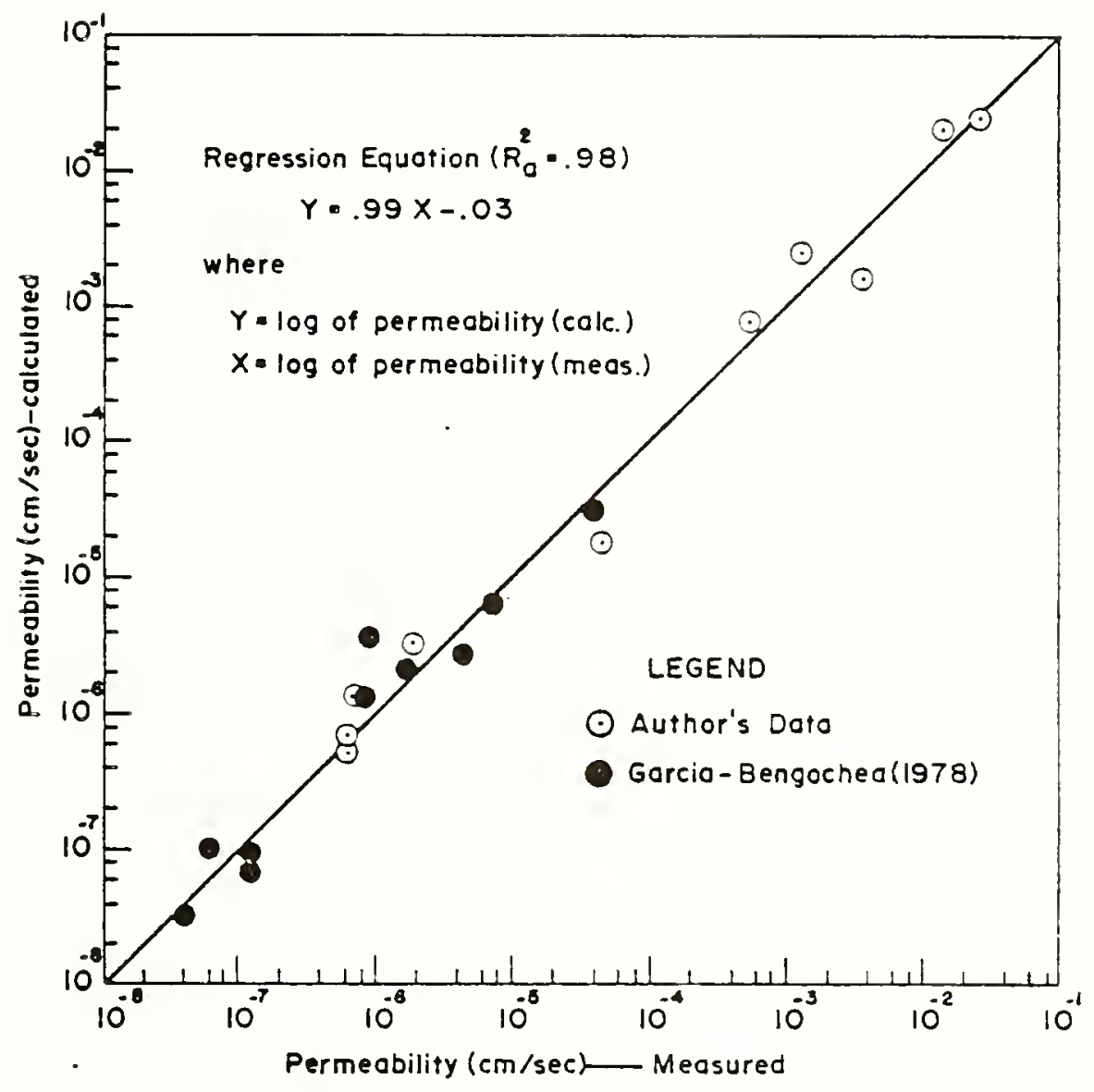

Figure 4-28 Prediction of Permeability by Proposed Model (Author's and Garcia-Bengochea's Data) 
CHAPTER V SUMMARY, CONCLUSIONS AND RECOMRENDATIONS

\subsection{SUMMARY}

The theoretical background for the determination of the pore size distribution of soils has been critically reviewed. Mathematical interpretations of PSD were given in detail. For the soils studied, both the pore size distributions and pore size density functions were presented. The pore size distributions from original PSD data were expressed as intruded volume per gram of specimen. In addition, they were also expressed as a dimensionless term (e.g., percentage) Ẽor convenient mathematical treatment. The pore size density function could be obtained either from direct differentiation of a curve-fitted cumulative distribution function, or by finite difference approximation. Moreover, the results of both methods agree very well.

The soils used in the study were a sand and two sand-clay mixtures. Pluvial compaction was used to prepare compacted samples of sand while kneading compaction was used to prepare samples of sand-clay mixtures. The testing program consisted of the permeability test and the PSD test. The experimental procedures of these tests for sand-clay mixtures (called Soils B and C in this study) generally followed GarciaBengochea (1978). For the sand (called Soil A ), a small amount, $0.5 \%$ by weight, of powdered phenolic resin was mixed with sand before compaction. Oven drying at a temperature of $170^{\circ} \mathrm{C}$ for $1 \mathrm{~h}$ was carried out after compaction in order to "areate a "cohesion". This technique 
enabled PSD specimens to be prepared and PSD tests to be conducted. Thus, the characterization of sand fabrics by their PSD was possible. Qualitative descriptions of the pore size characteristics of the solls studied were given through their pore size density functions. When displayed on a log diameter scale, the pore size density function of Soil A showed a single modal characteristic while those of soils $B$ and $C$ showed bimodal characteristics. The location of the mode (at which a peak was observed) and its probability density could serve as a useful qualitative descriptor. For the bimodal characteristics of Soils $B$ and $C$, the small pore mode occurred consistently around 0.1 $\mu \mathrm{m}$ and the large pore mode was between 10 and $100 \mu \mathrm{m}$. For the single modal characteristic of Soil A, the large pore mode occurred around $200 \mu \mathrm{m}$.

The hypothesis, proposed by Garcia-Bengochea (1978), that the shape of the differential pore size distribution (called pore size density function in the present study) in small pore region (referred to as small pore mode) may serve as a "signature" of a given clay type, is supported by the data obtained in this study. In addition, changes in soil fabric caused by varying water content and compactive effort were generally reflected by changes in large pore mode and its probability density.

The relation between the compaction variables and the permeability found in this study generally agree with the trends found by Lambe (1954), Mitchell, et al. (1965) and Garcia-Bengochea (1978). For the prediction of permeability, the relation between permeability and pore geometry was critically reviewed. A new permeability model based on Child and Collis-George's (1950) model was proposed. The 
new model had the following characteristics: (1) a probabilistic basis, (2) no arbitrary matching factor required, and (3) consideration of a tortuosity factor. The prediction of permeability by the proposed model was excellent $\left(\mathrm{e} . \mathrm{g}\right.$., the $\mathrm{R}_{\mathrm{a}}^{2}$ of regression of predicted permeability with respect to measured permeability equaled 0.99). Data obtained independently by Garcia-Bengochea (1978) was also used to examine the proposed model. The excellent result $\left[\mathrm{e} . \mathrm{g} \cdot, \mathrm{R}_{\mathrm{a}}^{2}=0.98\right.$ for both author's and Garcia-Bengochea's (1978) data] confirmed the applicability of proposed model.

\subsection{CONCLUSIONS}

1. A technique for the preparation of PSD specimens of cohesionless sands has been developed.

2. Finite difference approximation method is an appropriate approach for determining pore size density functions. The graphical presentation of pore size density function obtained from a finite difference approximation agrees very well with that obtained from direct differentiation of the curve-fitted cumulative distribution function.

3. The curve fitting technique using the computer program "SPLINE" for the PSD data of soils studied was apparently satisfactory.

4. Both pore size distribution and pore size density function are useful for characterizing the pore size characteristics of the soils studied in this research. The mode and its probability density of pore size density function curves served as successful qualitative descriptors, especially in characterizing the changes in soil fabric caused by varying compact- 
ion variables. The pore size parameters $(\mathrm{e} \cdot \mathrm{g} \cdot$, some percentile and their functions) obtained from pore size distributions were successfully used to predict the constant $v$ of the proposed permeability model.

5. The pore size density function of Soll A (Ottawa sand) was found to be singly modal on a log diameter scale. The mode occurred around $200 \mu \mathrm{m}$.

6. The pore size density functions of Soils B and C (sand-clay mixtures) were found to be bimodal on a $10 g$ diameter scale. A large pore mode occurred between $25 \mu \mathrm{m}$ and $100 \mu \mathrm{m}$, and a small pore mode occurred around $0.1 \mu \mathrm{m}$.

The following conclusions ( 7 through 12) verify conclusions previously reached by Garcia-Bengochea (1978).

7. The large pore mode decreased as water content increased from dry of optimum to optimum water content.

8. The large pore mode decreased as the compactive effort increased.

9. The large pore mode of the soils tested decreased with increasing clay content.

10. The small pore mode remained the same in spite of differences in compactive effort and amount of clay, while its probability density decreased with decreasing clay content.

11. For the soils tested, the large pore mode and its probability density appear to be the best indicators of changes in soil fabric caused by varying water content, compactive effort, and soil type. 
12. The hypothesis, proposed by Garcia-Bengochea (1978), that the shape of the differential pore size distribution (called pore size density function in the present study) in the small pore region (referred to as small pore mode) may serve as a "signature" of a given clay type, is supported by this study. The following conclusions ( 13 through 18) apply to the proposed permeability model.

13. The coefficient of determination $\left(R^{2}\right)$ of the conventional relation between permeability $k$ and void ratio e (e vs log k) was 0.54 for the soil samples tested. This result showed that $k$ can not be accurately predicted from total porosity or functions thereof.

14. A model has been proposed which relates PSD to soil permeability which is as follows:

$$
\begin{aligned}
& k=\frac{y n^{2}}{32 \mu} \int_{0}^{\infty} \int_{0}^{\infty} \tilde{x}^{2} \cdot G\left(x_{j}\right) \cdot f\left(x_{i}\right) \cdot f\left(x_{j}\right) \cdot d x_{i} d x_{j} \\
& \text { where } G\left(x_{j}\right)=e^{-\left(\frac{x_{i}-x_{j}}{V}\right)^{2}} \\
& \text { and } \\
& V=v \cdot x_{j}
\end{aligned}
$$

15. The following regression equation for the constant $v$ was obtained for the samples tested in this study $\left(R^{2}=0.97\right.$ and

$$
\begin{aligned}
\left.\mathrm{R}_{\mathrm{a}}^{2}=0.96\right): & \\
\ln \mathrm{v}=-6.15- & -40.07\left(\frac{\mathrm{D}_{40}}{\mathrm{D}_{25}}\right)+118.79\left(\frac{\mathrm{D}_{40}}{\mathrm{D}_{10}}\right) \\
& -78.35\left(\frac{\mathrm{D}_{60}}{\mathrm{D}_{10}}\right)
\end{aligned}
$$


16. The excellent predictive result by the proposed model is evident from the following regression equation for the samples tested in this study $\left(\mathrm{R}_{\mathrm{a}}^{2}=0.99\right)$ :

$$
Y=0.997 X-0.010
$$

when $\quad Y=$ the $\log$ of the calculated permeability

$X=$ the $\log$ of the measured permeability

17. Independent data have also been used to examine the proposed mode1. The following regression equation for the constant $v$ was obtained for the author's and Garcia-Bengochea's (1978)

$$
\begin{aligned}
& \operatorname{data}\left(R^{2}=0.89 \text { and } R_{a}^{2}=0.84\right) \text { : } \\
& \ln v=-3.06-2.59\left(\frac{D_{40}}{D_{25}}\right)+14.97\left(\frac{D_{40}}{D_{10}}\right) \\
& -7.26\left(\frac{\mathrm{D}_{60}}{\mathrm{D}_{10}}\right)+4.63\left(\frac{\mathrm{D}_{60}}{\mathrm{D}_{25}}\right)-2.71\left(\frac{\mathrm{D}_{25}}{\mathrm{D}_{10}}\right) \\
& -3.85\left(\frac{D_{60}}{D_{40}}\right)
\end{aligned}
$$

18. The applicability of the proposed model is evident from the following regression equation for the author's and GarciaBengochea's (1978) data $\left(R_{a}^{2}=0.98\right)$ :

$$
Y=0.99 X-0.03
$$

\subsection{RECOMMENDATIONS FOR FUTURE RESEARCH}

Future research is recommended in the following areas:

1. Prediction of permeability by the proposed model should 
be extended to: (a) other compacted conditions of soils similiar to the ones tested here, (b) other laboratory compacted soils and (c) field compacted soils.

2. An investigation of the sand fabric through variation in PSD caused by different compaction methods and/or different sands but at the same total porosity should be carried out. An attempt could then be made to relate PSD of sands to their liquefaction potential.

3. This study established procedures by which PSD can be masured in sand. These procedures can now be used to explain and predict shear and compressibility benavior of sands.

4. Techniques for determining PSD in coarse sands and fine gravels are needed to exploit this parameter in the prediction of behavior of (a) drainage filters and (b) highway subbases. 
REFERENCES 


\section{REFERENCES}

1. Ahmed, S. (1971), "Pore Size and Its Effect on the Behavior of a Compacted Clay", MSCE Thesis, Purdue University, West Lafayette, Indiana, June, pp. 200, Also JHRP Report No. 16, Sept.

2. Ahmed, S., Love11, C. W. Jr. and Diamond, S. (1974), "Pore Size and Strength of Compacted Clay", Journal of the Geotechnical Engineering Division, ASCE, Vol. 100, No. GT4, April, pp. 407-425.

3. Bhasin, R. N. (1975), "Pore Size Distribution of Compacted Soils After Critical Region Drying", Ph.D. Thesis, Purdue University, West Lafayette, Indiana, May, pP. 222, Also JHRP Report No. 75-3, March.

4. Black, D. K. and Lee, K. L. (1973), "Saturating Laboratory Samples by Back Pressure", Journal of the Soil Mechanics and Foundations Division, ASCE, Vol. 99, No. SM1, pp. 75-93.

5. Brewer, R. (1964), "Fabric and Mineral Analysis of Soils", J. Wiley and Sons, Inc., New York, Pp. 482

6. Carman, P. C. (1956), "Flow of Gases through Porous Media", Academic Press, Inc., New York, pp. 182 .

7. Cebeci, Z., Demirel, T. and Lohnes, R. A. (1978), "Evaluation of Hysteresis in Mercury Intrusion Porosimetry by Second-Intrusion Method", Research Record No. 675, TRB, pp. 15-20.

8. Childs, E. C. and Collis-George, N. (1950), "The Permeability of Porous Materials", Proceedings, Royal Society of London, Vol.
A201, pp. 392-405.

9. Csathy, T. D. and Townsend, D. L. (1962), "Pore Size and Field Frost Performance of Soils", Highway Research Board, Bulletin 331 , pp. 67-80.

10. Diamond, S. (1970), "Pore Size Distributions in Clays", Clays and Clay Minerals, Vol. 18, pp. 7-23. 
11. Diamond, S. (1971), "Microstructure and Pore Structure of ImpactCompacted Clays", Clays and Clay Minerals, Vo1. 19, pp. 239-249.

12. Diamond, S. and Dolch, W. L. (1972), "Generalized Log-Normal Distribution of Pore Sizes in Hydrated Cement Paste", Journal of Colloid and Interface Science, Vol. 34, No. 1; January, pp. 234-244.

13. Drake, L. C. and Ritter, H. L. (1945), "Macropore-Size Distributions in Some Typical Porous Substances", Industrial and Engineering Chemistry, Analytical Edition, Voi. 17, No. 12, December, pp. 787-791.

14. Ellison, A. H., Klem, R. B. and Schwartz, A. M. (1967), "Contact Angles of Mercury on Various Surfaces and the Effect of. Temperature", Journal of Chemical and Engineering Data, Vol. 12, No. 4, October, PP. 607-609.

15. Garcia-Bengochea, I. (1978), "The Relation Between Permeability and Pore Size Distribution of Compacted Clayey Silts", MSCE Thesis, Purdue University, May, pp. 179, Also JHRP Report No. 78-4, April

16. Garcia-Bengochea, I., Love11, C. W. and Altschaeff1, A. G. (1979), "Relation Between Pore Size Distribution and Permeability of Silty Clay", Journal of the Geotechnical Engineering Division, ASCE, Vol. 105, No. GT7, July, pp. 839-856.

17. Garcia-Bengochea, I., Lovel1, C. W. and Altschaeff1, A. G. (1980), "Closure to 'Pore Distribution and Permeability of Silty Clays"", Journal of the Geotechnical Engineering Division, ASCE, Vol. 106, No. GT10, October, pP. 1168-1170.

18. Gaskin, P. N. and Raymond, G. P. (1973), "Pore Size Distribution as a Frost Susceptibility Criterion", Proceedings of the Symposium on Frost Action on Roads, Norwegian Road Research Latoratory, Oslo, Vo1. 1, pp. 295-309.

19. Green, R. E. and Corey, J. C. (1971), "Calculation of Hydraulic Conductivity: A Further Evaluation of Predictive Methods", Proceedings, American Society of Soil Science, Vol. 35, pp. 3-8.

20. Hazen, A. (1911), "Discussion of "Dams on Sand Foundations"", Transactions, ASCE, Vol. 73, p. 199.

21. Hillel, D. (1980), "Fundamentals of Soil Physics", Published by Academic Press, Inc., New York, pp. 413.

22. Holtz, R. D. and Kovacs, W. D. (1981), "An Introduction to Geotechnical Engineering", Prentice-Hall, Inc., p. 106. 
23. Jackson, R. A. (1972), "On the Calculation of Hydraulic Conduct1vity", Proceedings, American Socicty of Soil Sclence, Vol. 36 , pp. $380-3 \overline{83}$.

24. Kemball, C. (1946), "On the Surface Tension of Mercury", Transactions, Faraday Society, Vol. 42, Pp. 526-537.

25. Kenney, T. C. (1980), "Frost-Heaving Rate Predicted from Pore-Size Distribution: Discussion", Canadian Geotechnical Journal, Vol. 17 , No. 2 , pp. 332-333.

26. Klock, G. O., Boersma, L. and DeBacker, L. W. (1969), "Pore Size Distributions as Measured by the Mercury Intrusion Method and Their Use in Predicting Permeability", Proceedings, American Society of Soil Science, Vol. 33, No. 1, PP. 12-15.

27. Kunze, R. J., Uehara, G. and Graham, K. (1968), "Factors Important in the Calculation of Hydraulic Conductivity", Proceedings, American Society of Soil Science, Vol. 32, Pp. 760-7 $\overline{65}$.

28. Lambe, T. W. (1951), "Soil Testing for Engineers", J. Wiley and Sons, Inc., New York, pp. 165.

29. Lambe, T.W. (1954), "The Permeability of Fine-Grained Soils", Permeability of Soils, ASTM STP 163, American Society of Testing Materials, Pp. 56-67.

30. Lohnes, R. A., Tuncer, E. R. and Demirel, T. (1976), "Pore Structures of Selected Hawaiian Soils", Research Record No. 512, TRB, PP. 76-69.

31. Lowel1, S. (1979), "Introduction to Powder Surface Area", Published by John Wiley \& Sons., Inc., New York, Pp. 199.

32. Mahmood, A. (1973), "Fabric-Mechanical Property Relationships in Fine Granular Materials", Clays and Clay Minerals, Vol. 22, No. 516, pP. 397-408.

33. Marshal1, T. J. (1958), "A Relation Between Permeability and Size Distribution of Pores", Journal of Soil Science, Vol. 9 , No. 1, pp. 1-8.

34. Meyer, H. I. (1953), "Pore Distribution in Porous Media", Journal of Applied Physics, Vol. 24, pp. 510-512.

35. Millington, R. J. and Quirk, J. P. (1959), "Permeability of Porous Media" Nature, Vol. 183, February, pp. 387-388. 
36. M1tchel1, J. K. (1956), "The Fabric of Natural Clays and Its Relation to Engineering Properties", Proceedings, Highway Research Record, Vol. 35, pp. 693-713.

37. Mitchel1, J. K., Hooper, D. R. and Campanella, R. G. (1965), "Permeability of Compacted Clay", Journal of the Soil Mechanics and Foundation Division, ASCE, Vol. 91, SM4, PP. 41-65.

38. Mitchell, J. K., Chatoian, J. M. and Carpenter, G. C. (1976), "The Influences of Sand Fabric on Liquefaction Behavior", Report No. TE 76-1, Dept. of Civil Engineering, University of California, Berkeley, June.

39. Murota, A. and Sota, K. (1969), "Statistical Determination of Permeability by the Pore Size Distribution in Porous Media", Proceedings, The 13th Congress of the International Association for Hydraulic Research, Japan, Vol. 4, September, pp. 183-190.

40. Oda, M. (1972), "Inftial Fabrics and Their Relations to Mechanical Properties of Granular Material", Soils and Foundations, JSCE, Vol. 12, No. 1, pP. 1-18.

41. Oda, M. (1976), "Fabrics and Their Effects on the Deformation Behaviors of Sand", Special Issue, Department of Foundation Engrg., Fac. of Engrg., Saitana University, Japan, pp. 59.

42. Orr, C.Jr. (1970), "Application of Mercury Penetration to Materials Analysis", Powder Technology, Vol. 3, No. 3, pp. 117-123.

43. Reed, M. A. (1977), "Frost Heaving Rate of Silty Soils as a Function of Pore Size Distribution", MSCE Thesis, Purdue University, August, pp. 116, Also JHRP Report No.77-15, September.

44. Reed, M. A., Love11, C. W., Altschaeffl, A. G. and Wood, L. E. (1979), "Frost-Heaving Rate Predicted from Pore Size Distribution", Canadian Geotechnical Journa1, Vol. 16, No. 3, August, pp. 463-472.

45. Reed, M. A., Lovell, C. W., Altschaeffl, A. G. and Wood, L. E. (1980), "Reply to Discussion on 'Frost-Heaving Rate Predicted from Pore Size Distribution"", Canadian Geotechnical Journal, Vol. 17, No. 4, November, pp. 639-640.

46. Rootare, H. M. (1968), "A Short Literature Review of Mercury Porosimetry as a Method of Measuring Pore Size Distribution in Porous Materials, and a Discussion of Possible Sources of Errors in This Method", Aminco Lab News, Vo1. 24, No. 3, Pp. 4A-4H. 
47. Scheidegger, A. E. (1957), "The Physics of Flow through Porous Media", University of Toronto Press, Pp. 351.

48. Scott, R. F. (1963), "Principles of Soil Mechanics", AddisonWesley Publishing Co., London, PP. 550 .

49. Taylor, D. W. (1948), "Fundamentals of Soil Mechanics", J. Wiley and Sons, Inc., New York, pp. 700.

50. Terzaghi, K. and Peck, R. B. (1948), "Soil Mechanics in Engineering Practice", J. Wiley and Sons, Inc., New York, Pp. 566.

51. Washburn, E. W. (1921), "Note on a Method of Determining the Distribution of Pore Sizes in a Porous Material", Proceedings, National Academy of Sciences, Vol. 7, pp. 115-116.

52. White, D. (1980), "The Fabric of a Medium Plastic Clay Compacted in the Laboratory and in the Field", MSCE Thesis, Purdue University, West Lafayette, Indiana, pp. 95, AIso JHRP Report No. 80-10, August.

53. Winslow, D. N. (1969), "The Pore Size Distribution of Portland Cement Paste", MSCE Thesis, Purdue University, West Lafayette, Indiana, January, pp. 102 .

54. Winslow, D. N. (1980), Personal Commication.

55. Winslow, D. N. and Love11, C. W. (1981), "Measurements of Porosity in Cements, Aggregates and Soils", accepted by Powder Technology, Special Issue on Mercury Porosimetry, Vo $\overline{1.29}$, No. 1.

56. Zoller, H. (1973), "Frost Heave and the Rapid Frost Heave Test", Public Roads, VoI. 37, No. 6, PP. 211-220. 


\section{APPENDICES}

Appendices not included in this copy of the report are:

Pages

Appendix A-1: Freeze Drying Method .......... $110-113$

Appendix A-2: Determination of Pore Size Distribution. - $114-127$

Appendix A-3: Computer Program for PSD Data

Reduction and plotting and

Sample Output. . . . . . . . . $128-137$

Append1x A-4: The Graphical Presentations of

PSD Origlnal Data........... 138

$\begin{aligned} \text { Appendix A-5: Use of the Computer Program } & 155 \\ & \text { SPLINE and Results . . . . . . . . . . . } 155\end{aligned}$

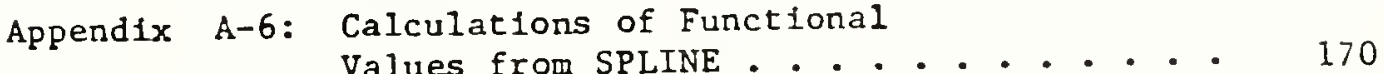

Appendix A-7: Correction of Unconnected Pores. . . . . 171

$\begin{aligned} \text { Appendix A-8: } & \begin{array}{l}\text { Pore Size Curves Expressed as a } \\ \text { Percentage of Total Pore Volume. . . . . }\end{array} 176\end{aligned}$

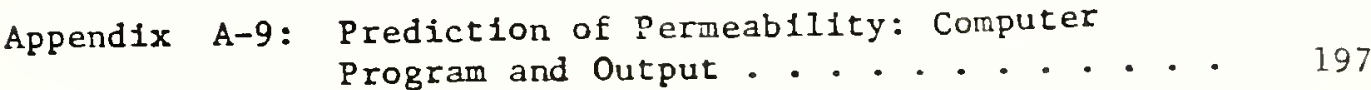

Appendix A-10: Prediction of Permeability :

Garcia-Bengochea's (1978) Data . . . . 225

A copy of each Appendix may be obtained at the cost of duplication

from:

Joint Highway Research Project

Civil Engineering Building

Purdue University

West Lafayette, Indiana 47907 
Purdue University Negative Numbers for Photographs

Figure

$3-4$

$3-6$

$3-6$ continued
Negative Number

$81196-3$

$81196-4$

$81196-9$

Appendix Figures
A-1-1
$75529-25$
$A-1-2$
$75529-28$
A-2-1
81196-12
A-2-2
81196-16
$A-2-3$
81196-17
A-2-5
80358-20
A-2-5 continued
81196-20 

$\bar{z}$
$\frac{2}{0}$
$x$
0
0
0
0
0
4
4
2
0
2
0
0
$w$
0
$\alpha$
$w$
0
0
0 